Prepared in cooperation with the Florida Fish and Wildlife Conservation Commission

\title{
Status and Threats Analysis for the Florida Manatee (Trichechus manatus latirostris), 2016
}

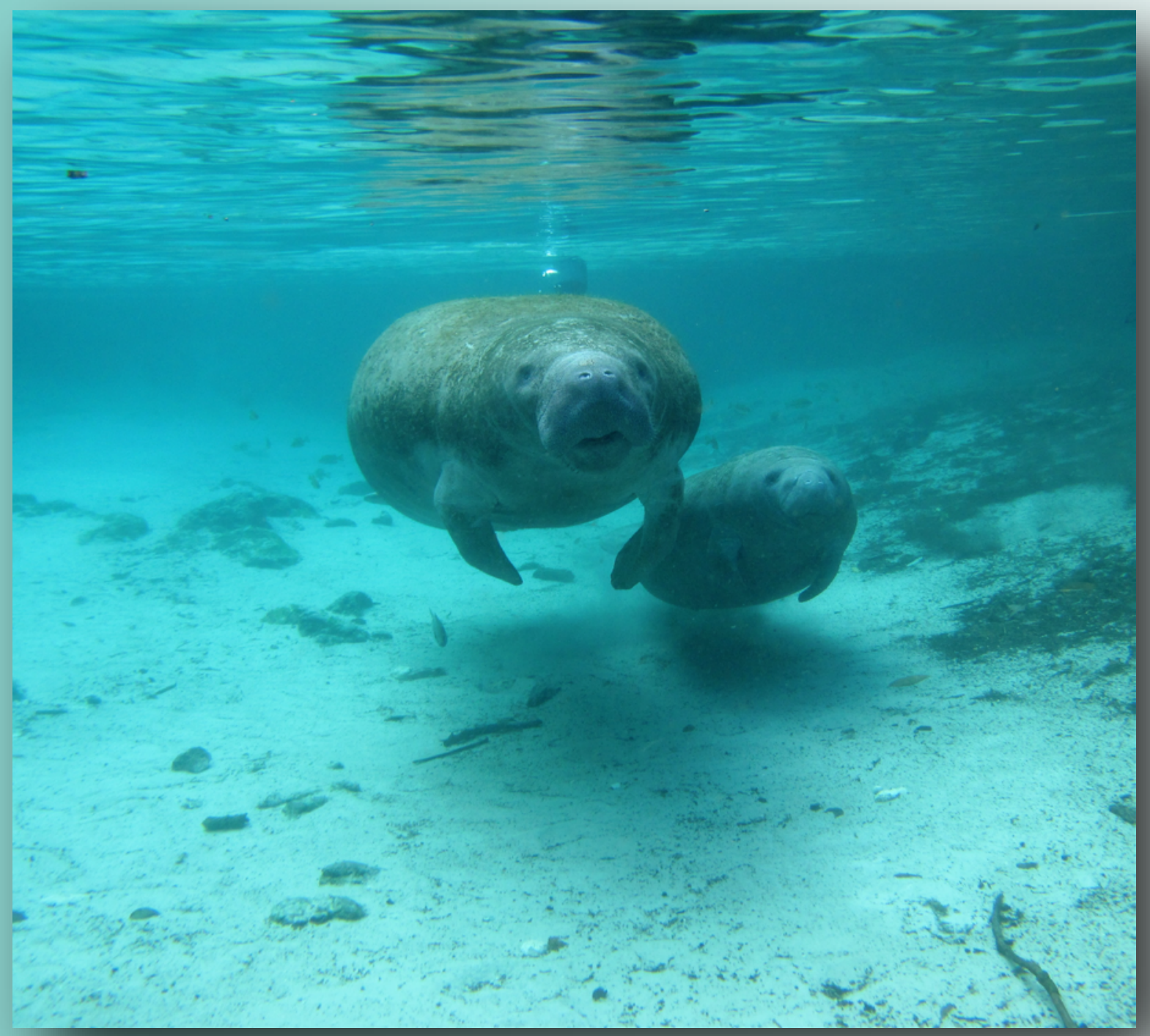

Scientific Investigations Report 2017-5030 
Cover: Photo of mother and calf Florida manatee at Crystal River National Wildlife Refuge, 26 January 2010. (Photograph provided by Robert Bonde, U.S. Geological Survey) 


\section{Status and Threats Analysis for the Florida Manatee (Trichechus manatus latirostris), 2016}

By Michael C. Runge, Carol A. Sanders-Reed, Catherine A. Langtimm, Jeffrey A. Hostetler, Julien Martin, Charles J. Deutsch, Leslie I. Ward-Geiger, and Gary L. Mahon

Prepared in cooperation with the Florida Fish and Wildlife Conservation Commission

Scientific Investigations Report 2017-5030 


\title{
U.S. Department of the Interior \\ RYAN K. ZINKE, Secretary
}

\section{U.S. Geological Survey William H. Werkheiser, Acting Director}

\author{
U.S. Geological Survey, Reston, Virginia: 2017
}

For more information on the USGS - the Federal source for science about the Earth, its natural and living resources, natural hazards, and the environment—visit http://www.usgs.gov or call 1-888-ASK-USGS.

For an overview of USGS information products, including maps, imagery, and publications, visit http://store.usgs.gov.

Any use of trade, firm, or product names is for descriptive purposes only and does not imply endorsement by the U.S. Government.

Although this information product, for the most part, is in the public domain, it also may contain copyrighted materials as noted in the text. Permission to reproduce copyrighted items must be secured from the copyright owner.

Suggested citation:

Runge, M.C., Sanders-Reed, C.A., Langtimm, C.A., Hostetler, J.A., Martin, Julien, Deutsch, C.J., Ward-Geiger, L.I., and Mahon, G.L., 2017, Status and threats analysis for the Florida manatee (Trichechus manatus latirostris), 2016: U.S. Geological Survey Scientific Investigation Report 2017-5030, 40 p., https://doi.org/10.3133/sir20175030.

ISSN 2328-0328 (online) 


\section{Acknowledgments}

The work described in this report would not have been possible without the extraordinary efforts of the field biologists, data managers, and analysts who have studied Florida manatees over the last three decades. We are particularly grateful to Cathy Beck, Howard Kochman, Gaia Meigs-Friend, Claudia Peñaloza, and Amy Teague of the U.S. Geological Survey; Martine de Wit, Holly Edwards, Andy Garrett, Jennifer Johnson, and Kari Rood of the Florida Fish and Wildlife Research Institute; and Sheri Barton of the Mote Marine Laboratory. This work has also benefitted from conversations with biologists who have generously shared their insights, among them, Nicole Adimey and Jim Valade of the U.S. Fish and Wildlife Service; Brandon Bassett, Alina Corcoran, Leanne Flewelling, Jan Landsberg, and Karen Steidinger of the Florida Fish and Wildlife Research Institute; Bob Bonde, Margaret Hunter, Bill Kendall, Jim Reid, and Dan Slone

of the U.S. Geological Survey; Dave Bruzek of Progress Energy; Jodie Gless of Florida Power and Light; Ron Mezich and Kent Smith of the Florida Fish and Wildlife Conservation Commission; Lori Morris of the St. Johns River Water Management District; Buddy Powell and Monica Ross of the Sea to Shore Alliance; Jane Provancha of the Kennedy Space Center; and John Reynolds of the Mote Marine Laboratory. Dawn Jennings and Jim Valade of the U.S. Fish and Wildlife Service have been instrumental in explaining the statutory and regulatory context for threats analysis under the Endangered Species Act of 1973. Chris Fonnesbeck of Vanderbilt University and Daniel Turek of Williams College provided substantive guidance on the fractions-of-mortality analysis. Lydia Stefanova of Florida State University helped develop the cold covariates. We are grateful to Tim Tinker, Jay Diffendorfer, and Ruth Larkins, who carefully reviewed this report and offered suggestions that have improved it substantially. 



\section{Contents}

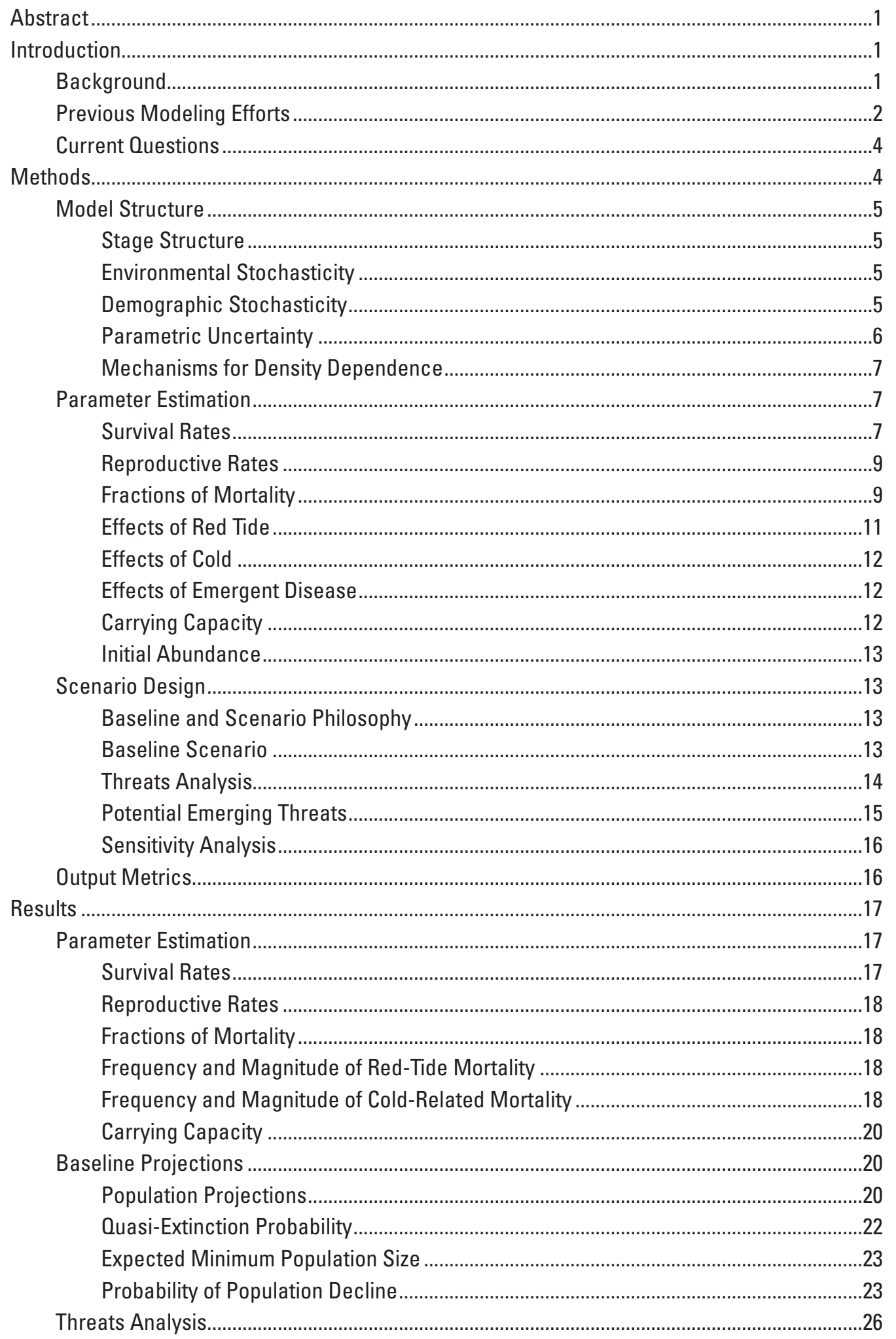




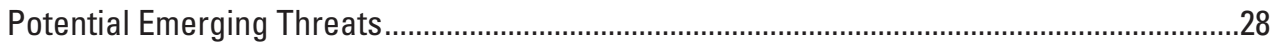

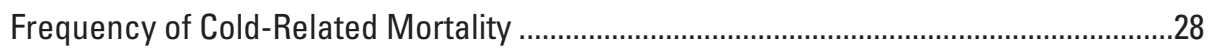

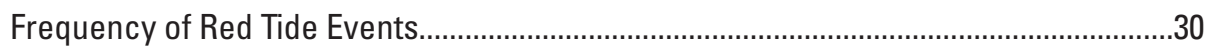

Chronic Atlantic Mortality ................................................................................................

Multiple Emerging Threats in Combination .......................................................................

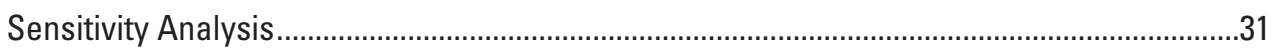

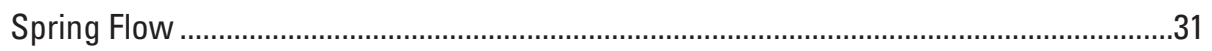

Behavioral Distribution Rules .........................................................................................33

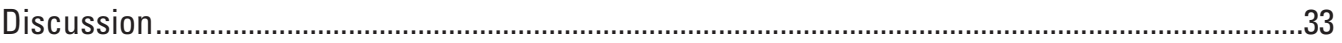

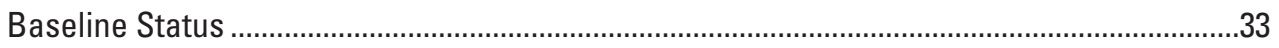

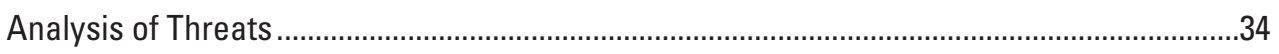

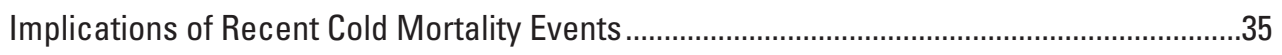

Implications of Recent Red-Tide Mortality Events ............................................................35

Public Comments on Previous Assessments .......................................................................35

Core Biological Model Developments.............................................................................

Summary and Conclusions....................................................................................................

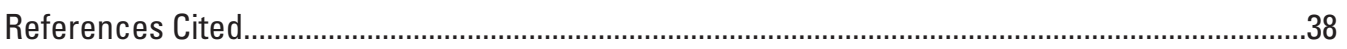

\section{Figures}

1. Diagram showing life-history stages and transitions for the manatee population model

2. Graphs showing models for Florida manatee carrying capacity as a function of time for four regions in Florida: $A$, Northwest; $B$, Upper St. Johns; $C$, Southwest; and $D$, Atlantic

3. Diagram showing directed acyclic graph of hierarchical model for estimating the fractions of mortality and additional mortality of Florida manatees associated with cold and red tide

4. Graphs showing baseline fractions of mortality of the Florida manatee, by cause of death and age class, in four regions of Florida, 1995-2013.

5. Graph showing estimated additional red-tide mortality rate for Florida manatees in the Southwest region of Florida, by intensity and age class, from the hierarchical analysis of manatee carcass data, 1995-2013

6. Graph showing estimated additional cold-related mortality rate for Florida manatees, by habitat quality, age class, and winter severity, in all regions of Florida combined, 1995-2013

7. Graphs showing estimated regional carrying capacity for Florida manatees in four regions of Florida, 2011-2110.

8. Graphs showing estimated Florida manatee population size under the baseline scenario, 2011-2110, for $A$, the total population size and $B$, the breeding adult population size in all four regions of Florida combined.

9. Graphs showing estimated Florida manatee breeding adult population size under the baseline scenario, 2011-2110, for $A$, the Gulf coast and $B$, the East coast of Florida. 
10. Graphs showing estimated Florida manatee breeding adult population size under the baseline scenario in four regions of Florida, 2011-2110.

11. Graphs showing probability of the adult population of Florida manatees falling below a quasi-extinction threshold within 50, 100, or 150 years from 2011, as a function of the quasi-extinction threshold under the baseline scenario on $A$, the Gulf coast, $B$, the East coast, or $C$, either coast of Florida

12. Histogram showing minimum total population size of Florida manatees on either the Gulf or East coast of Florida over the period 2011-2110.

13. Graph showing probability of the adult population of Florida manatees falling below a quasi-extinction threshold on either the Gulf or the East coast of Florida within 150 years from 2011 for the baseline scenario and five scenarios with the indicated threat removed.

14. Graph showing probability of the adult population of Florida manatees falling below 500 animals (quasi-extinction) on either the Gulf or the East coast of Florida within 100 years from 2011 as a function of watercraft-related mortality

15. Graph showing probability of the adult population of Florida manatees falling below 500 animals (quasi-extinction) on either the Gulf or East coast of Florida within 100 years from 2011 as a function of the level of loss of warm-water capacity with and without removal of the watercraft threat....

16. Graph showing probability of the adult population of Florida manatees falling below 500 animals on either the Gulf or the East coast of Florida within 150 years from 2011 as a function of the two primary threats, watercraft-related mortality and warm-water loss.

17. Histograms showing warm-water carrying capacity for Florida manatees in 2011, high- and medium-quality capacities combined across four regions of Florida, under two hypotheses for the 2009-11 cold-related mortality events: $A$, baseline hypothesis and $B$, density-dependent cold-related mortality hypothesis

18. Graphs showing probability of the adult population of Florida manatees falling below a quasi-extinction threshold within 150 years from 2011 as a function of the threshold under two hypotheses about the future frequency of red-tide mortality events in four regions of Florida. 


\section{Tables}

1. Baseline Florida manatee mean survival and reproductive rates for four regions in Florida, 1982-2015.

2. Estimated historical, 1996-2015, and forecast frequencies of Florida manatee red-tide mortality events in four regions of Florida for simulation years $0-40$.

3. Estimated magnitude of Florida manatee red-tide mortality events in two regions of Florida relative to mortality events in the Southwest region for simulation years $0-40$.

4. Probability of the adult Florida manatee population falling below a quasi-extinction threshold of 100, 250, or 500 animals on either the Gulf coast or the East coast of Florida in 50, 100, or 150 years from 2011 with threat removal scenarios

5. Probability of adult Florida manatee population declines of 10,30, or 50 percent at 100 years from 2011 compared to the 2011 adult population size, by state, coast, and region

6. Probability of the adult Florida manatee population on either the Gulf coast or the East coast of Florida falling below 100, 250, or 500 animals in 100 years from 2011, using the emerging threats scenarios

7. Sensitivity of probability of Florida manatee quasi-extinction and expected minimum population size to uncertainty in the long-term change in warm-water carrying capacity provided by springs on the Gulf coast or East coast of Florida

8. Sensitivity of probability of Florida manatee quasi-extinction and expected minimum population size to uncertainty about the behavioral rules used by manatees to distribute among warm-water sites on the Gulf coast or East coast of Florida. 


\section{Conversion Factors}

International System of Units to U.S. customary units

\begin{tabular}{lll}
\hline \multicolumn{1}{c}{ Divide } & By & To obtain \\
\hline & Length & \\
\hline centimeter $(\mathrm{cm})$ & 2.54 & inch (in.) \\
kilometer $(\mathrm{km})$ & 1.609 & mile (mi) \\
\hline & Volume & \\
\hline liter $(\mathrm{L})$ & 3.785 & gallon (gal) \\
\hline
\end{tabular}

Temperature in degrees Celsius $\left({ }^{\circ} \mathrm{C}\right)$ may be converted to degrees Fahrenheit $\left({ }^{\circ} \mathrm{F}\right)$ as follows: ${ }^{\circ} \mathrm{F}=\left(1.8 x^{\circ} \mathrm{C}\right)+32$.

\section{Abbreviations}

AT

Barker/RD

CBM

$\mathrm{Cl}$

$\mathrm{DOI}$

EMP

ESA

FWC

FWRI

FWS

IRL

IUCN

MIPS

MMPA

Mote

NW

PVA

SD

SE

SW

UME

USGS

USJ
Atlantic region

Barker closed-population robust design model

Core Biological Model

Credible interval

U.S. Department of the Interior

Expected minimum population size

Endangered Species Act of 1973, as amended (16 U.S.C. §1531 et seq.)

Florida Fish and Wildlife Conservation Commission

Florida Fish and Wildlife Research Institute

U.S. Fish \& Wildlife Service, Department of the Interior

Indian River Lagoon, Florida

International Union for Conservation of Nature

Manatee Individual Photo-identification System

Marine Mammal Protection Act

Mote Marine Laboratory

Northwest region

Population viability analysis

Standard deviation

Standard error of the mean

Southwest region

Unusual mortality event

U.S. Geological Survey

Upper St. Johns River region 



\title{
Status and Threats Analysis for the Florida Manatee (Trichechus manatus latirostris), 2016
}

\author{
By Michael C. Runge', Carol A. Sanders-Reed ${ }^{2}$, Catherine A. Langtimm', Jeffrey A. Hostetler ${ }^{3}$, \\ Julien Martin', Charles J. Deutsch ${ }^{3}$, Leslie I. Ward-Geiger ${ }^{3}$, and Gary L. Mahon ${ }^{1}$
}

\section{Abstract}

Trichechus manatus (West Indian manatee), especially T. m. latirostris, the Florida subspecies, has been the focus of conservation efforts and extensive research since its listing under the Endangered Species Act of 1973. To determine the status of, and severity of threats to, the Florida manatee, a comprehensive revision and update of the manatee Core Biological Model was completed and used to perform a population viability analysis for the Florida manatee. The probability of the Florida manatee population falling below 500 adults on either the Gulf or East coast within the next 100 years was estimated to be 0.42 percent. This risk of quasi-extinction is low because the estimated adult survival rates are high, the current population size is greater than 2,500 on each coast, and the estimated carrying capacity for manatees is much larger than the current abundance estimates in all four regions of Florida. Three threats contribute in roughly equal measures to the risk of quasi-extinction: watercraft-related mortality, red-tide mortality, and loss of warm-water habitat. Only an increase in watercraft-related mortality has the potential to substantially increase the risk of quasi-extinction at the statewide or coastal level. Expected losses of warm-water habitat are likely to cause a major change in the distribution of the population from the regions where manatees rely heavily on power plant effluents for warmth in winter (Southwest and Atlantic regions) to the regions where manatees primarily use natural springs in winter (Northwest and Upper St. Johns regions). The chances are nearly 50 percent that manatee populations in the Southwest and Atlantic regions will decrease from their 2011 levels by at least 30 percent over the next century.

A large number of scenarios were examined to explore the possible effects of potential emerging threats, and in most of them, the risk of quasi-extinction at the coastal scale within 100 years did not rise above 1 percent. The four exceptions are scenarios in which the rate of watercraft-related mortality increases, carrying capacity is only a fraction of the current estimates, a new chronic source of mortality emerges, or

\footnotetext{
${ }^{1}$ U.S. Geological Survey.
}

${ }^{2}$ Natural Systems Analysts, Inc.

${ }^{3}$ Florida Fish and Wildlife Conservation Commission. multiple threats emerge in concert. Even in these scenarios, however, the risk of falling below 500 adults on either the East coast or the Gulf coast within 100 years from 2011 is less than 10 percent. High adult survival provides the population with strong resilience to a variety of current and future threats. On the basis of these analyses, we conclude that if these threats continue to be managed effectively, manatees are likely to persist on both coasts of Florida and remain an integral part of the coastal Florida ecosystem through the 21 st century. If vigilance in management is reduced, however, the scenarios in which manatees could face risk of decline become more likely.

\section{Introduction}

Trichechus manatus Linnaeus (West Indian manatee) is a marine mammal endemic to the southeastern United States, the Greater and Lesser Antilles, the eastern coast of Mexico and Central America, and the northeastern coast of South America (Lefebvre and others, 2001). It was listed as endangered as part of the original enactment of the U.S. Endangered Species Act of 1973, as amended (ESA; 16 U.S.C. $\$ 1531$ et seq.). In 2016, the U.S. Fish and Wildlife Service (FWS) proposed reclassification of the West Indian manatee from endangered to threatened (U.S. Fish and Wildlife Service, 2016). To provide information during the preparation of the proposed rule, an interim status and threats assessment for manatees in Florida was published (Runge and others, 2015); this report augments and completes that analysis.

\section{Background}

Manatees are long-lived, large mammals in the Order Sirenia (Family Trichechidae) found in shallow tropical and subtropical coastal waters and freshwater rivers of three continents, including North America, South America, and Africa. The West Indian manatee consists of two subspecies: Trichechus manatus latirostris (Florida manatee), which occurs in coastal and freshwater systems of the southeastern United States, and Trichechus manatus manatus (Antillean manatee), which except for a population in Puerto Rico, occurs outside the United States (Lefebvre and others, 2001). This report 
focuses on the status of Florida manatees.

The primary threats to Florida manatees, as identified in the most recent recovery plan, are collisions with watercraft, potential loss of warm-water refuges, and coastal development (U.S. Fish and Wildlife Service, 2001). Since the early 1970s, however, the population of Florida manatees appears to have grown considerably. A 2011-12 survey estimated the abundance to be 6,350 animals (95\% confidence interval, 5,310-7,390) (Martin and others, 2015). In 2007, a status and threats assessment was conducted which confirmed that watercraft-related mortality and loss of warm-water refuges represent the largest threats to manatee persistence; the assessment also provided a quantitative estimate of the risk of extinction (Runge and others, 2007b). This assessment was integrated into the 5-year status review conducted by U.S. Fish and Wildlife Service (FWS; 2007) in which FWS recommended downlisting the species to threatened.

Since 2007, substantial improvements to the monitoring and analysis of Florida manatee life-history parameters have been achieved (Langtimm, 2009; Kendall and others, 2012; Kendall and others, 2013; Peñaloza and others, 2014; Langtimm and others, 2016). New assessments of manatee carrying capacity have been conducted (Provancha and others, 2012; M.C. Runge, USGS and C.J. Deutsch, Florida Fish and Wildlife Research Institute [FWRI], written commun., 2017), and a new aerial survey method for estimating abundance has been developed (Martin and others, 2015). At the same time, a cluster of puzzling events has occurred: severe cold-related mortality of Florida manatees in the winters of 2009-10 and 2010-11, an intense red-tide mortality event in the Southwest region in 2013, and a mortality event of unknown cause that began in Brevard County in 2012. In addition, during 201016 , three power plants on the Atlantic coast that provide warm water to manatees have been demolished and rebuilt.

The basis of status assessment under the ESA is an evaluation of the five factors identified in section 4(a)(1) of the statute (habitat loss, overutilization, disease or predation, inadequacy of existing regulatory mechanisms, and other natural or manmade factors). As noted by Runge and others (2007b), one of the challenges in a five-factor analysis is understanding how the threats interact. In this report, we continue the practice of using an overarching quantitative definition of risk - the probability of quasi-extinction (Morris and Doak, 2002) - as a measure of status that reflects the statutory language in the ESA (Runge and others, 2007b; Runge and others, 2015). Using the probability of quasi-extinction as a metric can integrate the threats identified by the five factors, quantify their relative effects, and provide an understanding of the trade-offs among them. To estimate the probability of quasi-extinction, a population model was needed that is designed to forecast the dynamics of the population, taking into account the species' life history, available demographic information, forecasts of threats and how they affect vital rates and carrying capacity, environmental variability, and uncertainty about the dynamics and parameter estimates.

\section{Previous Modeling Efforts}

There has been an evolution of methods to forecast population dynamics for West Indian manatees. In this report, the focus is on the development of the methods for describing the Florida manatee population, which has been studied extensively. Only one effort has been undertaken to predict the viability of the Antillean manatee across its range (Castelblanco-Martínez and others, 2012).

Three early efforts were made to model the Florida manatee population. Packard (1985) developed a deterministic, age-structured model of manatee dynamics to estimate the population growth rate and analyze its uncertainty. Eberhardt and O'Shea (1995) undertook a similar effort with updated information; their model was used in the development of the quantitative recovery criteria in the Manatee Recovery Plan (U.S. Fish and Wildlife Service, 2001). Both of these efforts were deterministic and essentially retrospective in that they relied on life-history parameters estimated over the previous decades and did not make a specific attempt to forecast the future. Marmontel and others (1997) developed the first predictive population viability analysis (PVA) for Florida manatees, relying on available software (VORTEX) to build a stochastic, age-structured model. There was a methodological error, however, in the way in which Marmontel and others (1997) estimated survival rates, which rendered their forecasts unreliable (Runge and others, 2004).

In 2002, the research arm of the Florida Fish and Wildlife Conservation Commission (FWC) developed a PVA, again using VORTEX (Florida Marine Research Institute, 2002). This model included age structure, environmental and demographic stochasticity, three types of catastrophes (virulent disease, red-tide mortality, cold), and density-dependent reproductive rates. The model treated the population in Florida as a metapopulation consisting of the four regions that FWS and FWC use as management units (Northwest [NW], Upper St. Johns River [USJ], Atlantic [AT], and Southwest [SW]). The regions were connected by dispersal rates, and there was positive correlation in the environmental variation among them. The initial population size was established from the 2001 synoptic aerial survey. Survival rates were estimated from mark-recapture analysis of data from the Manatee Individual Photo-identification System (MIPS). The model employed estimates of carrying capacity that were simply set at twice the initial population size. The model did not include parametric uncertainty. Sixteen scenarios were considered under a combination of hypotheses about whether survival rates and carrying capacity would stay stable or decrease. This population model had many of the components that were desired, but the constraints of using a modeling package prevented all of the desired elements from being included.

The basis of present-day modeling for Florida manatees began with a stage-structured, deterministic model (Runge and others, 2004) informed by mark-recapture estimates of survival (Langtimm and others, 2004) and reproduction (Kendall 
and others, 2004) for the four regions. The stage structure, rather than age structure, allowed us to better capture the lifehistory dynamics of female manatees. Although the purpose of this model was to estimate historical population growth rates in the four regions, it was structured to allow development into a stochastic, forecasting model.

In 2003, FWS undertook an assessment under the Marine Mammal Protection Act (MMPA) to evaluate the effect of take $^{1}$ from watercraft activities on Florida manatees. As part of this rulemaking, a population model was developed that extended the structure of Runge and others (2004) to allow forecasting and the evaluation of incidental lethal take (Runge, 2003) $)^{2}$. The incidental-take model combined advances from Runge and others (2004) and Florida Marine Research Institute (2002) and augmented them. This model separately tracked females and males and was the first manatee PVA model to include parametric uncertainty. It included two types of catastrophic stochasticity (virulent disease and red-tide mortality); the third type (cold), used in an earlier model, was coupled to the distributions that represented environmental stochasticity. Like Florida's 2002 PVA, this model used the 2001 synoptic counts as the initial population size and kept track of the four regions separately. Carrying capacity in each of the four regions, currently and forecast over time, was estimated by an expert panel in 2002 . Two mechanisms were used for density dependence: a reduction in reproductive rates as a population increased toward its carrying capacity and additional mortality in cold years when the population size exceeded the carrying capacity. Finally, this model introduced the concept of the fraction of mortality owing to a particular threat (watercraft-related mortality) and estimated it using data from the State's carcass salvage and necropsy program.

In 2006, the U.S. Geological Survey (USGS) and FWC scientists worked together to develop the Core Biological Model (CBM). The idea was to have a common core modeling framework for Florida manatees that could be used by multiple agencies for a variety of assessment purposes. The 2006 version of the Core Biological Model is now referred to as "CBM version 2" (CBMv2); it was a direct extension of the incidental take model of Runge (2003), which is now referred to as "CBMv1." The second version of the CBM was first used to evaluate Florida manatees with respect to Florida's imperiled species classification criteria (Haubold and others, 2006). Soon after, the findings were applied to assign an updated International Union for Conservation of Nature (IUCN) Red List category to T. manatus and its Florida subspecies (Deutsch and others, 2008). The core structure and parameters of CBMv1 and CBMv2 were mostly the same. The differences were in technical aspects of the coding; CBMv2 introduced parallelism across scenarios, which increased the power to

\footnotetext{
${ }^{1}$ Under the MMPA, "take" means "to harass, hunt, capture, kill, or attemp to harass, hunt, capture, or kill any marine mammal” (16 USC §1362(13)).

${ }^{2}$ Note that development of the deterministic model of Runge and others (2004) preceded development of the predictive model of Runge (2003); the publication of the two works was reversed in time.
}

describe the effects of factors being tested. Also, CBMv1 and CBMv2 differed in which fractions of mortality they could accommodate because the methods for analyzing fractions of mortality were still in development.

In 2007, FWS conducted a 5-year review of the status of the West Indian manatee, as required under section 4(c)(2)(A) of the ESA (U.S. Fish and Wildlife Service, 2007). To inform this evaluation, a new version of the CBM was developed and fully documented, CBMv3 (Runge and others, 2007a). The core structure and parameters in $\mathrm{CBMv} 3$ were the same as in $\mathrm{CBMv} 2$. The new features produced the ability to run a much wider range of scenarios, including the flexibility to adjust the fraction of mortality associated with watercraft, warm-water loss, red tide, water-control structures, and entanglement. New Bayesian methods were developed to analyze the carcass necropsy data and estimate the fractions of mortality associated with various causes of death. A comparative threats analysis was undertaken with CBMv3 to investigate the individual and combined effects of various threats on quasi-extinction (Runge and others, 2007b).

In 2009, USGS scientists developed a new version of the $\mathrm{CBM}(\mathrm{CBMv} 4)$ designed to estimate the optimum sustainable population level for Florida manatees, a quantity relevant to assessment under the Marine Mammal Protection Act. Again, the core structure and parameters remained unchanged from $\mathrm{CBMv} 3$, but additional capability for systematically evaluating the effects of anthropogenic take was added. As of 2017, work on this application has not been finished or published.

In 2011, FWS began the process of a new 5-year review, and USGS and FWC scientists worked together to update the CBM (CBMv5). This involved substantial changes to the structure of the model, as well as a comprehensive review of all parameters (Runge and others, 2015). The structural changes included recognition of three types of years with respect to winter severity (normal, cold, severely cold); classification of warm-water carrying capacity into four types (high-quality, medium-quality natural, medium-quality industrial, and low-quality); expansion of the density-dependent mechanism associated with warm-water limitation to separate the effects on calves, subadults, and adults; development of two alternative behavioral rules for distribution of manatees among warm-water sites (thermal quality selection, ecological trap); and more flexibility in the treatment of red-tide mortality events. Nearly all of the parameters in the model were updated with data that were available through the end of 2012. A new mark-recapture model that reduced bias and increased precision was used to estimate adult survival rate (Kendall and others, 2013; Langtimm and others, 2016), and a new, formal expert elicitation process for estimating warm-water carrying capacity was undertaken during 2011-12 (M.C. Runge, USGS and C.J. Deutsch, FWRI, written commun., 2017). The preliminary results of this interim modeling effort were provided to FWS in January 2013, but work on the 5-year review and publication of the results were halted because of spending cuts stipulated by the Budget Control Act of 2011 (Publ. L.112-25). These results were eventually published in 2015 (Runge and others, 2015). 
Status and Threats Analysis for the Florida Manatee (Trichechus manatus latirostris), 2016

\section{Current Questions}

At the time when results from CBMv5 were generated (late in 2012), there were some emerging questions about recent manatee mortality events in Florida. Severe cold-mortality events occurred in the winters of 2009-10 and 2010-11 (Barlas and others, 2011). Extensive loss of seagrass habitat occurred in the Indian River Lagoon in Brevard County, Fla., (AT region) in 2011 and 2012 (Phlips and others, 2015); a manatee unusual mortality event (UME), possibly associated with the seagrass loss and subsequent dietary change, peaked in this same area in 2013. Also in 2013, in the SW region, there was an unprecedented level of manatee mortality caused by red tide. None of these events were easily explained by previous data or model scenarios, and scientists and resource managers were thus unable to determine their relevance for the current and future status of Florida manatees. During 2012-13, a means of answering these questions was not available using the CBM because (1) the events were so recent that their effects had not yet appeared in the monitoring data and (2) the structure of the CBM did not allow formal evaluation of the questions that were being asked. The version of the CBM described in this report (CBMv6) was developed to incorporate the most recent information (data available as of September 2016) and to add additional capability to investigate the potential effects of what may be emerging threats.

With regard to the cold-related mortality events of 2009-10 and 2010-11, the importance of these events depends on the underlying causal mechanisms. We propose four hypotheses. (1) Those two winters were just back-to-back cold or severely cold years affecting a population that is larger than it once was. Thus, a large number of animals died because the population was larger; this represents normal variation in mortality that is already captured in the CBM. (2) Those two winters represent density-dependent cold-related mortality of the kind posited in the CBM when the population reaches or exceeds carrying capacity during cold years. This would indicate that the carrying capacity was approached or exceeded.

(3) Those two winters were out-of-the-ordinary severely cold events, inducing density-independent mortality because they caught animals away from their preferred warm-water habitat, and the ramifications were felt through a reduction in population size. (In the CBM, this hypothesis differs from hypothesis 1 in that it forces all animals to be exposed to severe cold in the first 2 years of the simulation). (4) Those two winters were harbingers of much more frequent severe cold in the future. This hypothesis is similar to hypothesis 1 as an explanation for those two mortality events but differs in terms of the expectations for future events. We developed scenarios in the CBM to compare these four hypotheses.

With regard to the red-tide mortality event in the SW in 2013, two questions arise. Are we observing a separate class of red-tide mortality (an "intense" red tide year) or was 2013 within the range of previous red-tide mortality years? Is the frequency of red-tide mortality increasing? From these questions, we developed four hypotheses and four corresponding scenarios to compare them. (1) There are only two classes of years with regard to red-tide mortality (background-level years, red-tide years), and the frequency in the future will be the same as in the recent past. (2) There are three classes of years (background, moderate red tide, intense red tide), but the future frequency will be the same as in the past. (3) There are two classes of years, but the frequency of red-tide mortality will be elevated in the future. (4) There are three classes of years, and the frequency of moderate and intense red-tide mortality will increase in the future.

The events taking place in the Indian River Lagoon (IRL) raise questions concerning the mechanisms by which manatees have been affected and might be affected in the future. One hypothesis is that the loss of seagrass brought about by local environmental changes (Phlips and others, 2015) has reduced the availability of this primary food for manatees. Manatees subsequently shifted their diet and have succumbed to mortality causes currently under investigation, but surviving animals will avoid this area in the future (thus producing a short-lived mortality event and a long-term reduction in regional carrying capacity). An alternate hypothesis is that the IRL event represents a density- and stage-independent mortality event, but the signals attracting manatees to the IRL remain, producing a long-term ecological trap that could result in chronic annual mortality in the future. Other hypotheses are possible; we focused on evaluating the chronic mortality hypothesis as a worst-case explanation.

In addition to addressing these particular questions that have arisen from recent mortality events, we have undertaken a full quantitative threats analysis, as we have in the past (Runge and others, 2007b; Runge and others, 2015), including investigation of a number of scenarios concerning watercraftrelated mortality, rate of loss of warm-water capacity provided by spring flows, and persistence of industrial warm-water effluents.

\section{Methods}

The basis of the assessment in this report is a comparative population viability analysis. This involves forecasting the Florida manatee population and evaluating the risk of quasi-extinction under scenarios that differ in the presence and level of various threats while accounting for process variation (environmental, catastrophic, and demographic stochasticity), as well as parametric and structural uncertainty. The model structure and the methods for estimating the parameters are mostly unchanged from earlier versions of the CBM (Runge and others, 2007a; Runge and others, 2015); the largest changes are in the flexibility to run a large range of scenarios. The CBM is written and executed in the MATLAB programming environment (MathWorks, Inc., version R2012b, https:// www.mathworks.com/). 


\section{Model Structure}

The manatee Core Biological Model version 6 (CBMv6) is a stage-based projection model for Florida manatees, incorporating stochasticity, density dependence, long-term change in carrying capacity, and parametric uncertainty. The model tracks manatees in the four geographic regions of Florida (NW, USJ, AT, and SW) separately and does not account for movement between them. (Although dispersal is known to occur among the regions, the rates of dispersal are low [Reid and others, 1991; Deutsch and others, 2003]). The overarching aspects of the structure of the model are described here; for mathematical details, see Runge and others (2007a). New features in CBMv6 are described in detail in later sections of this report.

Previous versions of the CBM used an annual cycle based on the calendar year (January 1 to December 31). In the current version, the CBM was restructured to an annual year beginning at the start of the winter monitoring period (December 1 to November 30). This pre-winter, rather than mid-winter, break between years was motivated by the new survival rate methods that include the use of auxiliary sightings (Kendall and others, 2013), but it also more naturally encompasses the seasonal threats experienced by manatees and thus provides a better structure to estimate survival (see "Survival Rates" in the "Methods" section) and mortality (see "Fractions of Mortality" in the "Methods" section).

\section{Stage Structure}

The CBM classifies and tracks manatees in 10 life-history stages. Immature animals (calves and subadults) are classified by age and sex, adult females are classified by reproductive conditions, and adult males are pooled into a single class (fig. 1). The transitions between stages are governed by survival $\left(s_{i}\right)$ and reproductive $\left(\gamma_{i}\right)$ parameters. The rationale and details of the stage classes are described by Runge and others (2007a). Note that first-year calves ( 0.5 years old) are not tracked separately in the model because they are considered a unit with the mother; animals appear in the model as secondyear calves ( 1.5 years old).

\section{Environmental Stochasticity}

The conditions in the near shore environment in Florida show annual variation, which results in annual variation in the survival and reproductive rates of manatees (Langtimm and others, 2004). In the CBM, three types of annual variation are included: normal annual variation in survival and reproductive rates, rare catastrophic events, and special events (red tide, cold).

Normal annual variation is modeled with temporal variation in the survival and reproductive rates, sampled from logit-normal probability distributions. All of the survival rates within a region are assumed to vary together with perfect correlation (so that a bad year for calves is also a bad year for adults, albeit with different variances). All of the breeding rates within a region are assumed to vary together with perfect correlation. The sets of survival and breeding rates are not assumed to vary together, but a correlation between them can be specified. Environmental variation in survival rates and reproductive rate among regions is assumed to be uncorrelated.

Catastrophes occur randomly with a given frequency (each year is treated as a Bernoulli trial); when they occur, they reduce the survival and reproductive rates proportionally by a substantial magnitude. The occurrence of catastrophes is not correlated with the normal annual variation.

The occurrence of red tide is treated as a generalized Bernoulli distribution with three possible classes (background, moderate, intense), each with its own frequency and magnitude of manatee mortality. In addition, in a red-tide year (moderate or intense), a multiplicative reduction in the reproductive rates occurs. The CBM allows for the occurrence of red tide to be correlated among the three regions in which it occurs (NW, AT, $\mathrm{SW})$; this is implemented by generating a three-dimensional Gaussian copula with the desired linear correlation matrix. The three marginal distributions of the copula are uniform $(0,1)$ and are used with the red-tide frequencies to determine the type of year in each region.

Likewise, the classification of years with regard to cold is also treated as a generalized Bernoulli distribution with three possible classes (normal, cold, severely cold), each with its own frequency. Cold and severely cold years produce densitydependent and density-independent effects on mortality, which are described in more detail in the section on "Mechanisms for Density Dependence." The CBM allows the occurrence of cold years to be correlated among the four regions; this is implemented by generating a four-dimensional Gaussian copula with the desired linear correlation matrix. The four marginal distributions of the copula are uniform $(0,1)$ and are used with the cold-year frequencies to determine the type of year in each region. Red tide and cold are not correlated with each other, neither within a region across years nor across regions.

\section{Demographic Stochasticity}

Demographic stochasticity is the variation that is generated by chance events that occur for individual animals (Lande, 1993). For example, the number of third-year males that survive in a given year is drawn from a binomial distribution with success probability equal to the year-specific survival rate for third-year males. The effect of demographic stochasticity is most pronounced for small population sizes. In the CBM, demographic stochasticity is applied to all the life-history transitions (survival events, reproductive events, and the sex of each calf), using binomial distributions, independently for each class in the model. In addition, any time the model requires animals to be distributed among stages, classes, or habitats, a multinomial or multivariate hypergeometric distribution is used; the added variance that results is a form of demographic stochasticity. 


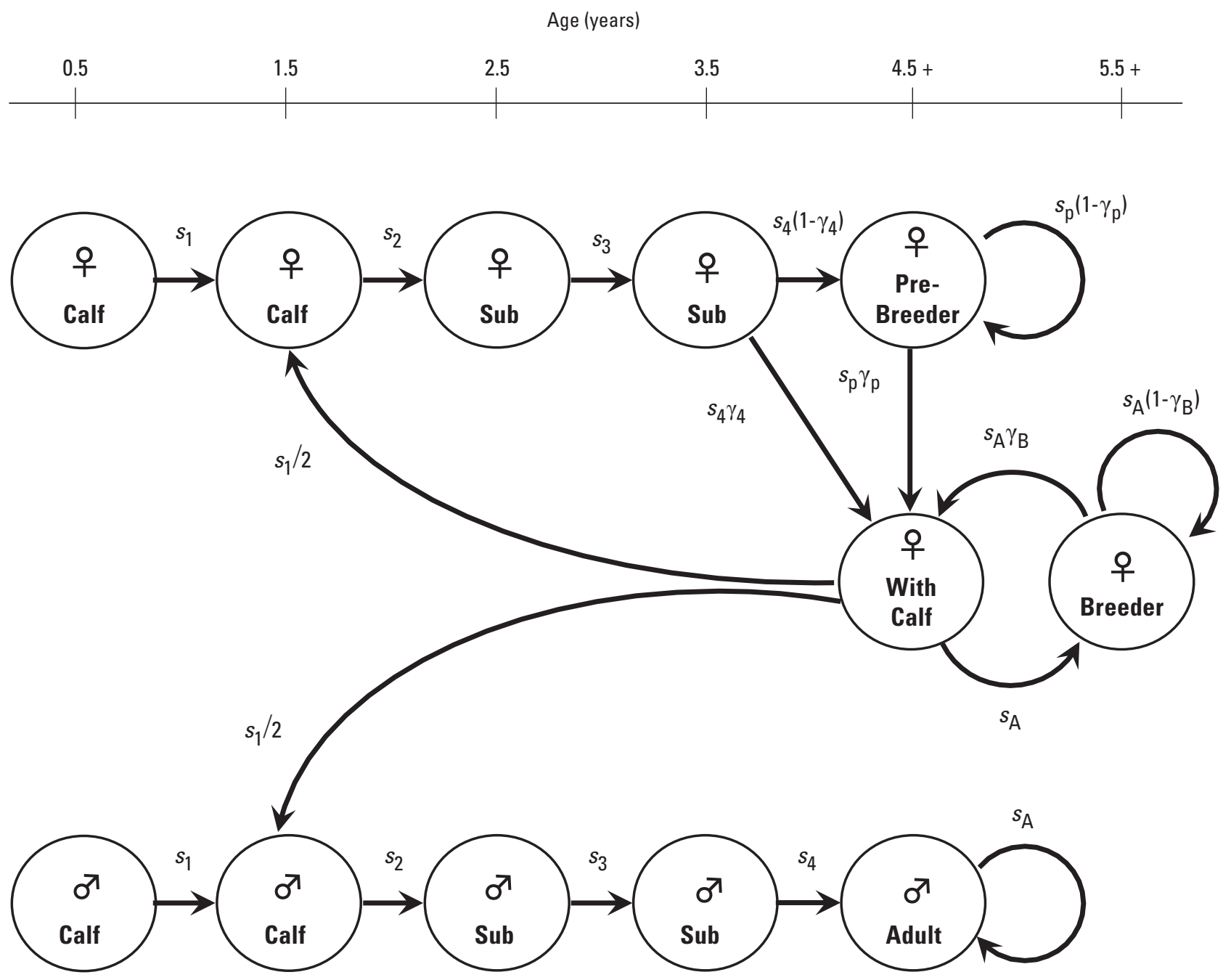

Figure 1. Life-history stages and transitions for the manatee population model. Sex- and stage-specific survival $(s)$ and reproductive $(\gamma)$ rates govern the transition between stages. Females $(+)$ and males $\left({ }^{\lambda}\right)$ are separately accounted for in the model. Calves enter the population model as separate entities at 1.5 years; until that time they are counted as part of the female-with-calf pair. (The 0.5 -year stage is shown for completeness but is not tracked separately in the model)

\section{Parametric Uncertainty}

An important feature of the CBM since its inception has been the distinction between parametric uncertainty and environmental variation (McGowan and others, 2011), and the appropriate inclusion of both. One of the major limitations of the PVA software packages is the inability to properly include parametric uncertainty; this was one of the motivations for creating the original CBM in a custom programming environment. Nearly every parameter in the CBM is sampled from a probability distribution that represents uncertainty in our knowledge. This sampling is done at the beginning of each replicate but not for each year within the replicate, so that each replicate simulation represents a possible combination of parameters describing manatee dynamics. In most cases, the probability distributions used to describe uncertainty are the natural distributions for the parameter in question. For example, uncertainty in survival rates and reproductive rates is modeled with a logit-normal distribution, which confines the rates to the interval $(0,1)$. Uncertainty in the temporal variance of survival rates is modeled with a chi-square distribution, and the frequencies of the three classes of red-tide years are sampled from a Dirichlet distribution (the multivariate analog of the beta distribution). Details can be found in Runge and others (2007a). One important addition to CBMv6 is the treatment of the initial population size as an uncertain quantity, which is sampled from a log-normal distribution for the NW, $\mathrm{AT}$, and $\mathrm{SW}$ regions and a uniform distribution for the USJ region. 


\section{Mechanisms for Density Dependence}

Florida manatees are found at the northern end of the range of West Indian manatees, and winter temperatures in Florida frequently approach levels that cause physiological stress (Bossart and others, 2002). Thus, one of the major limiting factors for manatee population growth is presumed to be the availability of warm-water refugia with food available nearby during the winter months (U.S. Fish and Wildlife Service, 2001; Laist and Reynolds, 2005b). The CBM contains two mechanisms for generating density dependence in lifehistory parameters; both are conditional on winter carrying capacity. First, the reproductive rates decrease as the post-winter population size (after removal of animals owing to coldrelated mortality) approaches the carrying capacity, where here carrying capacity is the sum of the capacities associated with high-quality natural, medium-quality natural, and mediumquality industrial warm-water habitats. The density-dependent reproductive rate is given by

where

$$
\gamma(N)=\gamma_{0}\left(1-\alpha\left(\frac{N}{K}\right)^{\beta}\right)
$$

$N \quad$ is the post-winter population size in a region,

$K$ is the sum of the warm-water capacities for that region,

$\alpha \quad$ is the fraction by which reproduction is reduced when the population is at its carrying capacity,

$\beta \quad$ controls how close the population size has to be to $K$ before the density-dependent effects are felt, and

$\gamma_{0} \quad$ is the reproductive rate at low population density.

The second mechanism for density dependence involves 27 differential mortality rates for calves, subadults, or adults in high-, medium-, or low-quality warm-water habitats, during normal, cold, or severely cold years. The mortality rates increase as animals are forced from high- to medium- to low-quality habitat and as the severity of the winter increases. CBMv6 provides three alternative behavioral rules to describe how manatees distribute among high-, medium-, and lowquality warm-water habitats as population size or carrying capacity changes: (1) the thermal quality selection rule ${ }^{3}$ (similar to the site pre-emption rule of Rodenhouse and others, 1997), in which high-quality sites fill first, then medium-quality sites (both natural and industrial), and finally low-quality sites; (2) an ecological trap rule (Schlaepfer and others, 2002), in which industrial sites fill first, then high-quality natural sites, then medium-quality natural sites, and finally lowquality sites; and (3) a historical distribution rule, in which

\footnotetext{
${ }^{3}$ This was referred to incorrectly as the "ideal despotic rule" by Runge and others (2015).
}

manatees fill the types of warm-water habitat in proportion to historical distribution among those types.

Governing both of these mechanisms of density dependence are estimates of regional carrying capacity, where carrying capacity is understood to be the number of manatees that can find suitable winter habitat, taking into account the thermal characteristics of the sites, the availability of food nearby, and the behavior of manatees. During 2011-12, an expert panel was convened to estimate the current regional carrying capacities for manatees and to forecast those carrying capacities over the next 100 years (M.C. Runge, USGS and C.J. Deutsch, FWRI, written commun., 2017). Before providing estimates of carrying capacity, the panel discussed how to structure the warm-water capacity model. First, they expressed a desire to distinguish four types of warm-water habitat:

(1) high-quality sites, which include first-order natural springs; (2) medium-quality natural sites, which include smaller springs and other sources of warm water, such as passive thermal basins (Laist and Reynolds, 2005a); (3) medium-quality industrial sites, which are produced by warm-water effluent from power-generating stations; and (4) low-quality sites, which attract manatees at some times during the winter but which do not provide enough warmth for manatees to survive cold spells. Second, for each region, the panel sketched out the shape of the function that best represented what they thought would occur over time to the capacities associated with highand medium-quality habitats (fig. 2). For example, in the NW region, there is an expectation that warm-water capacity at springs (both high- and medium-quality habitat) will decline exponentially over time owing to reduction in spring flow, and industrial warm-water habitat will disappear abruptly at some point in the future (there is only one power-generating facility in the NW). Third, a formal process of elicitation was undertaken to estimate the parameters for the models depicted in figure 2, taking account of uncertainty (see "Carrying Capacity" in the "Methods" section).

\section{Parameter Estimation}

As noted previously, in this update to the CBM, nearly all of the parameter estimates were revisited because new data were available, new methods of analysis had been developed, or new insights were needed from experts. All the information available as of September 2016 was used.

\section{Survival Rates}

Adult survival rates were estimated from mark-resighting data in the interagency MIPS database using the Barker closed-population robust design (Barker/RD; Kendall and others, 2013) run in the program MARK (White and Burnham, 1999). For NW, USJ, AT, data processing was complete through the 2014 sampling season (winter 2013-14); for the SW region, data processing was complete through the 2015 sampling season. Two covariates (red tide, cold) were tested 


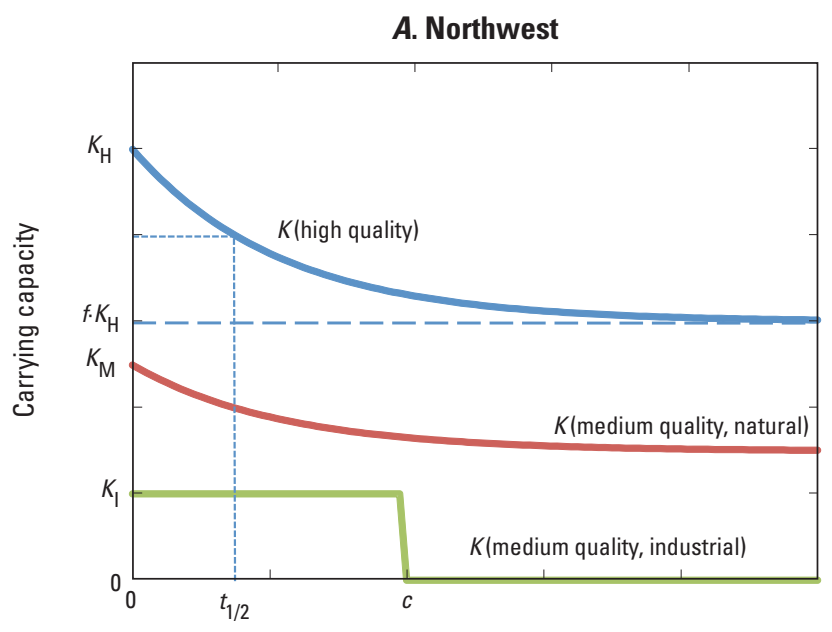

C. Southwest

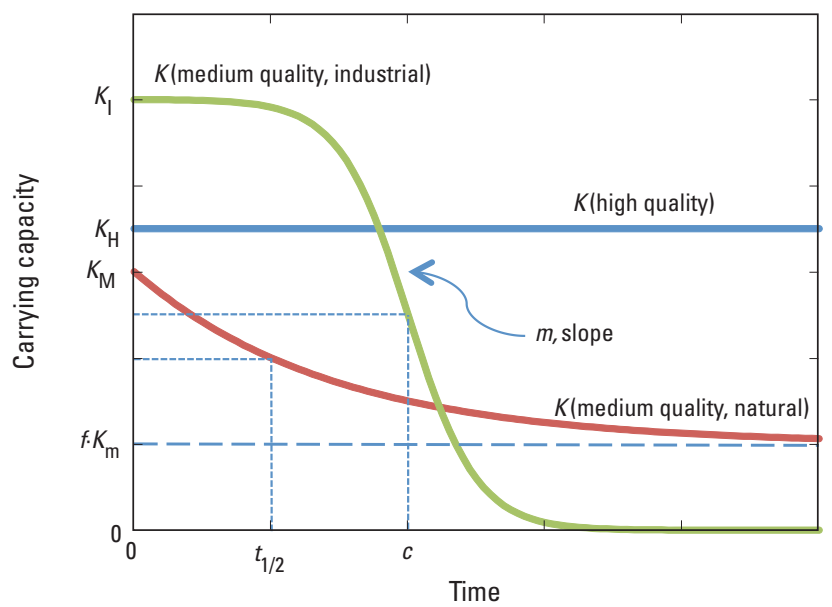

B. Upper St. Johns

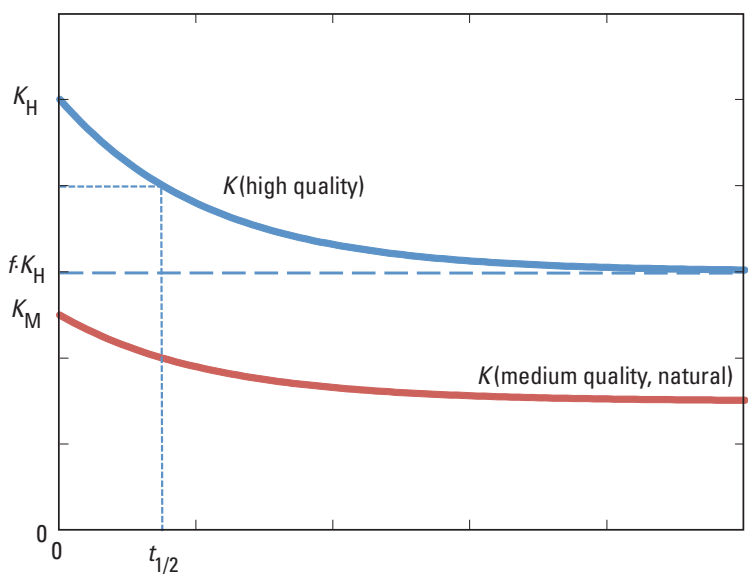

D. Atlantic

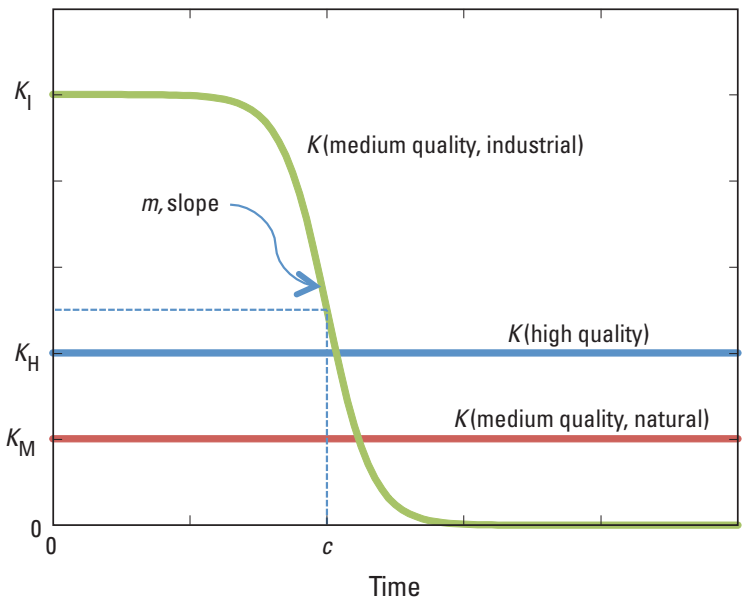

Figure 2. Models for Florida manatee carrying capacity as a function of time for four regions in Florida: $A$, Northwest; $B$, Upper St. Johns; $C$, Southwest; and $D$, Atlantic. The graphs show the functions used to project carrying capacity for high-quality warm-water habitat, medium-quality natural warm-water habitat, and medium-quality industrial warm-water habitat. The region-specific functions are described by the following parameters: $K_{\mathrm{H}^{\prime}}$ initial capacity in high-quality habitat; $K_{\mathrm{M}^{\prime}}$ initial capacity in medium-quality, natural habitat; $K_{\mathrm{l}}$, initial capacity in medium-quality industrial habitat; $f$, long-term fraction of carrying capacity provided by spring flow retained; $t_{1 / 2}$, half-life of carrying capacity loss associated with declines in spring flow; $c$, mid-point of industrial warm-water loss; $m$, slope of industrial warm-water loss. In the Northwest and Upper St. Johns regions, the long-term fraction of $K_{\mathrm{M}}$ retained is the same as for $K_{\mathrm{H}}$.

for their effect on adult survival; if the effects were significant, we developed baseline survival estimates from the models that included those effects (see next two paragraphs). For each region, the estimated annual survival rates (in the years without a red tide or cold effect) were used to calculate the baseline mean survival rate, and all estimated annual survival rates were used to calculate the temporal variance (Burnham and others, 1987). Although all available data were used to estimate survival, the last several annual survival rates are not estimable owing to bias at the end of the time series (Peñaloza and others, 2014; C.A. Langtimm, USGS, written commun., 2017).
Red-tide covariate. In order to test for effects of red tide on survival in the SW region, we developed a red-tide covariate for each year, based on the spatial extent, duration, intensity, and temporal relevance to manatees of red-tide blooms (Landsberg and others, 2009). After review of relevant red-tide data in the Florida Fish and Wildlife Research Institute (FWRI) Harmful Algal Bloom Monitoring Database, four criteria stood out as major factors in the expected lethal exposure of manatees in the SW: (1) whether red tide came inshore in three or more manatee foraging areas, (2) whether red tide remained inshore for greater than or equal to ( $\geq$ ) 100 days, (3) whether average daily maximum Karenia 
brevis (the dinoflagellate responsible for red tides) cell counts exceeded $10^{6}$ cells per liter, and (4) whether the red-tide event occurred in winter or spring when manatees regularly move between warm water and foraging areas. On the basis of these criteria, we included a red-tide covariate for five years (2002, 2003, 2005, 2006, and 2012) in the survival analysis for the SW. Two additional years $(1996,2013)$ also met these criteria but were not included in the survival analysis because of insufficient data for estimation.

Cold covariate. We developed a measure of the cumulative severity of a winter from the standpoint of a manatee, using a heating-degree-days metric relevant to manatee cold stress and mortality as a covariate for survival analysis (C.A. Langtimm, USGS, written commun., 2017; see also "Effects of Cold"). On the basis of this criterion, four years (1996, $2001,2003,2011)$ were identified as cold years in the AT region, and one (2010) was identified as a severely cold year in the AT region (see "Frequency and Magnitude of ColdRelated Mortality").

Calf survival rates. Manatee calves typically do not have enough scars or markings to allow them to be individually identified through MIPS, so their survival rates cannot be estimated using mark-recapture methods. In the earlier years of monitoring at the small aggregation in the USJ region, however, close observation from a canoe or kayak allowed observers to reliably identify calves by their shape, subtle markings, behavior, and associations with known adult females, providing data to reliably estimate calf survival (Langtimm and others, 2004). As in earlier versions of the CBM, first- and second-year calf survival rates were estimated for the NW, AT, and SW regions by comparison to the USJ region (Runge and others, 2004); beginning with CBMv5, however, the ratio of calf to adult mortality in USJ (rather than the ratio of calf to adult survival) was used to scale the corresponding ratio in the other regions.

Subadult survival rates. Similarly, few third- and fourth-year subadults have acquired unique identification scars for photo-identification and analysis. In the early USJ study, however, no difference in survival was found between subadults and adults (Langtimm and others, 2004). In the previous versions of the CBM, strict criteria for the adult class were maintained by excluding small, independent individuals that might have been subadults. We now think those animals are likely to be younger adults, and excluding them from the survival analysis could bias survival estimates toward older, experienced adults. Beginning with this revision of the CBM, we have pooled adults and the suspect subadult group.

\section{Reproductive Rates}

The breeding probabilities for females that have previously bred $\left(\gamma_{\mathrm{B}}\right)$ were estimated from MIPS data, which includes information on whether or not females are seen with a first-year calf, using multistate mark-recapture methods (Kendall and others, 2012). Note that in our analyses, the probability of breeding encompasses several aspects of reproduction: conception, gestation, parturition, and calf survival from birth to the calf's first winter (approximately 6 months). For two of the regions (NW, AT), the last few annual reproductive rates were not estimable owing to insufficient sampling effort. As with the survival rates, the mean and temporal variance of reproductive rates were estimated from the annual reproductive rates (Burnham and others, 1987).

In past analyses, information about mother-calf associations has not been available in MIPS for the SW region, so we either relied on reproductive histories of marked females in a small study in Sarasota Bay (Runge and others, 2004; Runge and others, 2007a) or simply assumed that the SW reproductive rates were the same as the AT rates (Runge and others, 2015). With this analysis, for the first time we have estimates of breeding probabilities $\left(\gamma_{\mathrm{B}}\right)$ for the $\mathrm{SW}$ region derived from the same data and analytical methods used for the other regions.

The data available to estimate the other two reproductive parameters $\left(\gamma_{4}\right.$ and $\left.\gamma_{\mathrm{p}}\right)$ is scant because it is rare to have the reproductive history for a known-age female beginning before her first parturition. Estimates for these parameters were obtained by calculating binomial proportions from the observed stage transitions of known, marked females (Runge and others, 2004).

The parameters that govern density dependence in the reproductive rates have not been measured in manatee populations. Estimates for $\alpha$ and $\beta$, the parameters of equation 1, were taken from Runge and others (2007a). The median value for $\alpha$ was 0.25 (range $0.15-0.50$ ) and the median value for $\beta$ was 2 (range 1-4). We assumed the initial population sizes were well enough below the carrying capacities that the reproductive parameters estimated from the mark-recapture analysis represented the reproductive rates at low density $\left(\gamma_{0}\right)$.

\section{Fractions of Mortality}

In order to model the effects of several of the threats in the CBM, an estimate of the fractions of mortality resulting from each of six causes (watercraft, water-control structures, marine debris, cold, red tide, and other) was needed. In addition, estimates of additional cold mortality (above baseline levels) by age class, winter severity, and warm-water habitat quality were needed. The carcass recovery and necropsy program run by FWC for the last 30 years provided the data for this estimation.

The analysis used 18 years of carcass recovery data from December 1, 1995, to November 30, 2013. For this analysis, a year extends from December 1 to November 30 (that is, the label 2001 applies to the period December 1, 2000-November 30, 2001). Carcasses less than $(<) 150$ centimeters $(\mathrm{cm})$ in length were assumed to be perinatal and excluded because their mortality is subsumed within the reproductive rate estimates (section "Reproductive Rates"), carcasses between 150 and $235 \mathrm{~cm}$ in length were considered calves, carcasses between 236 and $265 \mathrm{~cm}$ in length were assigned as subadults, and carcasses greater than $265 \mathrm{~cm}$ in length were assumed to 
be adults. The number of subadult carcasses was low, however, so subadults and adults were combined in the baseline fractions. For the variables where subadults and adults can differ, the superscript ${ }^{(j)}$ is used.

In addition to being divided by age class, year, and region, carcass recoveries were divided by warm-water habitat quality (high-, medium-, and low-quality). A 30-kilometer buffer (waterway distance) was used around the high- and medium-quality sites; carcasses recovered within those buffers were assigned to the corresponding habitat quality.

Analysis of fractions of mortality from carcass data is complicated by a number of hierarchical uncertainties (fig. 3)

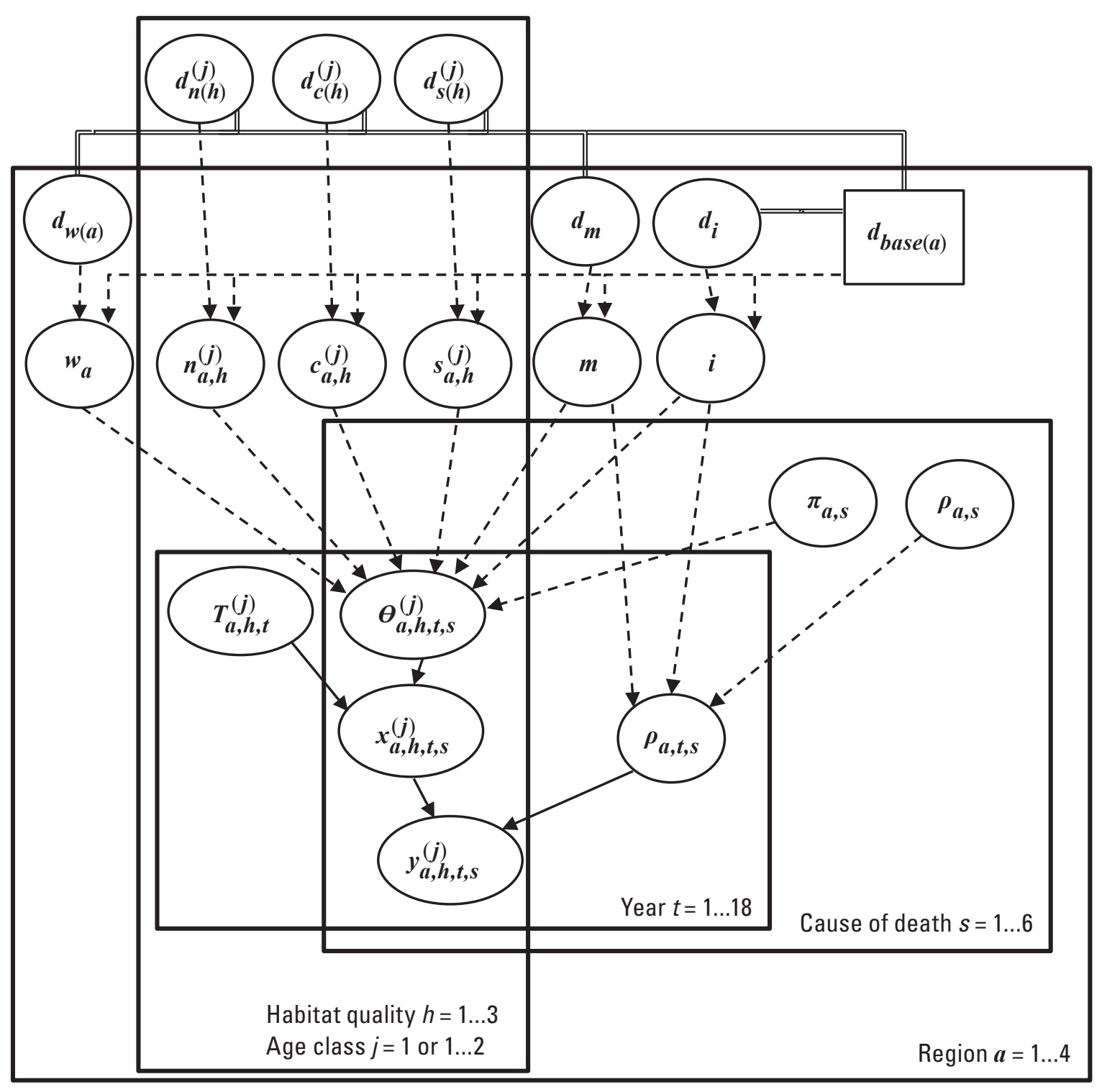

Figure 3. Directed acyclic graph of hierarchical model for estimating the fractions of mortality and additional mortality of Florida manatees associated with cold and red tide. Ellipses represent random variables (data or parameters), and small rectangles are constants. Large rectangles contain one or more index values. Solid arrows represent stochastic dependencies, dashed arrows represent deterministic dependencies, and double lines represent upper- or lower-bound dependencies (several variables have 1- $d_{\text {base (a) }}$, the baseline survival rate, as an upper bound). The variables are $d_{n(h)}^{(j)}, d_{c(h)}^{(j)}, d_{s(h)}^{(j)}, d_{w(a)}, d_{m}$, and $d_{i}$, the additional mortality rates for normal winters, cold winters, severe winters, water control structures, moderate red tide, and intense red tide, respectively; $n_{a, h}^{(j)}, c_{a, h}^{(j)}, s_{a, h}^{(j)}, w_{a}, m$, and $i$, the associated additional mortality factors, respectively; $\pi_{a, s}$ and $p_{a, s}$, the baseline fractions of mortality and probabilities of determination, respectively; $\theta_{a, h, t, s}^{(j)}$ and $\rho_{a, t, s}$, the year-, age-, and habitat-specific versions of the fractions of mortality and probabilities of determination; $T_{a, h, t}^{(j)}$, the grand total number of recovered carcasses; and $x_{a, h, t, s}^{(j)}$, and $y_{a, h, t, s}^{(j)}$, the total and determined number of carcasses from each cause, respectively. Calves and subadults/adults are modeled separately, so age class $j$ can have one possible value (calves) or two (subadults and adults). The red-tide rates and factors only apply to the Southwest region. 
and is most easily undertaken in a Bayesian framework. The primary uncertainty arises from the fraction of carcasses for which the cause of death cannot be determined. We accounted for this uncertainty by modeling the determined carcasses owing to cause $s$ in region $a$ in habitat quality $h$ for age class $j$ in year $t\left(y_{a, h, t, s}^{(j)}\right)$ as arising from a binomial distribution with two parameters: the number of recovered carcasses actually owing to cause $s$ ( $x_{a, h, t, s}^{(j)}$ an unknown quantity); and the probability of determination for that cause of death, year, and region $\left.\rho_{a, t, s}\right)$. The probability of determination is the proportion of carcasses for which the cause can be identified and may be different for different causes of death. The six cause-specific totals in a region, year, and warm-water habitat quality $\left(x_{a, h, t, s}^{(j)}\right)$ were modeled as a multinomial distribution with the grand total of carcasses from that region, year, and habitat quality (the sum of the determined and undetermined) and the fractions of mortality for that region, year, and habitat quality $\left(\theta_{a, h, t, s}^{(j)}\right)$.

The baseline fractions (our primary parameters of interest) and probabilities of determination by region and cause are defined as $\pi_{a, s}$ and $p_{a, s}$, respectively. The baseline fractions and probabilities of determination were given flat prior distributions.

We designated the years 2002, 2003, 2005, 2006, and 2012 as moderate red-tide mortality years in the SW region, the years 1996 and 2013 as intense red-tide mortality years in the SW, and the remaining years in the SW (and all years in the NW and AT regions) as baseline red-tide mortality years (see "Survival Rates" in the "Methods" section). In a moderate red-tide year, an additional mortality factor $(m)$ was added to the red-tide fraction, then all fractions were divided by that factor plus 1 (to keep the fractions summing to 1 ). This formula follows from the assumptions that baseline mortality rates are constant in years without additional mortality factors and that mortality causes are additive (in other words, if the cold mortality rate goes up for a particular year or habitat type, the entanglement mortality rate will remain unchanged). The moderate red-tide additional mortality factor was defined as the moderate red-tide additional rate $\left(d_{m}\right)$ divided by the baseline SW mortality rate $\left(d_{\text {base (SW) }}\right)$. The baseline mortality rate is the additive inverse of the estimated survival rate (see "Survival Rates" in the "Methods" section). The prior distribution for the additional mortality rate was a uniform distribution between 0 and the survival rate (to ensure that the total mortality rate does not exceed 1). The same process was used to model the relation between the additional mortality factor (i) and additional red-tide mortality rate $\left(d_{i}\right)$ in intense red-tide mortality years. Estimates of annual survival vary between first- and second-year calves. To estimate a baseline mortality rate for calves overall, the geometric mean of survival rates was used.

Similar to the red tide additional mortality factors ( $m$ and $i$ ), there are factors for the effects of cold and severely cold winters on cold mortality $\left(c_{a, h}^{(j)}\right.$ and $s_{a, h}^{(j)}$, respectively) that are specific to the warm-water habitat type $h$ where the carcass was recovered, the year $a$, and the age class $j$. We used the same covariate as the survival analysis to designate cold and severely cold years (see "Survival Rates" in the "Methods" section). For adults, the additional cold mortality factor is set to 0 for high- and medium-quality habitats because this is already factored into the survival rate analysis; for calves and subadults, it is set to 0 for high-quality habitats for the same reason. In addition, for calves and subadults there is a normal winter additional cold mortality factor $\left(n_{a, h}^{(j)}\right)$ that applies to low-quality habitat for subadults and to low- and mediumquality habitat for calves. Therefore, the baseline fractions of mortality $\left(\pi_{a, s}\right)$ are not changed by cold factors in the following cases: for adults, in normal winters in all habitats and cold winters in high- and medium-quality habitats; for subadults, in normal winters in high- and medium-quality habitats and cold winters in high-quality habitats; and for calves, in normal and cold winters in high-quality habitats. The additional cold factors affected the specific fractions of mortality $\left(\theta_{a, h, t, s}^{(j)}\right)$ in a manner similar to that of the additional red-tide factors. We also estimated additional mortality rates resulting from normal, cold, and severely cold winters $\left(d_{n, h}^{(j)}, d_{c, h}^{(j)}\right.$, and $\mathrm{d}_{s, h}^{(j)}$, respectively) and derived the additional mortality factors from them in a manner similar to that for the red-tide additional mortality rates and factors.

In 1994, FWC, in conjunction with the Florida Department of Environmental Protection, began implementing measures to reduce the number of deaths caused by water-control structures by retrofitting gates and locks to remain open when blocked by large objects, including manatees. By the end of 2004, 23 structures (out of 33 targeted) had been retrofitted or closed; the first 9 retrofittings, which included the most lethal structures, were completed by the end of 2000 (Florida Fish and Wildlife Conservation Commission, 2007). Thus, we viewed year 2000 as a median point in the recent reduction in water-control structure mortality. In order to account for this mitigation in the analysis, subadult and adult carcasses pre2001 were modeled with an additional mortality factor $\left(w_{a}\right)$ similar to the others. Because few calf deaths and few deaths in the NW region were attributed to water-control structures over the entire period, this mitigation effect was not modeled for calves or for the NW region. The analysis was conducted using the NIMBLE package (a version of the BUGS language) in program R (Lunn and others, 2000; R Development Core Team, 2015; NIMBLE Development Team, 2016).

\section{Effects of Red Tide}

The spatial extent, temporal persistence, and magnitude of red tide, along with its effects on manatees, are monitored by FWRI. From these data, the historical patterns of the effects of red tide on manatees can be estimated, but it is not known whether the historical patterns will hold in the future. Thus, a formal expert judgment process was conducted to estimate probability distributions for the frequency and relative magnitude of red-tide-related manatee mortality events over a 100-year time horizon in each region (Julien Martin, USGS, written commun., 2017). A panel of 12 experts in manatee biology and red tide ecology was convened, and the 
Investigate-Discuss-Estimate-Aggregate protocol was followed to structure the expert judgment (Hanea and others, 2017). During two rounds of elicitations, each expert provided a best estimate value and plausible low and high values (that is, bounding a confidence level of $80 \%$ ) for each parameter in each of three regions of the Florida manatee's range (NW, AT, and SW) for two periods ( $0-40$ and $41-100$ years from the start of the modeled simulations, 2011). Probability distributions were fitted for each parameter, time period, and expert using the three elicited values. The parameter estimates elicited from individual experts were aggregated, and a parametric distribution was fitted to the aggregated results. Data were not available to estimate the correlation in red-tide years among regions (the parameters of the Gaussian copula); these correlations were all assumed to be 0.5 (Julien Martin, USGS, written commun., 2017).

Estimates of additional red-tide mortality in moderate years in the SW for subadults and adults were obtained from the survival rate analysis (see "Survival Rates" in the "Methods" section). Estimates of additional red-tide mortality in the SW for calves in moderate and intense years and subadults and adults in intense years were obtained from the fractions of mortality analysis (see "Fractions of Mortality" in the "Methods" section).

\section{Effects of Cold}

In CBMv6, the effects of cold-related mortality are governed by two sets of parameters: a set of frequencies (for the occurrence of cold or severe cold) and a set of 27 additional mortality rates (for each of 3 age classes in each of 3 types of habitat in each of 3 types of year). The estimation of the additional mortality rates was integrated with the estimation of the fractions of mortality and is explained in the "Methods" section "Fractions of Mortality."

To estimate the future frequency of cold-related events, we wanted first to define a cold or severely cold year on the basis of climate variables rather than on observed cold-related deaths. We developed a measure of the severity of a winter, using a heating degree days metric relevant to manatee cold stress and mortality. The metric, tdef 14 , calculates a daily mean air temperature by averaging the daily high and low temperatures at a particular weather station, subtracts 14 degrees Celsius $\left({ }^{\circ} \mathrm{C}\right)$ from the daily mean, and sums all the negative daily differences from November 1 through March 31 . Thus, the tdef 14 metric is the number of degree days less than $14{ }^{\circ} \mathrm{C}$ from November 1 to March 31 and has units C-day. Using historical weather records (1950-2013) at selected National Weather Service stations in each of the four regions, we calculated the annual tdef14 and classified years as severely cold if the tdef 14 was less than the 5-percent quantile for that region, cold if the quantile was between 5 and 20 percent, and normal if the quantile was above 20 percent. That is, the thresholds were defined so that the frequency of severe cold and cold were 5-percent and 15-percent, respectively, in all regions.
The correlations in cold years among regions (the parameters of the Gaussian copula) were estimated by finding the correlations in tdef14 among regions (1950-2013).

Two hypotheses about the future frequency of cold events were considered: that the future frequencies would match the historical frequencies and that the future frequencies would be elevated. To estimate the historical frequencies, the weather data during 1950-2013 were used. To estimate the elevated frequencies, the frequency of cold and severely cold years across all four regions during 2001-11 was considered.

\section{Effects of Emergent Disease}

We considered the emergence of a virulent, infectious disease as a rare but possible catastrophe with potentially large effects on survival and reproductive rates. No epizootics have been documented in manatees, but manatees have been exposed to pathogens, such as morbillivirus (Duignan and others, 1995), that have caused major die-off events in other marine mammals (Lipscomb and others, 1994). The tendency of manatees to form large aggregations at warm-water sites during winter cold periods also makes them vulnerable to the spread of an infectious disease. A review by Harwood and Hall (1990) on the population effects of such epizootics in marine mammals provided a useful guide for forming plausible estimates for frequency and magnitude of effects on survival and reproduction. Historical data on seal epizootics in the United Kingdom, for example, show that such events occurred about every 50 years, for a frequency of 0.02 (Harwood and Hall, 1990). Those authors concluded, "it is clear that marine mammal populations are occasionally subject to events that may remove $50 \%$ or more of the individuals" (Harwood and Hall, 1990, p.255). They suggest that average mortality may be about 15 to 30 percent higher in epidemic years. We chose not to model the worst-case scenario documented in other marine mammal species because a severe disease outbreak has not yet been documented for sirenians. We set the frequency of this type of catastrophe at a median of 0.01 , ranging from 0 to 0.02 , using a two-phase uniform distribution (Runge and others, 2007a). Survival and reproductive rates were reduced independently by a median of 25 percent using a two-phase uniform distribution with a range of 0 to 50 percent. No correlation between regions was included.

\section{Carrying Capacity}

Over a period of 6 months during 2011-12, an expert panel was convened to estimate the current and future carrying capacity in each of the four regions (M.C. Runge, USGS and C.J. Deutsch, FWRI, written commun., 2017).Twentythree experts from 9 agencies (USGS, FWS, FWC, Progress Energy, Florida Power and Light, Sea to Shore Alliance, National Aeronautics and Space Administration, Mote Marine Laboratory, St. Johns River Water Management District) were invited to participate in the process, based on their expertise 
in manatee use of warm-water habitats or their expertise in the status of, and factors affecting, warm-water habitat. The process was designed to preserve the independence of experts and harness the insights from a group of experts while guarding against cognitive biases and group dynamics that undermine expert judgments (Burgman and others, 2011; Hanea and others, 2017). During the pre-elicitation phase, experts were selected, the problem was defined and framed, and training in cognitive bias and expert elicitation was provided. Importantly, during the pre-elicitation phase, the structure of the carrying capacity models (fig. 2) was fully developed and discussed by the experts. During the elicitation phase, individual experts initially provided estimates for the parameters independently using a four-point method (Speirs-Bridge and others, 2010). These results were compiled and presented graphically to the experts with the anonymity of the individual results retained. We facilitated discussion with the group of experts about their initial results to clarify concepts, to present summary data (for example, from water-temperature monitoring and aerial surveys), and to share insights and approaches to the problem. The experts then individually provided a second set of estimates using the four-point method. Four months later, with the results of a site-specific assessment of carrying capacity to provide new insight (Provancha and others, 2012), we discussed the second round of elicitation and asked the experts to provide a third and final set of estimates. During the post-elicitation phase of the process, the experts' judgments were aggregated. First, for each parameter and for each expert separately, we found the probability distribution that best fit the quantiles provided in the four-point elicitation, as measured by the Kullback-Leibler (KL) divergence (Kullback and Leibler, 1951). Second, for each parameter, we averaged the probability density functions across the experts, with equal weighting. Third, we found the probability distribution that best fit the average aggregated distribution by minimizing the KL divergence.

\section{Initial Abundance}

Estimates of initial abundance and associated uncertainties were based on the abundance survey conducted during 2011-12 (Martin and others, 2015). Unlike synoptic surveys, the estimator considered two important sources of error: spatial variation in abundance and imperfect detection. Lognormal distributions were used to express uncertainty in the abundance estimates for AT, SW, and NW. For the NW region, the posterior mean abundance, rounded to the nearest 10 , was 610 (standard deviation [SD] 150, mean on log scale 6.383, $\mathrm{SD}$ on log scale 0.241 ). For the SW region, the posterior mean was 2,180 (SD 310, mean on log scale 7.675, SD on log scale 0.144$)$. For the East coast of Florida, the posterior mean population size was 3,560 (SD 400); this included animals in both the AT and USJ regions. Because a substantial number of manatees either left the USJ region at the time of the survey or were undetected during the abundance survey (for example, because of vegetation cover that makes counting particularly difficult in this region), ground counts conducted at Blue Spring during the winter were used to estimate abundance in the USJ. Uncertainty was expressed with a uniform distribution between 293 (the maximum daily winter count; Wayne Hartley, Save the Manatee Club, unpub. data, 2012) and 397 (the total number of individuals sighted during October 2011April 2012; Wayne Hartley, unpub. data, 2012). The estimate for the AT (posterior mean 3,220, SD 400, mean on log scale 8.070 , SD on log scale 0.123 ) was derived by subtracting the USJ estimate from the East coast estimate using a Monte Carlo simulation approach.

\section{Scenario Design}

\section{Baseline and Scenario Philosophy}

All of the earlier runs of the CBM used a baseline-andscenarios approach, in which one scenario (the baseline) represents the best single summary of the available information about the status of the subspecies and the threats it faces over the next century or so. Additional scenarios explore a number of questions to better elucidate the nature and strength of the threats. In 2007, scenarios were used to produce the "threats analysis," which allowed a quantification of the relative strength of the various threats (Runge and others, 2007b). Scenarios also can be used to conduct sensitivity analysis, to simulate different hypotheses about underlying dynamics, or to explore the ramifications of possible emerging threats.

\section{Baseline Scenario}

As part of this process, the definition of the baseline scenario is important and requires a number of careful judgments of scientific and policy natures. The approach we have always taken is that the baseline scenario represents the incorporation of the best available scientific and commercial information and the most appropriate interpretation of future threats for assessment of status under the ESA. Several considerations arise from this policy context for developing the baseline scenario. First, because we interpreted status assessment under the ESA as a risk analysis, very careful attention was paid to incorporation of uncertainty into the parameter estimates and structure of the model. To the extent possible, we want the results to integrate all that uncertainty, so the management agencies that use the results can take into account the risk posed by that uncertainty. Where it was not practical to express uncertainty in a parameter or structure within the model, an effort was made to use point estimates that reflect the risk standard of the ESA, which was interpreted to mean erring on the side of conservation of the species. Second, with regard to representing future threats, Factor D ("the inadequacy of existing regulatory mechanisms," 16 U.S.C. $§ 1533(\mathrm{a})(\mathrm{D}))$ plays a role. For example, as of 2016, there are many power plants with National Pollutant Discharge Elimination System permits that include an allowance for once-through cooling, which provides warm-water habitat for manatees in the winter. We 
assume, however, that warm-water habitat will only be available until the end of the operational lifetime of each existing plant. Although it is possible that the plants will be repowered and permitted to continue to provide warm-water effluent, the existing regulatory mechanisms do not assure us of that.

The baseline scenario relies on empirical evidence from historical data but is not bound to it. The CBM produces a forecast, meaning that how it represents the future, not the past, is its most important feature. In many cases, historical data provide us with an excellent understanding of manatee population dynamics, but there are circumstances in which the best available evidence leads us to believe that the future will not be like the past. Thus, the baseline scenario needs to contain the best scientific assessment of what the future will be like, given the available evidence. This almost always requires a mixture of empirical analysis and expert judgment. For example, in CBMv5 (Runge and others, 2015), we estimated the magnitude of red-tide mortality in the SW from a mark-recapture analysis of historical survival rates, but an expert panel estimated the frequency of future red-tide events (the panel estimated an increase in those rates). As another example, in estimating the fractions of mortality associated with various causes of death, pre-2001 mortalities were separated from post-2001 mortalities. Only the latter was used for the baseline because most gates and locks were retrofitted to reduce manatee mortality during the early 2000s; we believe the data prior to 2001 no longer provide a reasonable estimate of the future threat from water-control structures.

The current version of the CBM (CBMv6) uses these same considerations to establish the baseline scenario. Several recent events, however, have raised questions about how to establish the baseline scenario, namely, the cold-induced mortality events of 2009-10 and 2010-11, the red-tide mortality event of 2013, and others. At this time, it is difficult to know whether those represent odd one-time events, represent rare events likely to remain rare in the future, or represent emerging threats. In consultation with other scientific experts and with management agencies, we have attempted to navigate this uncertainty and have made our collective best judgments about what to include in the baseline scenario. In the section "Potential Emerging Threats," however, we describe how the possible ramification of alternative assumptions was explored.

The features of the baseline scenario include seven assumptions. (1) Watercraft-related mortality will maintain its historical rate over the indefinite future, based on the assumption that current protections (for example, speed zones) or their equivalent will be maintained even if there is a change in conservation status or a change in authority to implement protection. If the population size continues to increase, watercraftrelated deaths will increase in proportion, but the proportion will stay constant. (2) Warm water produced at power-generation facilities, and the carrying capacity it represents, will not be replaced at the end of the current expected operating life for each power plant. Although it is conceivable that power companies will seek to continue the use of once-through cooling if and when they refurbish or rebuild old facilities, there is no regulatory guarantee such an exception will be granted, so it was assumed that the manatee carrying capacity associated with these particular warm-water sources will be lost. (3) The carrying capacity provided by natural spring flows will be reduced over the long term at the rates estimated by the expert panel. Behind this forecast is an assumption that the human population in Florida will continue to grow and will continue to increase its water use. (4) As the manatee population increases and as the carrying capacity decreases, manatees will distribute among high-, medium-, and low-quality sites using one of three rules (thermal selection rule, ecological trap, historical) with most weight on the historical rule. (5) Cold and severely cold years will occur with the same frequency as in the last 6 decades (1950-2013). (6) Red-tide mortality events will occur at a considerably higher frequency than in the past; the appearance of intense red-tide events is possible. (7) The phenomenon in the IRL is a short-lived event that will not persist as a chronic source of mortality.

\section{Threats Analysis}

A large number of scenarios was run to examine the effects of the various threats on long-term persistence of Florida manatees. These scenarios either removed or accelerated existing threats.

For three of the threats (mortality owing to watercraft, water-control structures, and entanglement by marine debris), the threat was "removed" by reducing the regional mortality of adults, subadults, and calves by the fractions estimated from the Bayesian fractions-of-mortality analysis. This assumes that the causes of mortality are additive, not compensatory. For instance, in a particular replicate, if the baseline scenario had an adult survival rate of 0.94 and the fraction of adult mortality owing to watercraft was 40 percent, then the "no watercraft mortality" scenario used an adult survival rate of 0.964 (this is a $40 \%$ interpolation between 0.94 and 1.0). To account for uncertainty, each replicate has a different base survival rate and fraction of mortality, based on the sampling distributions for each. The fractions of adult mortality were applied to the survival rates for subadults (age $3+$ ) and adults; the fractions of calf mortality were applied to the survival rates for firstand second-year calves. Note that the fractions of mortality owing to background cold and background red tide were treated as part of the baseline mortality and were not removed in any of the scenarios.

For the threat owing to watercraft, partial reductions and increases of watercraft-related mortality were considered. The partial reduction was implemented with a proportional reduction in the mortality, whereas an increase was modeled by assuming that a proportional increase in mortality rate would occur gradually over a 30-year period, then stabilize.

For the threat resulting from loss of warm-water habitat, the threat was removed from the model by maintaining winter warm-water capacity at current levels for the indefinite future rather than having that capacity drop at the currently anticipated rates. For manatees that rely primarily on 
first-order springs (USJ and NW), this could happen either by preservation of existing spring flow and protection (through management of minimum flow levels, such as those proposed for Blue Spring [Rouhani and others, 2007]) or by mitigation that exactly matches the anticipated loss (for example, through increasing access to and protection of other springs). For manatees that rely primarily on industrial warm-water effluents (AT and SW), this could happen by maintaining those industrial effluents at their existing levels, through restoration of natural habitats in those areas, or by replacing lost warmwater capacity using alternative approaches (for example, creating basins warmed by ground water). Note that in the scenario that removed this threat, cold-related mortality owing to loss of warm-water capacity was reduced, but background, density-independent cold-related mortality (as captured in the fractions-of-mortality analysis) remained. To model a partial reduction of this threat, the long-term loss in warm-water capacity was reduced proportionally, but the other parameters describing the loss (for example, its timing) were maintained. Two scenarios of accelerated loss of industrial warm-water capacity were considered: the immediate loss of warm-water effluent produced by coal-fired power plants and the immediate loss of all industrial warm-water carrying capacity. Coal-fired power plants currently represent 100 percent of the industrial carrying capacity in the NW and approximately 50 percent of the industrial carrying capacity in the SW region. In the NW, the Duke Energy Crystal River Complex has four coal-fired units, and because the nuclear unit is being decommissioned, the coal units represent all of the industrial warm water in this region. In the SW, Tampa Electric Company's Big Bend plant has four coal-fired units that represent about one-half of the industrial warm-water capacity in the region.

For red tide, the threat was removed from the model by setting the probability of occurrence of moderate and intense red-tide events to zero. Background levels of red-tide mortality occur every year and are incorporated into the baseline estimates of survival.

\section{Potential Emerging Threats}

As discussed in the section "Current Questions," the series of mortality events between 2009 and 2013 raised questions about whether there are new threats emerging as a result of changes in the environment in coastal Florida. At this time, we do not have the scientific evidence to fully understand the causes and mechanisms of these events, so we investigated their possible effects by considering a series of hypotheses, each of which was implemented as a separate scenario in CBMv6.

Hypotheses regarding cold-related mortality. As noted in "Current Questions," four hypotheses were considered regarding the mechanism that gave rise to the cold-related mortality events of 2009-10 and 2010-11. To implement hypothesis 1, which posits that the cold-related mortality events were simply back-to-back cold years, we used the historical frequencies for cold and severely cold years, along with the expert-elicited estimates of carrying capacity. To implement hypothesis 2 , which posits that the cold-related mortality events arose because the warm-water carrying capacity had been exceeded, when sampling from the distributions that represented uncertainty in the initial carrying capacity, only those values that were less than or equal to the initial population size were allowed, thus forcing all replicates to begin at or greater than carrying capacity. The future frequency of cold was set at the historical frequencies. To implement hypothesis 3 , which posits that the cold-related mortality events were unusual densityindependent events, the historical frequencies of cold and the expert-elicited estimates of carrying capacity were used, but the first 2 years of the simulations were forced to be severely cold, and all the animals were placed in low-quality warmwater habitat. To implement hypothesis 4 , which posits that the cold-related mortality events of 2009-11 are harbingers of more frequent cold events in the future, we assumed the frequency of cold and severely cold years in the future would be at the elevated rate seen during 2001-11 rather than the historical rate seen during 1950-2013. Hypothesis 1 was used in the baseline scenario for three reasons: the winters of 2009-10 and 2010-11 were not unexpected, based on the historical climate record (a log-normal distribution fits the 1950-2013 time series of tdef14 without outliers); long-term climate forecasts do not predict increased frequency of cold-air outbreaks in the southeastern United States (Gao and others, 2015); and the expert panel consulted on manatee carrying capacity did not think it was likely that manatees are currently limited by warm-water habitat.

Hypotheses regarding red-tide mortality. As noted in "Current Questions," four hypotheses were considered regarding the character and frequency of red-tide mortality events in the future. For hypotheses 1 and 3 (which posit that no new class of red-tide year is emerging), two classes of red-tide years (background years, red-tide years) were used, whereas for hypotheses 2 and 4 (which posit that a third intense class of red-tide year can occur), three classes of red-tide years (background, moderate, and intense) were used. For hypotheses 1 and 2 (which posit that future red tide will occur at the same frequency as in the past), historical frequencies of redtide mortality events were used; for hypotheses 3 and 4 (which posit an increase in the future frequency of red tide), expertelicited frequencies of red-tide mortality events (the medians of which were elevated above historical levels) were used. All the experts on the red-tide panel favored the hypothesis that red-tide mortality events would probably increase in the future, so for the baseline scenario a combination of hypotheses 3 and 4 was used, weighted by the proportions of experts that favored the 2- and 3-class hypotheses.

Chronic Atlantic mortality. This scenario is motivated by the IRL UME (see "Current Questions") but includes the assumption that such a loss of individuals would continue to occur indefinitely in the AT region with the mortality occurring without regard to density or stage class and at a regional rate $(2 \%)$ somewhat higher than indicated by the ratio of the number of carcasses recovered during the IRL UME to the 
size of the AT population. For this to occur, there would need to be a behavioral mechanism that continued to draw manatees into a mortality sink caused by an environmental factor they could not detect or did not perceive as a threat. We think this is a worst-case scenario based on the IRL UME.

Multiple emerging threats. As an endpoint to bracket the range of scenarios considered in this analysis, this scenario assumes the simultaneous emergence or strengthening of a number of threats. This scenario does not represent the bestjudgment baseline, but rather a possible, if unlikely, concurrence of many adverse conditions. The scenario includes seven features. (1) The watercraft-related mortality rate increases by 50 percent over the next 30 years, then stabilizes. (For example, in adults in the AT region, the mean watercraft-related mortality rate would increase from 0.021 to 0.032 over the first 30 years of simulation). This is motivated by an expected human population growth in Florida of around 40 percent between 2010 and 2040 (Rayer and Wang, 2016) with a higher proportion living near the coast and having boats, with fuel prices staying low, and with tourism in the State continuing to increase. (2) Immediate loss of coal-fired power plants, representing 50 percent of the industrial warm-water carrying capacity in the SW and 100 percent of the industrial warmwater carrying capacity in the NW. This part of the scenario assumes that the increase in the availability of renewable power will continue to put economic pressure on coal plants, and the companies will choose not to convert these units to other sources of fuel. (3) Reduction of carrying capacity provided by natural spring flows of 50 percent over the long term. This level of reduction in spring flow is the greatest reduction we analyzed and would represent the pessimistic end of what the expert panel considered. If water-withdrawal rates increase with increased demand from a larger human population and rainfall decreases, this scenario is deemed possible. (4) Historical warm-water distribution rule. This scenario incorporates the assumption that manatees choose warm-water sites in proportion to their historical use, which was the consensus of the experts consulted. (5) The frequency of cold and severely cold years (cold hypothesis 4) is elevated. (6) The frequency of moderate and intense red tide is elevated (red-tide hypothesis 4). (7) Chronic density-independent additional mortality occurs in the AT region (2\%).

\section{Sensitivity Analysis}

In CBMv6, uncertainty in all parameters and uncertainty about some of the structural elements were accounted for by sampling from those uncertainties at the beginning of each replicate. In developing the uncertainty distributions, we made an effort to fully capture the range of possible dynamics, based on the best available information. But with regard to two aspects of the carrying capacity dynamics, the warmwater expert panel indicated that the available information was scant. For these aspects of the model, we wanted to specifically see how that uncertainty affected the results. These two sensitivity analyses do not represent forecasts or hypotheses about potential emerging threats; rather, they are simply an effort to see whether the results are robust to uncertainty in these dynamics.

Sensitivity to long-term loss of carrying capacity at springs. The future loss of warm-water habitat at springs is difficult to forecast because it is related to the complex interactions among climate change, urbanization, water-use regulation, and enforcement. The baseline scenario uses the expertelicited estimates for this loss, but these estimates encompass a wide range of uncertainty. The expert panel expected carrying capacity at springs to decrease at high- and medium-quality natural sites in the NW and USJ regions and at mediumquality natural sites in the SW region; the expert panel did not expect such loss at high-quality sites in the SW region or at any natural sites in the AT region. For the sensitivity analysis, for those types of natural sites at which the experts expected change, the long-term change in carrying capacity was set at the following fixed values: -50 percent, -20 percent, -10 percent, and +10 percent.

Sensitivity to the behavioral rule used by manatees to choose warm-water sites. The baseline scenario used a weighted mixture of the thermal quality selection, ecological trap, and historical distribution rules to allocate animals to warm-water habitat types, weighted heavily toward the latter rule. For the sensitivity analysis, each behavioral rule was run separately in CBMv6.

\section{Output Metrics}

For each scenario, we ran 5,000 replicates over 150 years; thus, for each point in time, a distribution of population size (and structure) was produced that integrates the uncertainty over parameters and stochastic events. From the results, we graphed the distribution of the population size against time, for the total population and for adults only, at three spatial scales (region, coast, and statewide). In the population model, adults include females 4.5 years and older that have previously bred and males 4.5 years and older.

As a summary metric relevant for assessment under the ESA, the probability of quasi-extinction over the ensuing $y$ years was calculated, where quasi-extinction is defined as an adult population size of fewer than $z$ on either the East coast or the Gulf coast. The East coast consists of the USJ and AT regions, and the Gulf coast consists of the NW and SW regions. The probability of quasi-extinction was calculated for three periods $(50,100$, and 150 years) and three quasi-extinction thresholds (100, 250, and 500 adults).

The expected minimum population size (EMP) is reported as a measure of the propensity for decline (McCarthy and Thompson, 2001). In cases where the risk of extinction is small, the probability of extinction or quasi-extinction is determined by a small number of the replicates in a simulation, so it can be difficult to estimate. The EMP uses information from all of the replicates and so provides a stable metric of risk. The 
EMP was calculated by finding the minimum total population size on either coast over a specified time period for each replicate, then taking the average of the minimum values.

Summary metrics that are relevant for classification under the IUCN Red List (IUCN, 2012) also were calculated. All of these metrics are the probability of the adult population declining by at least 10,30 , or 50 percent over either 60 years (3 generations) or 100 years. The metrics were calculated for each region, each coast, either coast, and statewide. To generate these metrics, the population size at the end point of each replicate was compared to the initial population size for that replicate and the proportional magnitude of decline was calculated; then, the proportion of replicates that exceeded the threshold magnitude was computed.

\section{Results}

The subsections that follow describe the results from new and updated parameter estimation, baseline projections from CBMv6, an analysis of threats, examination of potential emerging threats, and several sensitivity analyses for the Florida manatee.

\section{Parameter Estimation}

This comprehensive revision of the CBM generated new estimates for many of the parameters, as well as new output from the model itself. The new parameter estimates are summarized here.

\section{Survival Rates}

The baseline adult survival rates for each region are shown in table 1 . The mean values of survival and their precision are comparable to estimates for CBMv5 in 2012 (Runge and others, 2015), but the means are higher and considerably more precise than the estimates for CBMv4 in 2007 (Runge and others, 2007a). The negative bias present in earlier estimates has been reduced with a larger sample size, a longer time series, and the use of the Barker/RD model to account for processes that can bias estimates (Langtimm and others, 2016). The use of auxiliary sightings has increased the precision (Kendall and others, 2013). The variation among regions in baseline years is small

The effects of two covariates on adult survival rates were examined. A significant additive effect in the $\mathrm{SW}$ from moderate red tides $(-0.0184$, standard error [SE] 0.0050) reduced mean survival from 0.976 (SE 0.002) in background red-tide years to 0.958 (SE 0.005) in the five moderate red-tide years (2002, 2003, 2005, 2006, and 2012). Effects on survival owing to moderate or severe cold were not evident in the SW or USJ regions. In the NW region, moderate and severe cold also showed no effect on survival, although an annual estimate of survival was not available for one of the recent severely cold years (2010). In the AT region, there was no effect on survival owing to moderate cold. There was an apparent additive effect on survival for severe cold. However, this effect was based on a single severely cold year (2010) at the end of the estimable time series, and subsequent analysis indicated it was biased. Additional monitoring data could resolve the question of effects from severe cold in 2010 for all regions.

Table 1. Baseline Florida manatee mean survival and reproductive rates for four regions in Florida, 1982-2015.

[The survival rates are for baseline conditions and do not include the effects of cold or red tide. In the column labeled "Data included," the years for which photo-identification data were included are shown; 1987, for instance, means sampling was conducted during winter 1986-87. Annual survival and reproductive rates are not always estimable, owing to bias at the end of the time series or other sampling issues. SE, standard error of the mean]

\begin{tabular}{lcccc}
\hline \multicolumn{1}{c}{ Region } & Mean rate & SE & Data included & Years estimable \\
\hline Atlantic & \multicolumn{3}{c}{ Adult survival rates } & \\
Upper St. Johns & 0.9729 & 0.0029 & $1987-2014$ & $1987-2010$ \\
Northwest & 0.9790 & 0.0031 & $1987-2014$ & $1987-2011$ \\
Southwest & 0.9780 & 0.0026 & $1982-2014$ & $1982-2009$ \\
& 0.9759 & 0.0023 & $1996-2015$ & $1997-2012$ \\
\hline Atlantic & & Reproductive rates & & $1987-2010$ \\
Upper St. Johns & 0.307 & 0.023 & $1987-2014$ & $1997-2013$ \\
Northwest & 0.384 & 0.022 & $1997-2014$ & $1987-2010$ \\
Southwest & 0.368 & 0.027 & $1987-2014$ & $1998-2014$ \\
\hline
\end{tabular}




\section{Reproductive Rates}

Regional mean reproductive rates are shown in table 1 . The mean probability that a female who did not breed the previous year will breed the following year is more than 30 percent for all regions. Breeding rates were higher in the USJ and NW regions than in the more developed SW and AT regions. Compared to the adult survival rates, the reproductive rates have a higher standard error (table 1) and greater temporal variance (C.A. Langtimm, USGS, written commun., 2017). This pattern of low variability in adult survival and high variability in reproduction is the typical life-history strategy for large, long-lived mammals.

\section{Fractions of Mortality}

The analysis included 5,544 recovered carcasses, of which 1,949 were calves, 1,002 were subadults, and 2,593 were adults. By region, 234 carcasses were from NW, 126 from USJ, 2,713 from SW, and 2,471 from AT. The cause of death was not determined for 1,790 or 32 percent of the carcasses.

Watercraft-related mortality was always estimated as the leading cause of death, except for calves in the SW region where cold-related mortality was slightly higher (fig. 4). The estimated fractions of mortality owing to watercraft ranged across regions from 0.29 to 0.60 for calves and 0.40 to 0.79 for adults and subadults. The estimated fractions owing to the other anthropogenic causes (water-control structures and marine debris) were much smaller (always under 0.07). The fractions of mortality owing to red tide were minimal except in the SW region where the estimated background fractions of mortality from red tide were 0.142 and 0.107 for subadults/ adults and calves, respectively (fig. 4). The uncertainties in the estimates were generally larger for calves than for the older age class and larger for the USJ and NW regions than for the AT and SW regions, all owing to differences in sample size.

The estimated additional mortality rates from red tide events were higher for calves than for subadults/adults and more than four times higher in intense red-tide years than in moderate red-tide years (fig. 5). The estimate of subadult/ adult additional mortality in moderate red-tide years $(0.015)$ was similar to that from the survival analysis for those years (0.018, see "Survival Rates" in the "Results" section).

\section{Frequency and Magnitude of Red-Tide Mortality}

The panel of experts convened to estimate the frequency and relative magnitude of red-tide mortality self-identified into two groups (Julien Martin, USGS, written commun., 2017). One group believed there are only two classes of years (background and red-tide years), and the other group thought the data were best explained by three classes of years (background, moderate red-tide, and intense red-tide years). The median estimates of the frequency of red-tide mortality events were greater than the historical frequencies, and the credible intervals were wider (table 2). The model allows the frequencies of red tide to be different in years $0-40$ than in years $41-150$ of the simulations; table 2 shows the frequency for years 0-40 from 2011. In most cases, the expert-elicited frequencies for the years 41-100 years from 2011 (not shown) did not differ strongly from the corresponding estimates for the earlier period (table 2). (Note that the experts forecast the frequency of red tide only through the period 41-100 years from 2011. The simulations that extended to 150 years from 2011 applied the frequencies from years 41-100 to years 101-150.) The median estimate of the frequency of red-tide mortality events was much greater in the SW region than in the other regions, continuing the historical pattern (table 2).

The relative magnitude of manatee mortality events owing to red tide in two regions (NW, AT) was elicited as a ratio relative to the estimated mortality effects in the SW region. A magnitude less than 1 indicates that the experts anticipated a lower additional mortality rate during red-tide years in that region than in the SW region. The median estimates of relative mortality were less than 1 , indicating that red-tide effects will not be as strong in the NW and AT regions as in the SW region (table 3 ). The experts expect additional mortality in red-tide years to be greater in the AT than in the NW. In all cases the uncertainty was large; the 80-percent credible intervals for all parameters allowed for the possibility that the magnitude of red-tide morality in the AT and NW could be much greater or much less than in the SW.

\section{Frequency and Magnitude of Cold-Related Mortality}

On the basis of daily air temperature data during winter for 1950-2013, thresholds were established for classifying years as normal, cold, or severely cold. In the NW region, using the Inverness weather station, the 5-percent quantile of tdef 14 was $336.1 \mathrm{C}$-day, and the 20-percent quantile was 253.8 C-day. Thus, winters with greater than $336.1 \mathrm{C}$-day were classified as severe $(1978,2003,2010)$, and winters with C-day between 253.8 and 336.1 C-day were classified as cold (1958, 1977, 1981, 1985, 1986, 1996, 1998, 2001, 2011). In the USJ region, using the Deland weather station, the threshold for severe cold was 307.6 C-day (1977, 1978, and 2010), and the threshold for cold was 210.9 C-day $(1951,1955$, 1958, 1969, 1981, 1996, 2001, 2009, and 2011). In the AT region, using the Melbourne weather station, the threshold for severe cold was 170.6 C-day (1958, 1978, and 2010), and the threshold for cold was 114.8 C-day $(1969,1970,1971,1977$, 1981, 1996, 2001, 2003, and 2011). In the SW region, two weather stations were used, Ft. Myers and Tampa; their tdef 14 values were standardized to mean 0 and standard deviation 1 , then averaged, and the quantile was determined from the average standard value. The severely cold years were 1958, 1978, and 2010, and the cold years were 1951, 1960, 1964, $1969,1970,1977,1981,1996$, and 2001. For the simulations 

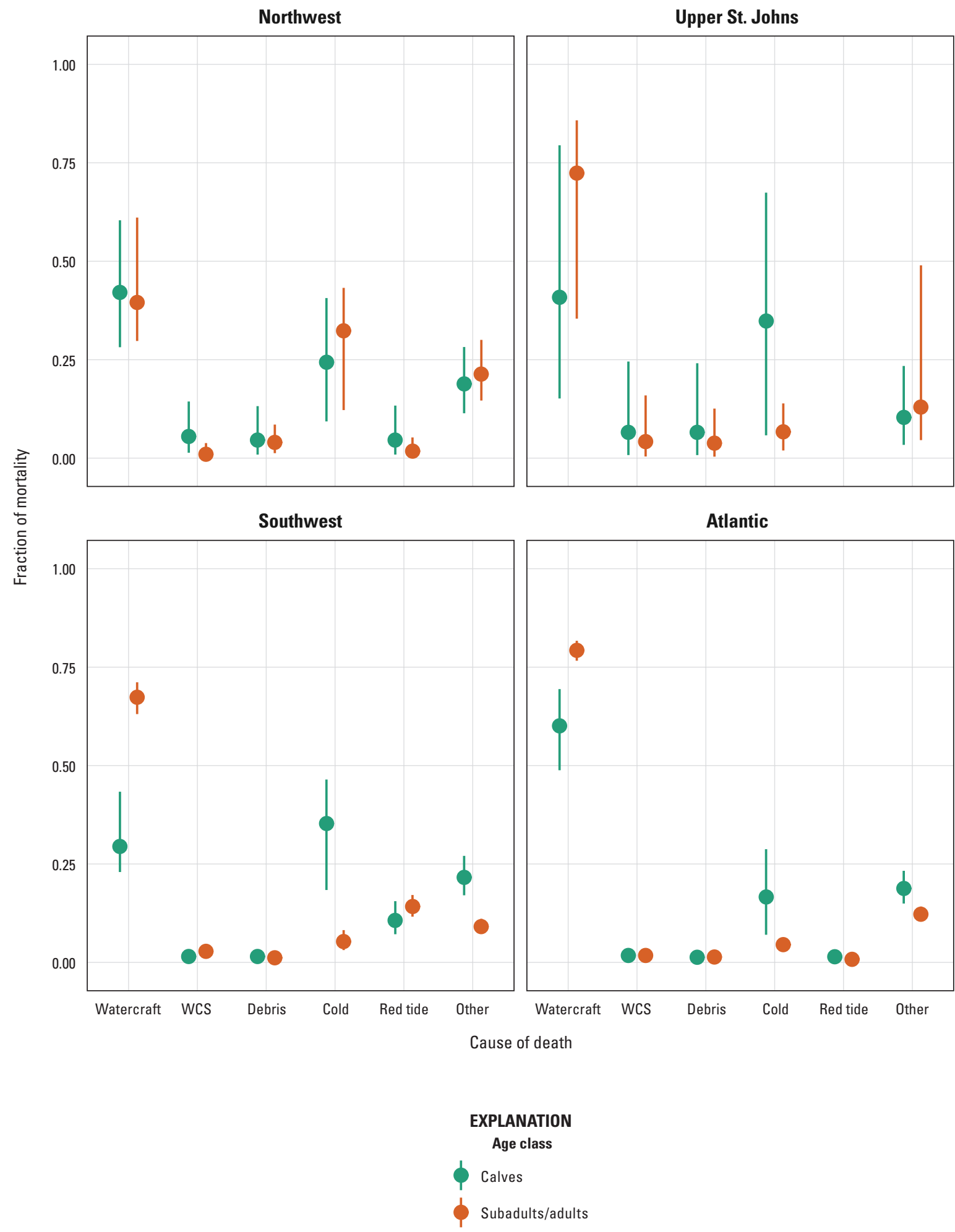

Figure 4. Baseline fractions of mortality of the Florida manatee, by cause of death and age class, in four regions of Florida, 1995-2013. The circles show the mean estimates, and the whiskers show the 95-percent credible intervals from the Bayesian posterior distribution for each parameter. (WCS, water-control structure) 


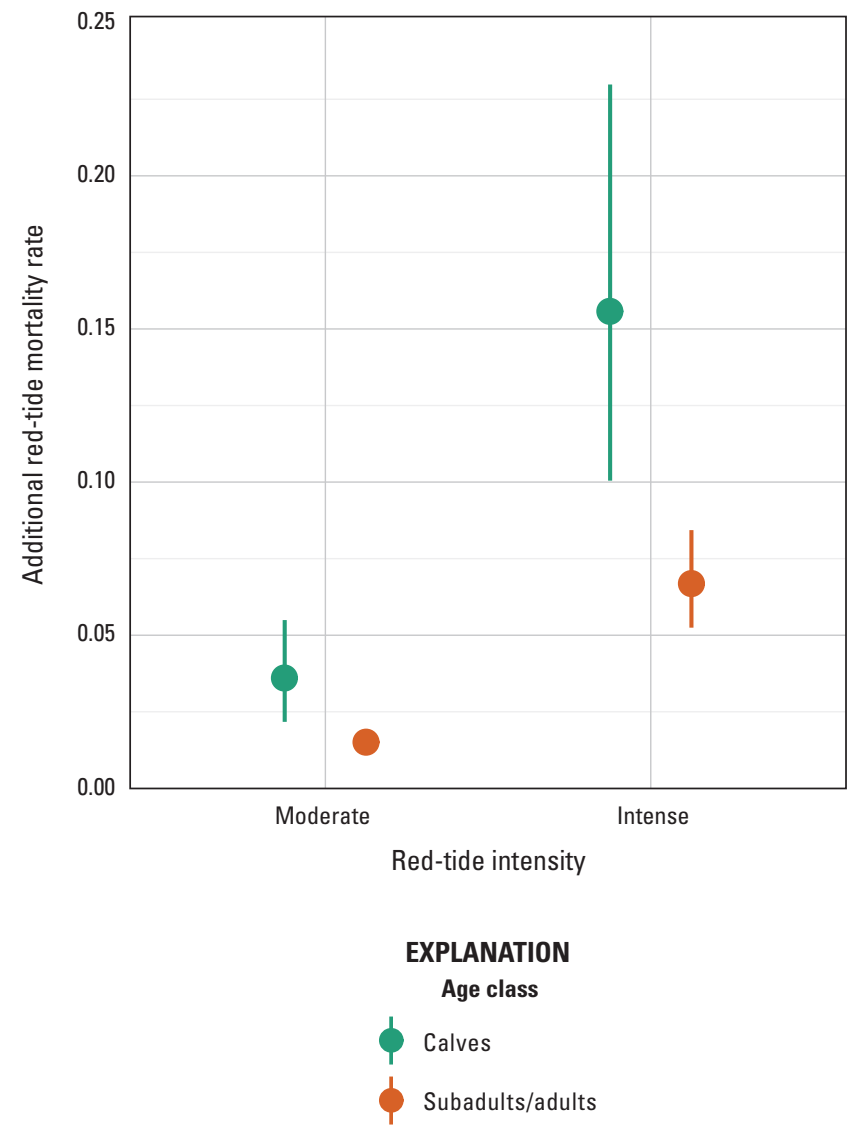

Figure 5. Estimated additional red-tide mortality rate for Florida manatees in the Southwest region of Florida, by intensity and age class, from the hierarchical analysis of manatee carcass data, 1995-2013. The circles show the mean estimates, and the whiskers show the 95-percent credible intervals from the Bayesian posterior distribution for each parameter.

using historical frequencies of cold, the rates of 5 percent and 15 percent were used for severely cold and cold years, respectively.

The period 2001-11 appears to have an abnormally high number of cold years. Across regions and years during that period, 5 of the 44 region-years (11.4 percent) were severely cold, and 9 of the 44 region-years (20.5 percent) were cold. We used these elevated frequencies for the scenarios that assumed cold years would be more frequent in the future.

The estimated rates of additional cold mortality ranged from 0.00 to 0.56 and generally followed the expected patterns: calves $>$ subadults $>$ adults; severe winters $>$ cold $>$ normal; and low habitat quality $>$ medium $>$ high (fig. 6). The exceptions were calves in normal winters and subadults in severe winters, for which the estimates of additional mortality in low-quality habitat were less than those in medium-quality habitat.

\section{Carrying Capacity}

On the basis of the aggregated results from the expert panel, the median estimate of current statewide capacity at all high-quality sites is 5,309 individuals (80-percent credible interval [CI], 2,426-12,464). The median estimate of current capacity at medium-quality sites is 10,122 individuals (80-percent CI, 6,528-17,345), and the median estimate of high- and mediumquality sites combined is 16,363 (80-percent CI, 10,71927,378). The median estimate of current carrying capacity at high- and medium-quality sites combined is 3,187 in the NW region (80-percent CI, 1,118-9,900), 1,457 in the USJ region (80-percent CI, 651-3,668), 4,845 in the AT region (80-percent CI, 2,236-10,990), and 4,923 in the SW region (80-percent CI, $2,775-8,310)$. Over the next century (2010-2110), a significant portion of this capacity is expected to be lost through retirement of industrial facilities and reduction in spring flow (fig. 7); the median estimate of long-term statewide carrying capacity for high- and medium-quality sites combined was 7,146 (80-percent CI, 4,020-14,660). Statewide, the median long-term loss of capacity is expected to be 18.6 percent (80-percent CI, 10.130.3 percent) at high-quality sites and 75.5 percent ( 80 -percent CI, 49.8-88.8 percent) at medium-quality sites. These declines are expected to be greatest in the SW and AT regions owing to the number of power plants operating with once-through cooling in those regions (fig. 7).

\section{Baseline Projections}

The baseline scenario forecasts the trajectory of the Florida manatee population in each of the four regions while accounting for uncertainty. In our judgment, it incorporates the best available scientific and commercial information about Florida manatees and the threats they face.

\section{Population Projections}

Under the baseline scenario, the total statewide population is expected to increase slowly, nearly doubling over 50 years, and then stabilize as it reaches a statewide carrying capacity (fig. 8A). The adult population shows a similar pattern (fig. 8B). Focusing on the median projections, little decline is seen in the long term largely because the loss of warm-water capacity is expected to occur before the population reaches the long-term carrying capacity. There is considerable uncertainty in the future projections of population size, as shown by the wide 95-percent projection intervals. This uncertainty arises from uncertainty about the underlying demographic parameters that drive this population, uncertainty about the current and future warm-water capacity, and chance future events. Nevertheless, the model predicts that it is unlikely $(<2.5$-percent chance) the statewide population will fall below 4,000 individuals over the next 100 years $(2011-2110)$.

The patterns of the population forecast are similar at the coastal scale and at the statewide scale (fig. 9). On the Gulf coast (fig. 9A) and the East coast (fig. 9B), the breeding adult 
Table 2. Estimated historical, 1996-2015, and forecast frequencies of Florida manatee red-tide mortality events in four regions of Florida for simulation years $0-40$.

[Three classes of red-tide mortality years are considered: background, moderate, and intense. The table shows the medians and 80-percent credible intervals for regional probabilities from the appropriate beta or Dirichlet distributions. The historical estimates show the frequency of red-tide mortality events in each region, 1996-2015. Forecasts of future frequency of red-tide mortality events were elicited from an expert panel under two hypotheses: one hypothesis, there are only 2 classes of years (background and red-tide years); and the second hypothesis, there are 3 classes of years (background, moderate, and intense). For the historical and 3-class estimates, the "Moderate or Intense" column shows the sum of the moderate and intense frequencies. The baseline scenario uses a weighted average of the 2- and 3-class frequencies. The model allows the frequencies of red tide to be different in years $0-40$ than in years $41-150$ of the simulations; this table shows the frequency for years 0-40. In most cases, the expert-elicited frequencies for the later period did not differ strongly from the corresponding estimates for the earlier period. --, not applicable]

\begin{tabular}{|c|c|c|c|}
\hline Region & Estimate & $\begin{array}{l}\text { Median frequency } \\
\text { of intense red-tide mortality } \\
\text { (credible interval) }\end{array}$ & $\begin{array}{l}\text { Median frequency of } \\
\text { moderate or intense } \\
\text { red-tide mortality } \\
\text { (credible interval) }\end{array}$ \\
\hline \multirow[b]{2}{*}{ Atlantic } & Historical & 0.000 & $0.050(0.012-0.081)$ \\
\hline & 2-class forecast & -- & $0.065(0.016-0.166)$ \\
\hline \multirow{3}{*}{ Upper St. Johns } & Historical & -- & -- \\
\hline & 2-class forecast & -- & -- \\
\hline & 3-class forecast & -- & -- \\
\hline Northwest & Historical & 0.000 & 0.000 \\
\hline \multirow[t]{2}{*}{ Southwest } & 2-class forecast & -- & $0.380(0.188-0.603)$ \\
\hline & 3-class forecast & $0.154(0.068-0.280)$ & $0.503(0.356-0.649)$ \\
\hline
\end{tabular}

Table 3. Estimated magnitude of Florida manatee red-tide mortality events in two regions of Florida relative to mortality events in the Southwest region for simulation years $0-40$.

[During a moderate or intense red-tide year, the magnitude of manatee red-tide mortality in the Atlantic and Northwest regions is expressed as a multiple of the red-tide mortality in the Southwest region. These multipliers on the magnitude of red tide were estimated by an expert panel because an empirical estimate of red-tide mortality is not yet available outside the Southwest region. The table shows the medians and 80-percent credible intervals from the appropriate lognormal distributions. The baseline scenario uses a weighted average of the 2- and 3-class magnitudes. This table shows the estimates for years $0-40$ of the simulation. In most cases, the expert-elicited frequencies for years 41-150 did not differ strongly from the estimates shown. --, not applicable]

\begin{tabular}{cccc}
\hline Region & Estimate & Intense & Moderate \\
\hline \multirow{4}{*}{ Atlantic } & $\begin{array}{c}\text { 2-class } \\
\text { forecast }\end{array}$ & -- & $0.545(0.074-4.039)$ \\
& $\begin{array}{c}\text { 3-class } \\
\text { forecast }\end{array}$ & $0.647(0.162-2.583)$ & $0.575(0.209-1.579)$ \\
& $\begin{array}{c}\text { 2-class } \\
\text { forecast }\end{array}$ & -- & $0.247(0.039-1.567)$ \\
Northwest & $\begin{array}{c}\text { 3-class } \\
\text { forecast }\end{array}$ & $0.388(0.128-1.173)$ & $0.261(0.063-1.090)$ \\
& &
\end{tabular}

populations are expected to increase over about 50 years, then stabilize. On the East coast, a slight decline in the median projection is evident over the second 50 years as the losses of warm-water capacity in the AT region outpace the gains in the USJ region (fig. 9B).

At the regional level, the baseline forecast reveals a substantial shift in the distribution of adult manatees with the NW and USJ regions showing large projected increases and the SW and AT regions showing moderate long-term decreases after initially increasing (fig. 10). In the NW and USJ regions, the expert panel estimated that there is substantially more carrying capacity for manatees than is currently used, and although this capacity is expected to decrease owing to loss of spring flow, most of that loss is expected to occur before the manatee population increases enough to reach capacity; thus, no observed decline is expected in the median projections (fig. 10). In the SW and AT regions, however, the long-term capacity is not estimated to be substantially larger than the current population sizes because substantial loss of capacity is expected through the loss of industrial warm-water discharges (fig. 7). Thus, the populations are expected to increase slightly over the next few decades but then show long-term decline with the loss of warm-water capacity (fig. 10). 

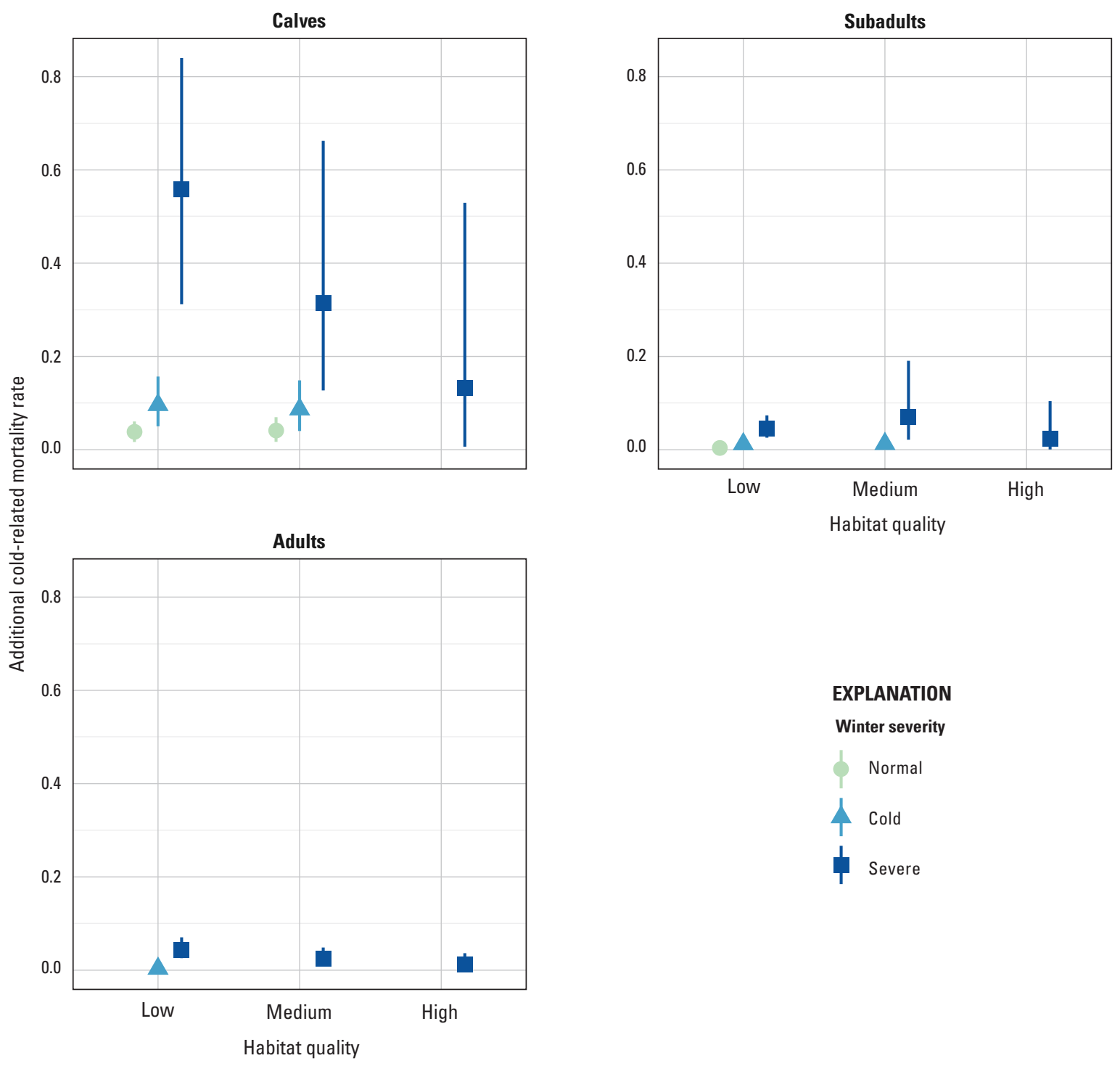

EXPLANATION

Winter severity

Normal

4 Cold

1 Severe

Figure 6. Estimated additional cold-related mortality rate for Florida manatees, by habitat quality, age class, and winter severity, in all regions of Florida combined, 1995-2013. The symbols show the mean estimates, and the whiskers show the 95-percent credible intervals from the Bayesian posterior distribution for each parameter. Missing estimates (for example, additional mortality of calves during a normal or cold year in high-quality habitat) are those that are assumed to be part of the baseline mortality (that is, the additional mortality is 0 ).

\section{Quasi-Extinction Probability}

The probability that the adult population on a coast will fall below a given threshold is an increasing function of the threshold (fig. 11). For instance, the probability that the Gulf coast population will fall below a threshold within 100 years increases from 0.14 percent for a threshold of 500 adults to 2.54 percent for a threshold of 1,000 adults (fig. 11A). The probability of quasi-extinction is also a function of the time frame. For example, the probability that the East coast population will fall below 500 adults increases from 0.28 percent in 100 years to 0.46 percent in 150 years (fig. $11 B$ ). Perhaps the most relevant metric is the probability of the adult population falling below a particular threshold on either the Gulf coast or the East coast (see "Baseline Status" in the "Discussion" section). The probability of the adult population falling below 500 animals on either coast within 100 years is 0.42 percent (fig. $11 C$, table 4). The baseline probabilities of quasi-extinction at 50,100, and 150 years for thresholds of 100,250 , and 500 adults on either coast are shown in table 4. 

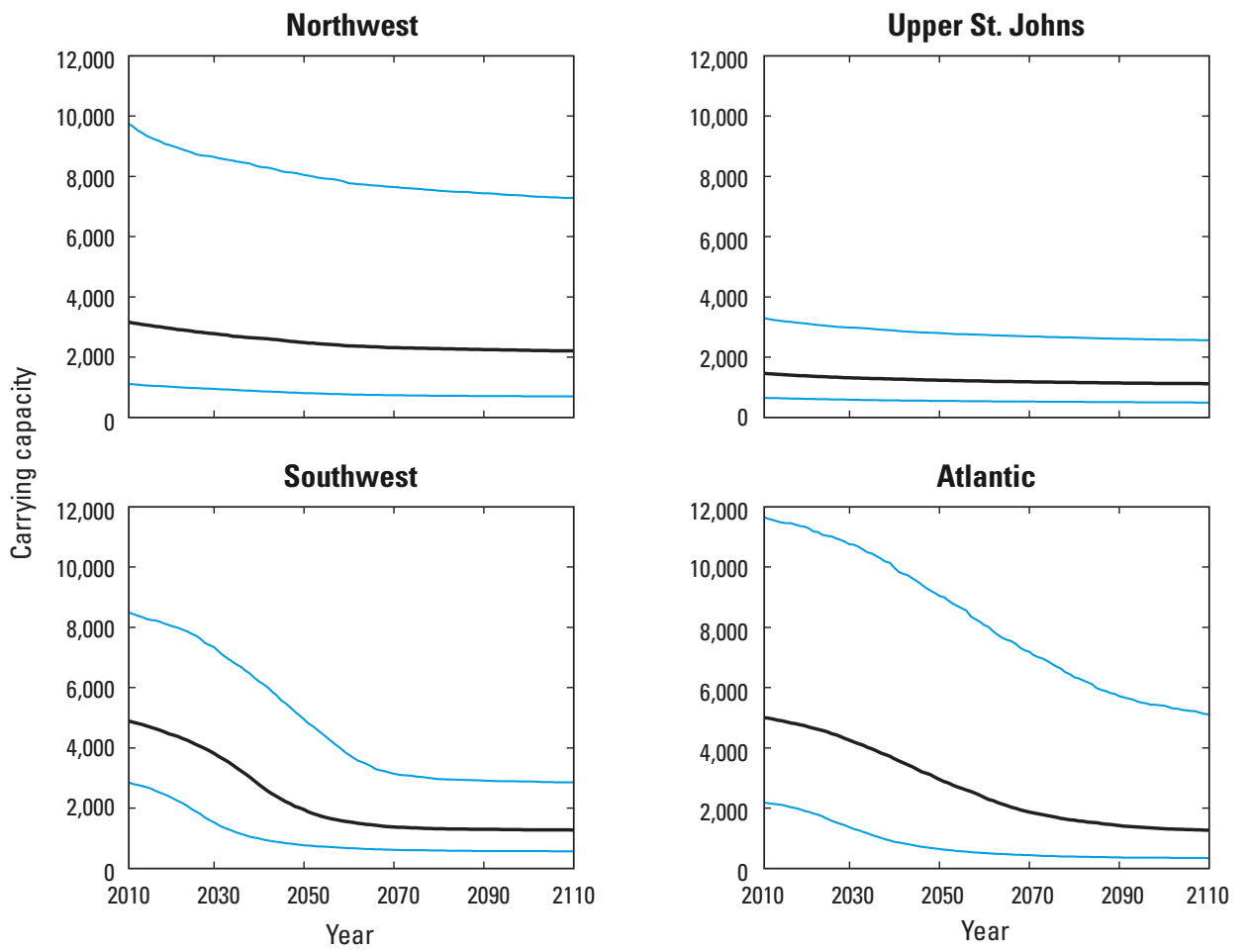

\section{EXPLANATION}

80-percent credible intervals

Median estimate

Figure 7. Estimated regional carrying capacity for Florida manatees in four regions of Florida, 2011-2110. The estimates incorporate the anticipated loss of industrial effluents and reduction in spring flow. The 80-percent credible intervals reflect uncertainty in the capacity. The estimates and their uncertainty were determined using an expert-panel process informed by site-based empirical estimates (M.C. Runge, USGS and C.J. Deutsch, FWRI, written commun., 2017).

\section{Expected Minimum Population Size}

Over the 5,000 replicates of the baseline scenario, the minimum total population size on either the Gulf coast or the East coast over the first 100 years ranged from about 200 to about 3,800 (fig. 12). The EMP, that is the average of these minimum values, is 2,361 . The EMP is a decreasing function of the time frame; the EMP is 2,555 over 50 years, 2,361 over 100 years, and 2,248 over 150 years (table 4 ).

\section{Probability of Population Decline}

Statewide, the probabilities that the adult manatee population size will decline by 10,30 , or 50 percent in 100 years are low $(4.9,1.5$, and 0.1 percent, respectively; table 5). At the coastal scale, the probabilities are higher, especially on the East coast, where the high risk of decline in the AT region (49.9-percent chance of a 30-percent decline in 100 years) is greater than the negligible risk of decline in the smaller USJ region. The probabilities of decline in the SW region are similar to those in the AT region (table 5). Focusing on the risk of decline on either coast, there is a fairly high probability (28.1 percent) of a 10-percent decline in 100 years, a moderate probability ( 15.9 percent) of a 30 -percent decline, and a small but not negligible risk (5.2 percent) of a 50 -percent decline (table 5).

Some of the IUCN criteria focus on the probability of decline over three generations (which we assume to be 60 years; Haubold and others, 2006). The probabilities of the adult manatee population declining statewide by 10,30 , or 50 percent within 60 years are $1.48,0.26$, and 0.02 percent, respectively. The corresponding probabilities of the adult manatee population declining by 10,30 , or 50 percent on either coast within 60 years are $15.5,6.6$, and 1.8 percent, respectively. 

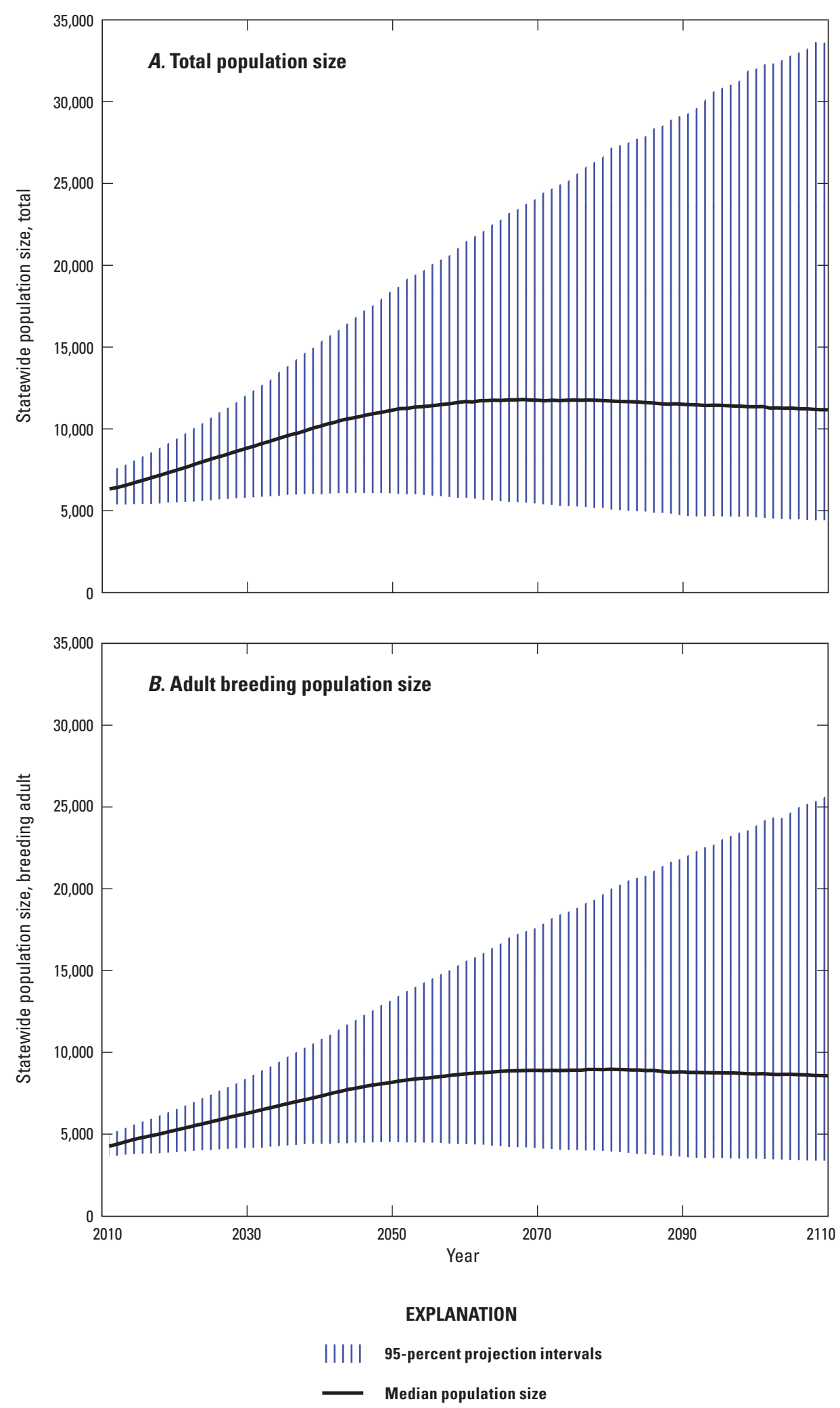

Figure 8. Estimated Florida manatee population size under the baseline scenario, 2011-2110, for $A$, the total population size and $B$, the breeding adult population size in all four regions of Florida combined. 


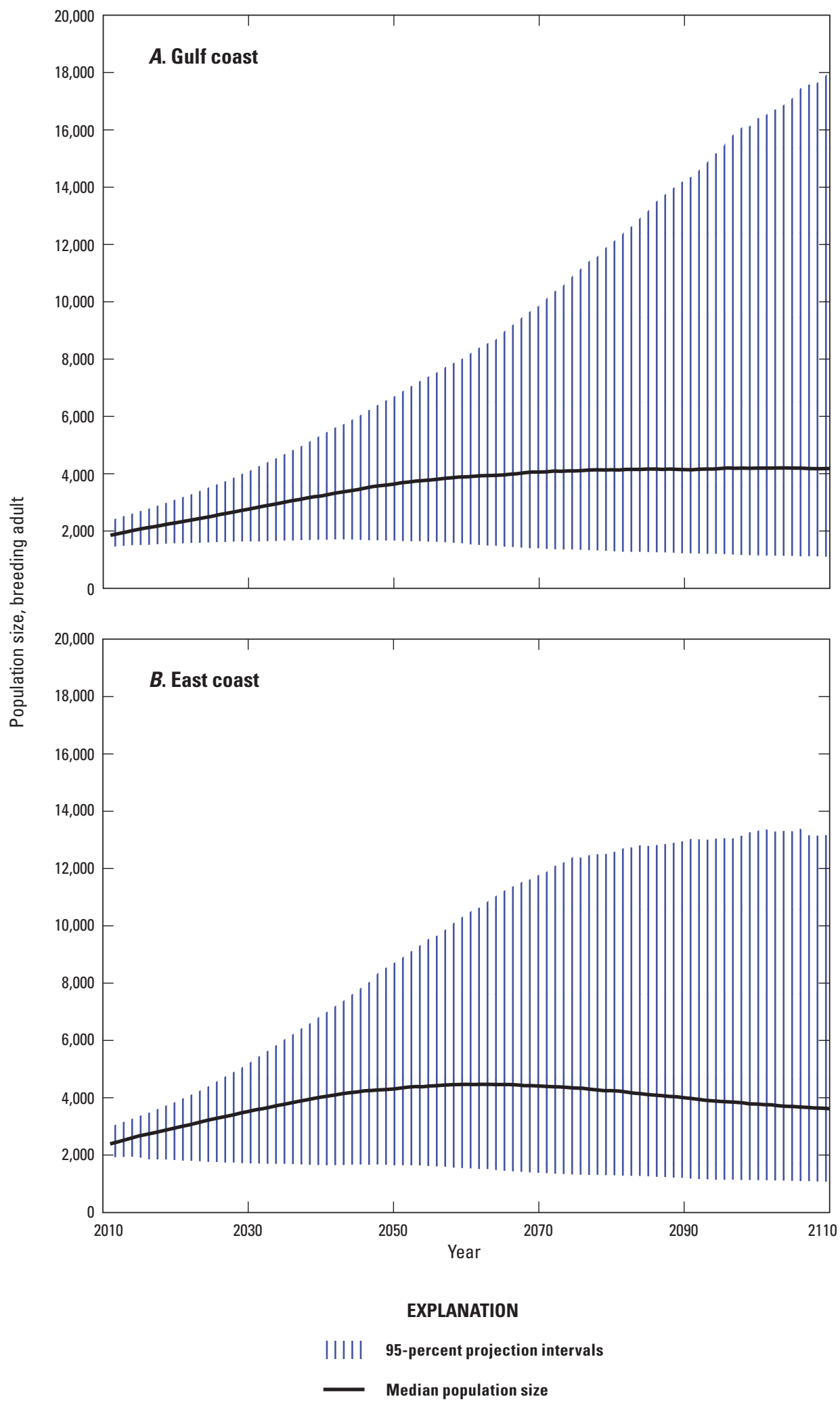

Figure 9. Estimated Florida manatee breeding adult population size under the baseline scenario, 2011-2110, for $A$, the Gulf coast and $B$, the East coast of Florida. 

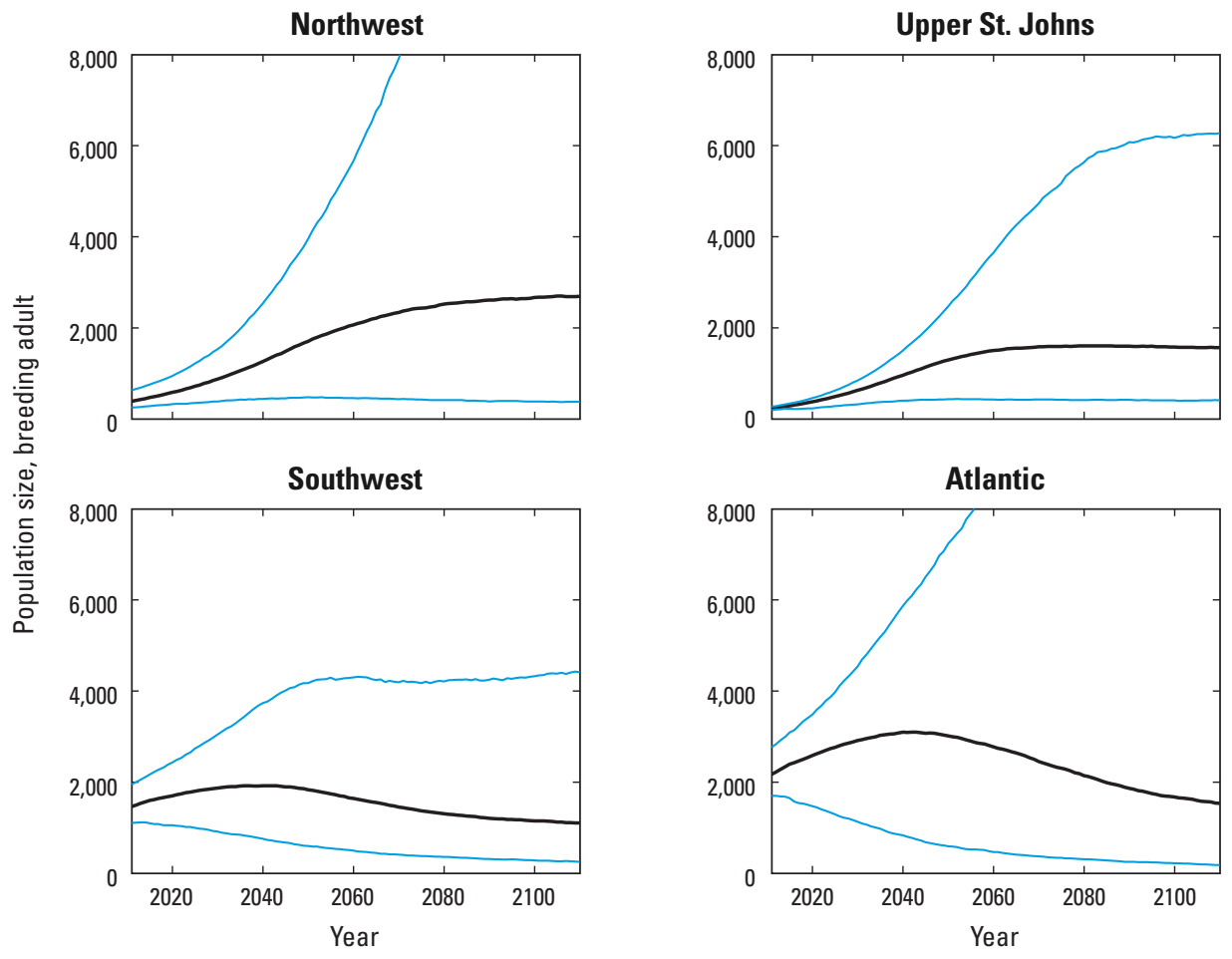

EXPLANATION

95-percent projection intervals

Median population size

Figure 10. Estimated Florida manatee breeding adult population size under the baseline scenario in four regions of Florida, 2011-2110.

\section{Threats Analysis}

To assess the strength of different threats, those threats were removed one at a time, and the results were compared to the baseline (table 4, fig. 13). The threats fall into two groups: those that have little effect on the coastal quasi-extinction probability or EMP (water-control structures, marine debris) and those that have a pronounced effect (watercraft, warmwater loss, red tide) (fig. 13). Red-tide related mortality is the strongest threat as measured by the probability of quasiextinction; removing the red-tide threat alone reduces the probability of the adult population dropping below 500 individuals on either coast in 150 years from 0.98 percent (baseline) to 0.22 percent (table 4 ). Watercraft-related mortality and warm-water loss, however, are the strongest threats as measured by the EMP; removing the threat of warm-water loss, for instance, raises the EMP over 150 years from 2,248 under the baseline to 2,602 (table 4). The three strongest threats exert risk at different time frames. Red-tide mortality is the most immediate threat; at 50 years, it produces the strongest effect on the risk of quasi-extinction of the threats considered (table 4). Removing the threat of warm-water loss, however, does not have a discernible effect on the probability of quasi-extinction at 50 years because the onset of the threat is delayed, whereas warm-water loss does have a strong effect at 100 and 150 years (table 4). The timing of the watercraft threat is intermediate between red tide and warm-water loss.

For practical reasons, full removal of any threat is likely to be impossible, and indeed, threats could potentially strengthen. Thus, it is useful to forecast the effects of partial removal or increase of threats. For the threat owing to watercraft-related mortality, the probability of falling below 500 adults on either coast within 100 years increases roughly linearly from 0.18 percent to 0.42 percent as the level of the threat increases from 0 to baseline (fig. 14). But as the threat increases to levels higher than baseline, the risk of quasiextinction increases exponentially, rising to nearly 4 percent when the mortality rate owing to watercraft doubles (fig. 14).

For the threat owing to loss of warm-water habitat, the probability of falling below 500 adults on either coast within 100 years increases from 0.26 percent to 0.42 percent as the level of the threat increases from 0 to baseline; most of that increase occurs between a threat level of 0.8 and 1.0 (fig. 15). That is, most of the benefit of removing the warm-water threat comes from removing just 20 percent of it. Accelerating the threat increases the risk of quasi-extinction but not 


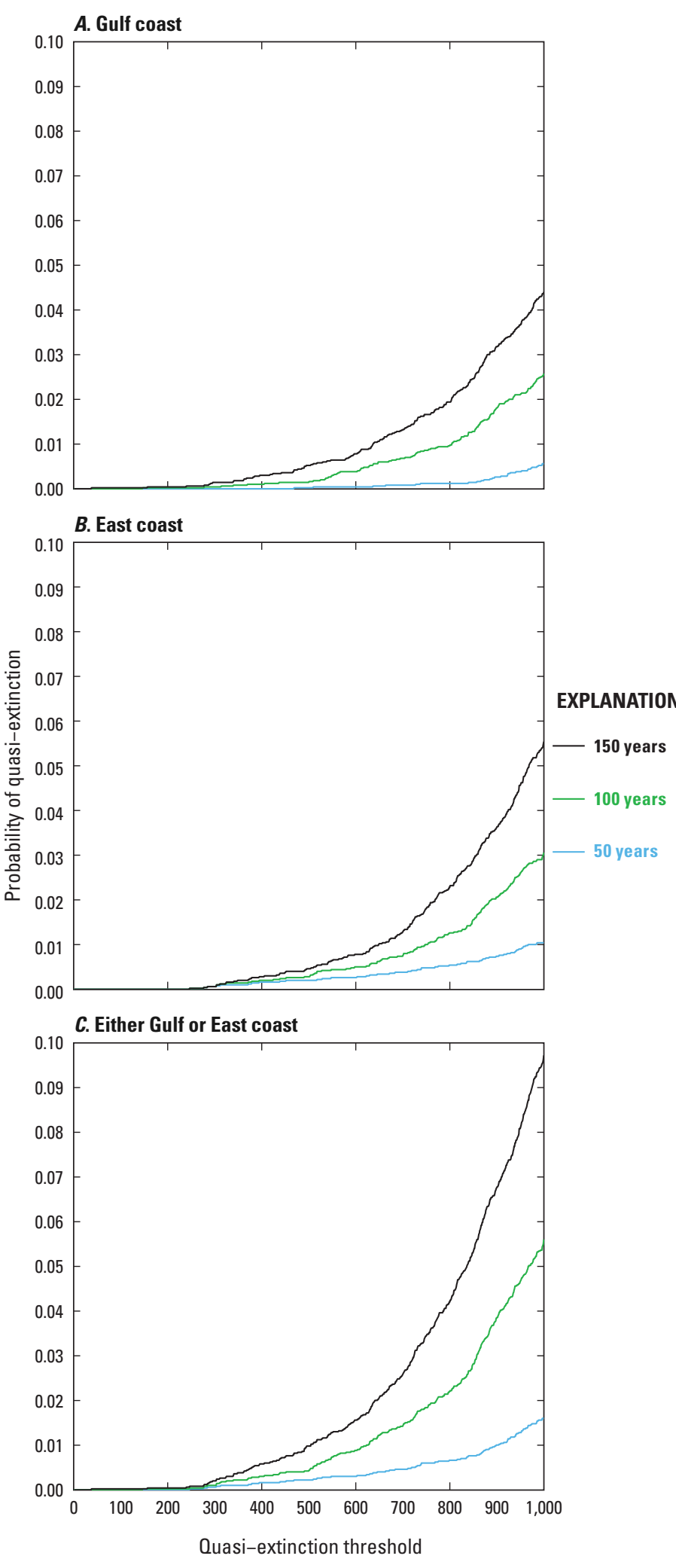

Figure 11. Probability of the adult population of Florida manatees falling below a quasi-extinction threshold within 50,100 , or 150 years from 2011, as a function of the quasi-extinction threshold under the baseline scenario on $A$, the Gulf coast, $B$, the East coast, or $C$, either coast of Florida.

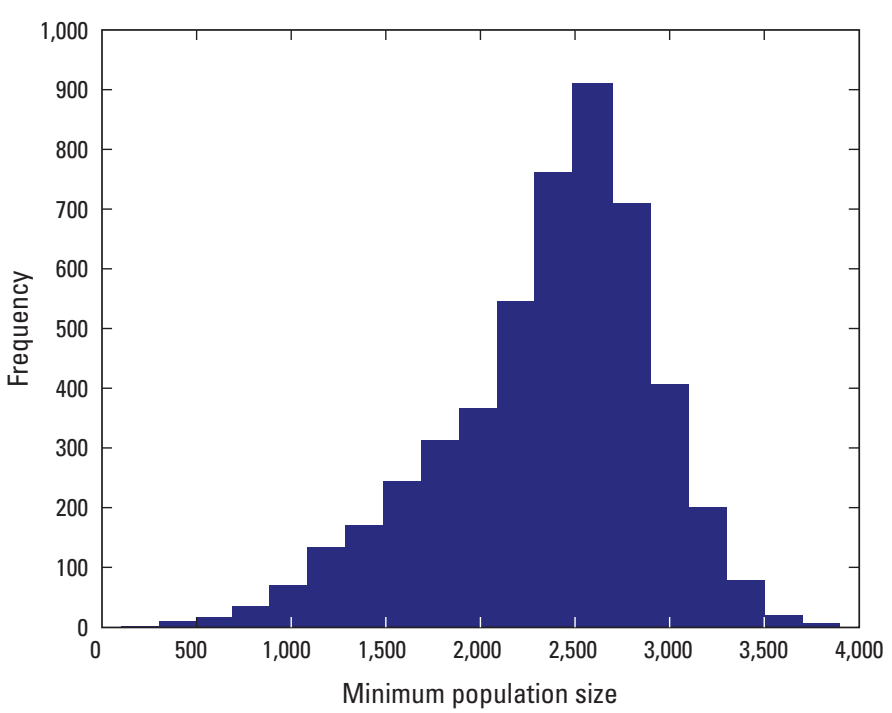

Figure 12. Minimum total population size of Florida manatees on either the Gulf or East coast of Florida over the period 2011-2110.

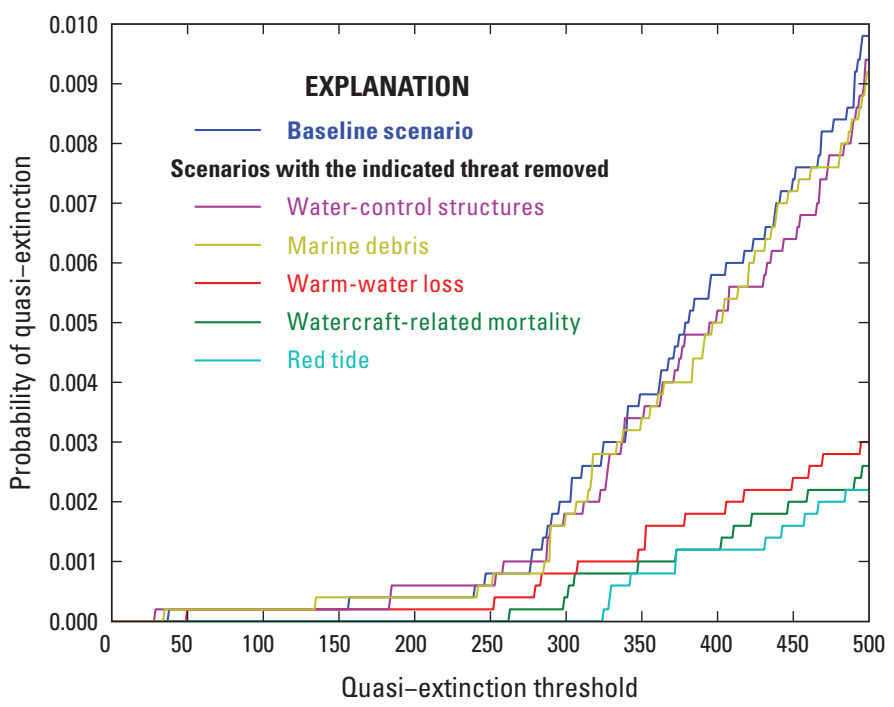

Figure 13. Probability of the adult population of Florida manatees falling below a quasi-extinction threshold on either the Gulf or the East coast of Florida within 150 years from 2011 for the baseline scenario and five scenarios with the indicated threat removed.

substantially. With immediate loss of coal-fired power plants and the warm-water effluent they produce, the risk of quasiextinction rises to 0.46 percent; with immediate loss of all industrial warm water, the risk rises to 0.72 percent (fig. 15). If the threat of watercraft-related mortality is removed, the effect of the threat of warm-water loss on quasi-extinction becomes negligible (fig. 15).

Combinations of partial removal of the threats owing to watercraft and warm-water loss were evaluated, as shown in the contour plot in figure 16. The baseline conditions occur in the top right corner (probability of falling below 500 adults on either coast within 150 years is 0.98 percent); full removal 
Table 4. Probability of the adult Florida manatee population falling below a quasi-extinction threshold of 100, 250, or 500 animals on either the Gulf coast or the East coast of Florida in 50, 100, or 150 years from 2011 with threat removal scenarios.

[The scenarios consider the removal of threats one at a time. For example, in the absence of the threat from moderate or severe red tide (-Red tide), the probability is 0.22 percent that the adult population will fall below 500 animals on either the East or Gulf coasts within 150 years, and the probability is 0.98 percent with the threat at its current level (baseline). Because only 5,000 replicates were run, where the estimate is 0 , this should be understood as less than 0.0002 (less than 0.02 -percent probability of quasi-extinction). The expected minimum population (EMP) size for each scenario is calculated by finding the minimum total population size of each simulated replicate on either coast over the specified timeframe, then taking the mean value over all replicates. -, indicates threat is removed]

\begin{tabular}{|c|c|c|c|c|c|}
\hline Scenario & Years & \multicolumn{3}{|c|}{ Quasi-extinction threshold } & EMP \\
\hline Baseline & 50 & 0.0000 & 0.0002 & 0.0022 & 2,555 \\
\hline -Watercraft & 50 & 0.0000 & 0.0000 & 0.0014 & 2,707 \\
\hline -Warm-water loss & 50 & 0.0000 & 0.0000 & 0.0024 & 2,630 \\
\hline -Water control structures & 50 & 0.0000 & 0.0000 & 0.0022 & 2,564 \\
\hline -Marine debris & 50 & 0.0000 & 0.0000 & 0.0024 & 2,563 \\
\hline Baseline & 100 & 0.0000 & 0.0004 & 0.0042 & 2,361 \\
\hline -Watercraft & 100 & 0.0000 & 0.0000 & 0.0018 & 2,622 \\
\hline -Water control structures & 100 & 0.0000 & 0.0004 & 0.0044 & 2,373 \\
\hline -Marine debris & 100 & 0.0000 & 0.0002 & 0.0050 & 2,371 \\
\hline Baseline & 150 & 0.0002 & 0.0008 & 0.0098 & 2,248 \\
\hline -Watercraft & 150 & 0.0000 & 0.0000 & 0.0026 & 2,542 \\
\hline -Warm-water loss & 150 & 0.0002 & 0.0002 & 0.0030 & 2,602 \\
\hline -Red tide & 150 & 0.0000 & 0.0000 & 0.0022 & 2,412 \\
\hline -Water control structures & 150 & 0.0002 & 0.0006 & 0.0094 & 2,261 \\
\hline -Marine debris & 150 & 0.0002 & 0.0006 & 0.0092 & 2,259 \\
\hline
\end{tabular}

of both threats occurs in the bottom left corner (probability of quasi-extinction, 0.14 percent). The contour plot reveals that there are multiple management paths to the same outcome. For example, if the desire were to reduce the probability of quasi-extinction to 0.40 percent, this could be achieved in a number of ways: by removing 20 percent of the warm-water threat but not addressing the watercraft threat, by removing about 55 percent of the watercraft threat but not addressing the warm-water threat, or by any combination of the threats that fall along the 0.004 contour line (fig. 16).

\section{Potential Emerging Threats}

The scenarios that represented potential emerging threats take into consideration increases to existing threats, appearance of new threats, and multiple threats increasing at the same time. All results are compared to the baseline scenario.

\section{Frequency of Cold-Related Mortality}

Three of the four hypotheses advanced to explain the cold mortality events of 2009-10 and 2010-11 forecast relatively similar EMPs and risks of quasi-extinction. For hypothesis 1 , the baseline scenario, which uses historical frequencies of cold, the probability of falling below 500 adults on either coast within 100 years is 0.42 percent, and the EMP is 2,361 . If the frequencies of cold and severe cold are elevated to 20.5 and 11.4 percent, respectively, as in hypothesis 4 , the quasiextinction probability rises slightly to 0.58 percent, and the EMP drops to 2,296 (table 6). If we force exposure to backto-back severely cold years at the beginning of each simulated replicate, as in hypothesis 3 , the quasi-extinction probability is 0.72 percent, and the EMP is 1,924 , which are relatively similar to the baseline (table 6). Hypothesis 2, which posits that the back-to-back cold events are an indicator that the regional populations have reached carrying capacity, stands out. Under 
Table 5. Probability of adult Florida manatee population declines of 10,30 , or 50 percent at 100 years from 2011 compared to the 2011 adult population size, by state, coast, and region.

[For each of the 5,000 replicates in the baseline scenario, the adult breeding population size at year 100 was compared to the initial population size, then the fraction of replicates that showed a decline of at least 10,30 , or 50 percent was calculated. The Gulf coast consists of the Northwest and Southwest regions; the East coast consists of the Atlantic and Upper St. Johns regions. The calculation for "either coast" tallies the number of replicates in which either the Gulf coast population or the East coast population declined by the relevant magnitude. $\geq$, greater than or equal to]

\begin{tabular}{lccc}
\hline & \multicolumn{3}{c}{ Probability of decline at 100 years } \\
& $\mathbf{1 0}$-percent & $\mathbf{3}$ 30-percent & $\geq$ 50-percent \\
\hline Statewide & 0.0490 & 0.0150 & 0.0014 \\
Gulf coast & 0.0882 & 0.0440 & 0.0142 \\
East coast & 0.2134 & 0.1192 & 0.0386 \\
Either coast & 0.2808 & 0.1590 & 0.0520 \\
\hline & Regions & & \\
\hline Atlantic & 0.5910 & 0.4988 & 0.3744 \\
Upper St. Johns & 0.0020 & 0.0006 & 0.0002 \\
Northwest & 0.0254 & 0.0170 & 0.0104 \\
Southwest & 0.5946 & 0.4560 & 0.2876 \\
\hline
\end{tabular}

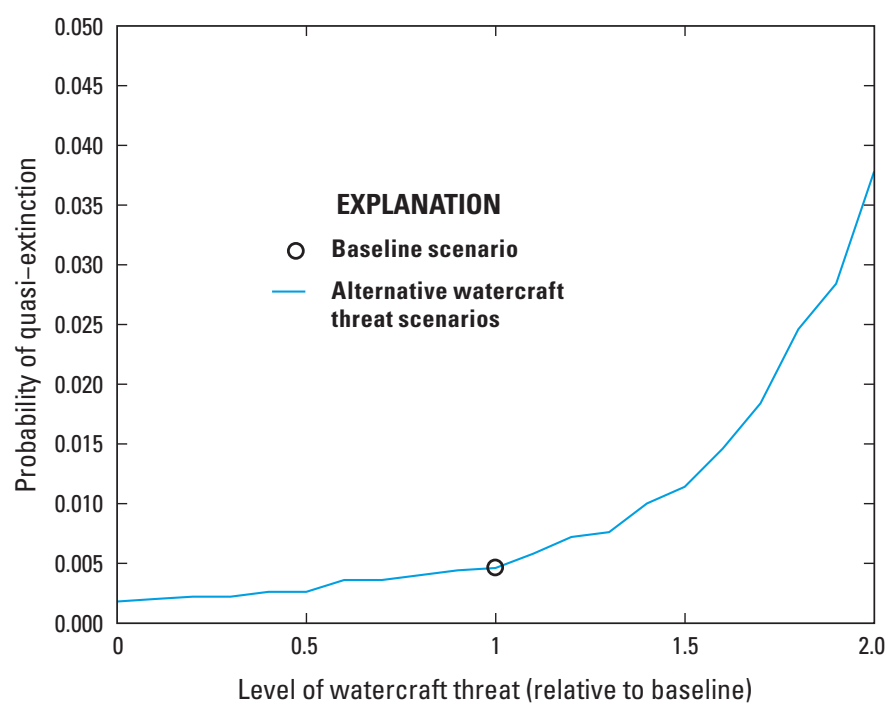

Figure 14. Probability of the adult population of Florida manatees falling below 500 animals (quasi-extinction) on either the Gulf or the East coast of Florida within 100 years from 2011 as a function of watercraft-related mortality. The level of the watercraft threat is expressed on a scale proportional to the baseline level.

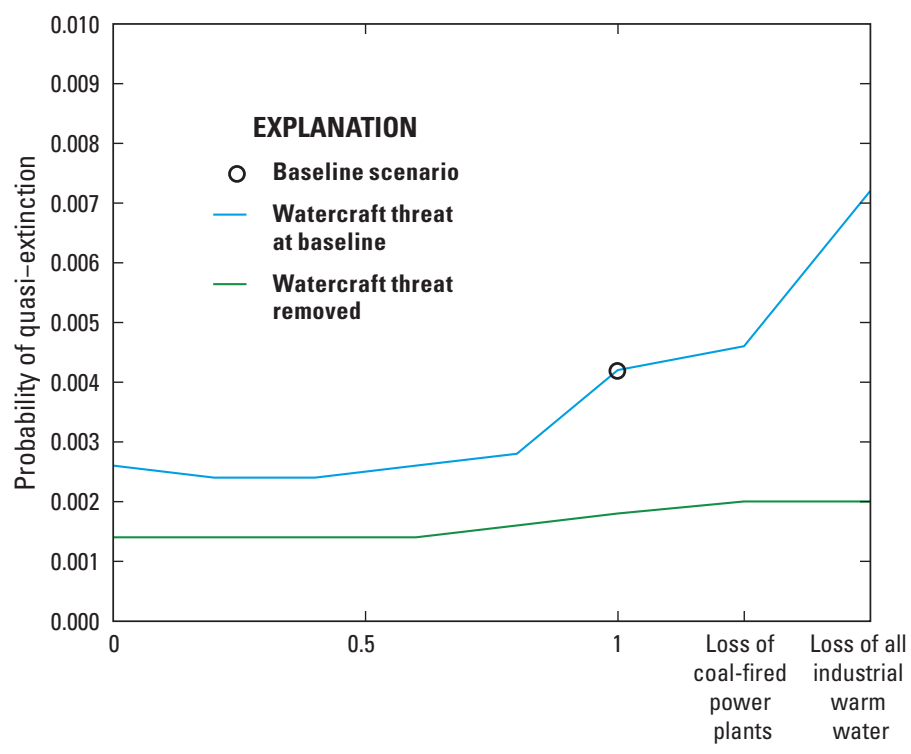

Level of warm-water-loss threat (relative to baseline)

Figure 15. Probability of the adult population of Florida manatees falling below 500 animals (quasi-extinction) on either the Gulf or East coast of Florida within 100 years from 2011 as a function of the level of loss of warm-water capacity with and without removal of the watercraft threat.

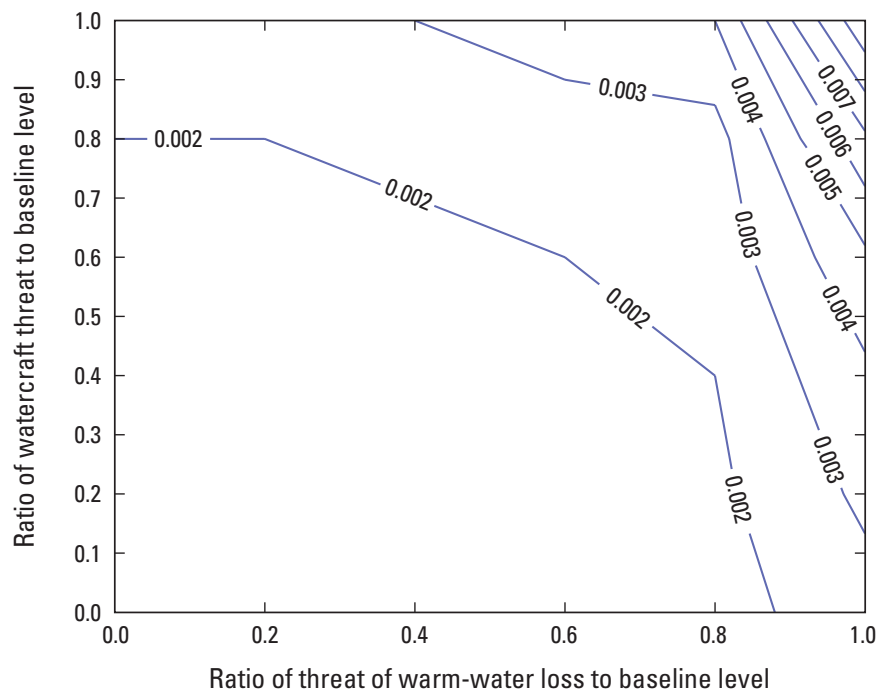

Figure 16. Probability of the adult population of Florida manatees falling below 500 animals on either the Gulf or the East coast of Florida within 150 years from 2011 as a function of the two primary threats, watercraft-related mortality and warm-water loss. The axes are expressed as the ratio of the particular threat level to the baseline level; the contours mark the probabilities of quasiextinction. The baseline conditions occur in the upper-right corner of the graph. 
Table 6. Probability of the adult Florida manatee population on either the Gulf coast or the East coast of Florida falling below 100, 250, or 500 animals in 100 years from 2011 , using the emerging threats scenarios.

[These scenarios consider a series of hypotheses for potential emerging threats. Note that cold-hypothesis 1 is the same as the baseline scenario. Because only 5,000 replicates were run, where the estimate is 0 , this should be understood as less than 0.0002 . The expected minimum population (EMP) size for each scenario is calculated by finding the minimum total population size of each simulated replicate on either coast over 100 years, then taking the mean value over all replicates]

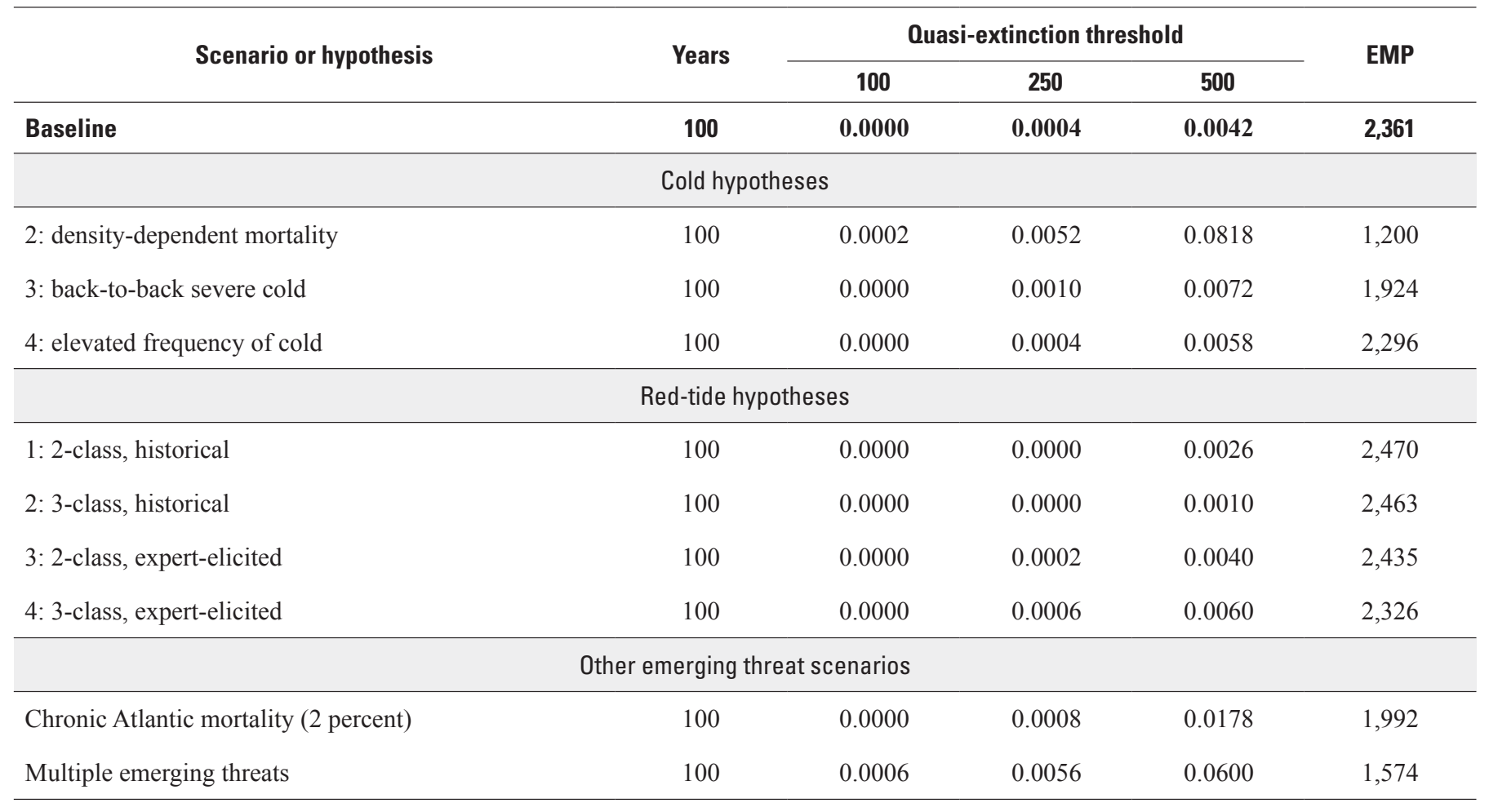

hypothesis 2 , the estimates of the initial statewide carrying capacity (high- and medium-quality combined) change markedly (fig. 17) from a median of 16,363 (80-percent CI, 10,719-27,378) under the baseline to 6,155 (80-percent CI, $5,213-6,917)$ under hypothesis 2 . With such a small initial carrying capacity, the probability of quasi-extinction increases to 8.18 percent, and the EMP drops to 1,200 (table 6).

\section{Frequency of Red Tide Events}

The alternative hypotheses about the future occurrence of red-tide manatee mortality produce small differences in the probability of quasi-extinction and EMP (table 6). The two hypotheses that assume elevated frequencies of red-tide mortality in the future (expert-elicited hypotheses 3 and 4) produce higher probabilities of quasi-extinction and lower EMPs than the hypotheses that assume historical frequencies of red-tide mortality (table 6). The 2-class and 3-class historical hypotheses also differ. For hypothesis 4, the 3-class expert-elicited, elevated frequency hypothesis, the probability of falling below 500 adults on either coast within 100 years is 0.60 percent, and the EMP over 100 years is 2,326 (table 6). Note that the baseline scenario is a weighted mixture of hypotheses 3 and 4, and the quasi-extinction and EMP results of the baseline scenario are intermediate between those of the two component hypotheses.

The effects of the red-tide hypotheses largely arise from effects in the SW region, with a minor contribution from effects in the AT region (fig. 18). Contrasting red-tide hypotheses 1 and 4, the increased frequency and inclusion of three classes of red-tide years raises the probability of quasiextinction considerably in the SW region, somewhat in the AT region, and hardly at all in the NW region. The model has no red-tide effects in the USJ region. Note that the higher probabilities of quasi-extinction for a given threshold shown for the NW and USJ regions are largely a result of their smaller starting population sizes; the probability must be 1.0 when the threshold level exceeds the initial population size.

\section{Chronic Atlantic Mortality}

In this scenario, a chronic annual additional mortality of 2 percent was applied to all stage classes in the AT region, regardless of density. Compared to the baseline scenario, the probability of falling below 500 adults on either coast within 100 years quadrupled, rising from 0.42 percent to 1.78 percent, and the expected minimum population size on either coast over 100 years dropped from 2,361 to 1,992 (table 6). 

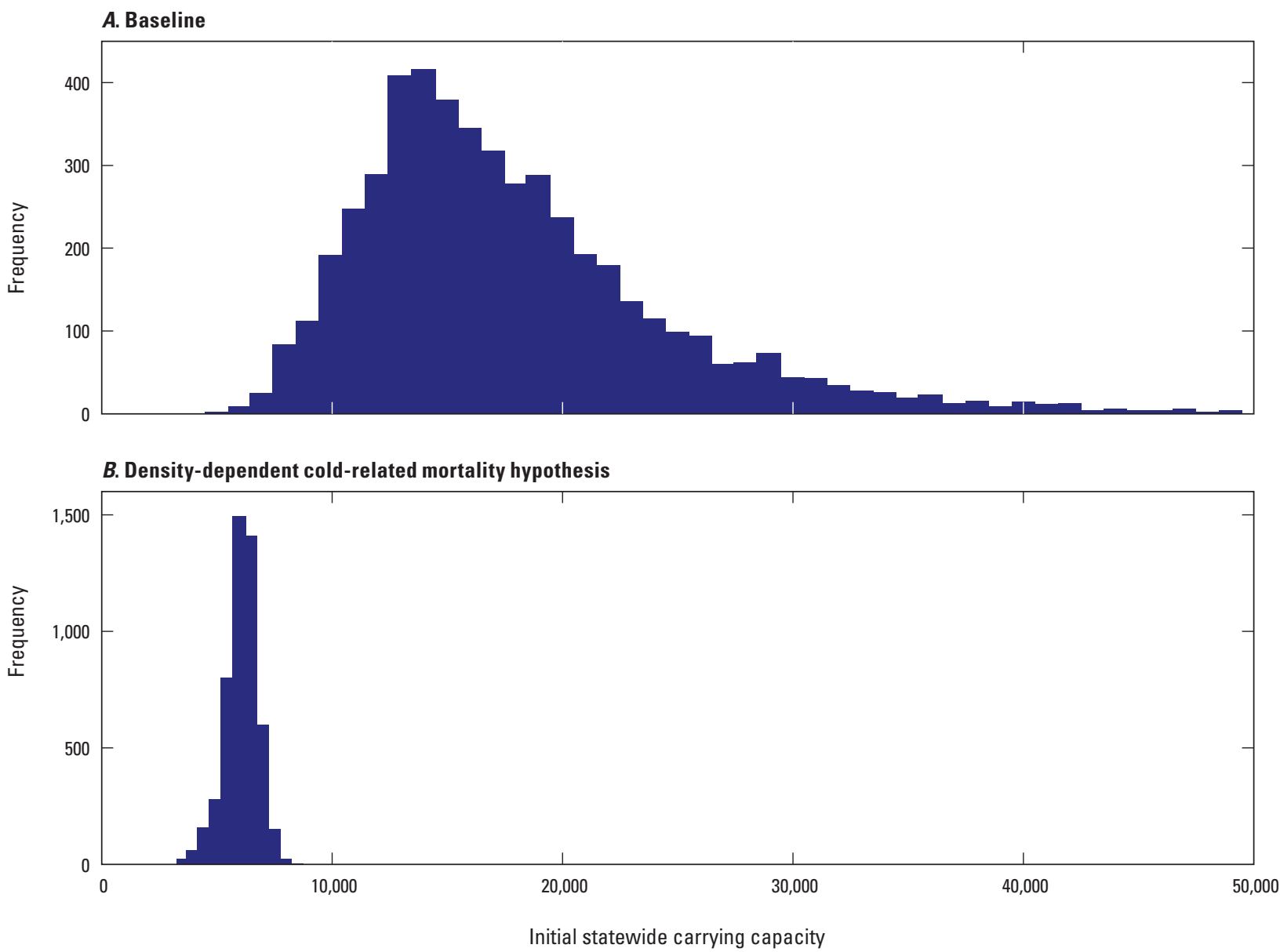

Figure 17. Warm-water carrying capacity for Florida manatees in 2011, high- and medium-quality capacities combined across four regions of Florida, under two hypotheses for the 2009-11 cold-related mortality events: $A$, baseline hypothesis and $B$, density-dependent cold-related mortality hypothesis.

\section{Multiple Emerging Threats in Combination}

In the final emerging threats scenario, many threats were increased in combination (see "Potential Emerging Threats" in the "Methods" section). Compared to the baseline scenario, the probability of falling below 500 adults on either coast within 100 years rose about 14 -fold from 0.42 percent to 6.0 percent, and the expected minimum population size on either coast over 100 years dropped by about one-third from 2,361 to 1,574 (table 6).

\section{Sensitivity Analysis}

The sensitivity of EMP and the risk of quasi-extinction to two elements of the CBM were tested, one representing parametric uncertainty about the long-term loss of carrying capacity at springs and one representing structural uncertainty about the behavioral rules manatees use to distribute among warm-water sites.

\section{Spring Flow}

Uncertainty in the long-term change in warm-water carrying capacity at springs had a small effect on the EMP and probability of quasi-extinction. In the baseline scenario, the mean change in warm-water capacity in high- and mediumquality natural sites was -27 percent. The probability of falling below 500 adults on either coast within 100 years was 0.42 percent, and the expected minimum population size on either coast over 100 years was 2,361. These measures of risk improved as the spring capacity change was set at higher values; the EMP, the more sensitive of the two measures, increased to 2,479 when the spring capacity change improved to +10 percent (table 7 ). In the worst case examined, with 

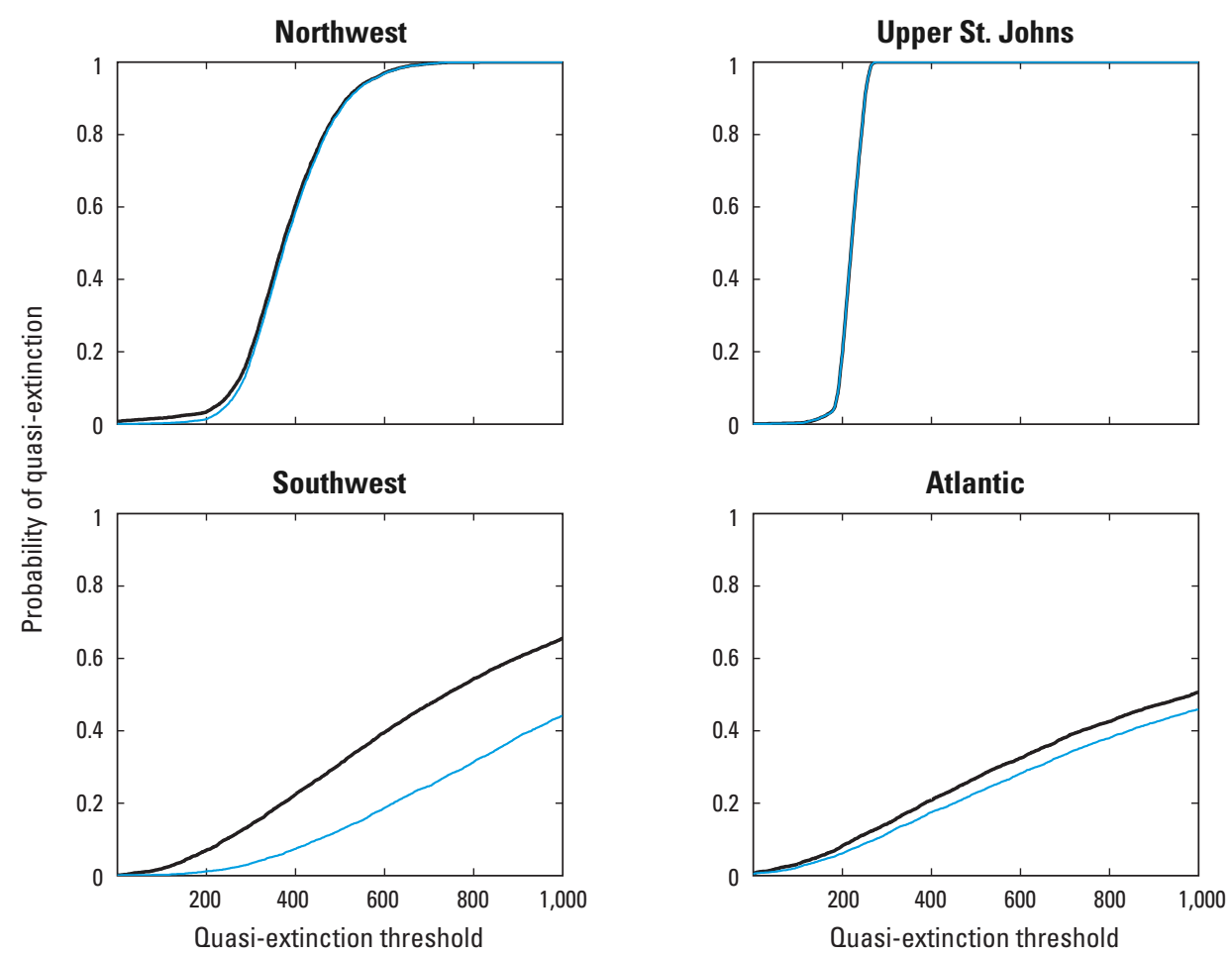

EXPLANATION

Hypothesis 4: elevated frequency and 3 classes of red tide
Hypothesis 1: historical frequency and 2 classes of red tide

Figure 18. Probability of the adult population of Florida manatees falling below a quasi-extinction threshold within 150 years from 2011 as a function of the threshold under two hypotheses about the future frequency of red-tide mortality events in four regions of Florida. For hypothesis 1, red-tide events were assumed to occur in the future with the same frequency as in the past, and two classes of red-tide years were assumed. For hypothesis 4 , an elevated frequency of red tide, as estimated by an expert panel, was assumed in the future, and three classes of red-tide years were assumed. Note that no red-tide mortality is expected to occur in the Upper St. Johns region.

Table 7. Sensitivity of probability of Florida manatee quasi-extinction and expected minimum population size to uncertainty in the longterm change in warm-water carrying capacity provided by springs on the Gulf coast or East coast of Florida.

[The values shown are the probability of the adult population on either the Gulf coast or the East coast falling below the specified threshold at any point in the first 100 years of the simulation. In the scenarios for this sensitivity analysis, the long-term change in carrying capacity at high-quality and medium-quality natural warm-water sites was set at a fixed value, rather than sampling from a distribution (as in the baseline scenario). EMP, expected minimum population size; $\%$, percent]

\begin{tabular}{|c|c|c|c|c|c|}
\hline Scenario & Years & \multicolumn{3}{|c|}{ Quasi-extinction threshold } & EMP \\
\hline Spring capacity $-50 \%$ & 100 & 0.0000 & 0.0002 & 0.0084 & 2,229 \\
\hline Baseline (mean -27\%) & 100 & 0.0000 & 0.0004 & 0.0042 & 2,361 \\
\hline Spring capacity $-10 \%$ & 100 & 0.0000 & 0.0002 & 0.0042 & 2,429 \\
\hline Spring capacity $+10 \%$ & 100 & 0.0000 & 0.0002 & 0.0036 & 2,479 \\
\hline
\end{tabular}


the spring capacity loss fixed at 50 percent, the probability of quasi-extinction doubled to 0.84 percent, and the EMP dropped to 2,229 (table 7).

\section{Behavioral Distribution Rules}

There were no discernible differences among the three behavioral distribution rules used to allocate manatees to the various quality classes of warm-water habitat in their effects on the EMP or the risk of quasi-extinction (table 8).

\section{Discussion}

The manatee Core Biological Model forecasts the population dynamics of the Florida manatee in four regions, integrating current information about life history, incorporating uncertainty in parameter estimates, and applying both environmental and demographic stochasticity. The results in this report represent a comprehensive update to the CBM and provide a framework to assess the current status of manatees, understand the relative effects of the threats manatees face, evaluate concerns around potentially emerging threats, and respond to critiques of past versions of the CBM.

\section{Baseline Status}

The baseline scenario represents our collective judgment about the threats manatees will face over the next century, given the best available scientific and commercial information. The results of the baseline scenario do not represent a continuation of the dynamics of the past several decades; that is, the results are not simply a forecast based on historical conditions. Rather, the baseline scenario incorporated each of the factors affecting manatee population dynamics and included estimates of how those factors will change over time. In some cases, the historical estimates form the basis for the forecast, such as with the baseline annual survival and reproductive rates and their temporal variance. The baseline scenario does assume that current protection and mitigation programs (for example, boat speed zones, rescue and rehabilitation of injured manatees) remain in place indefinitely. The frequency and magnitude of cold events are expected to remain the same, but for most other factors, change over time is expected. Most notably, carrying capacity associated with warm-water habitat is expected to decline, especially over the next half century (2011-60) as power plants with their warm-water effluent are retired and spring flows are reduced. Red-tide events that affect manatees are expected to increase in frequency and possibly severity. The manatee population size is expected to increase; when the population approaches the carrying capacity, we expect more frequent density-dependent cold-related mortality events, as well as declining reproduction.

For evaluating the status of Florida manatees under the ESA, we continue to support the use of a quasi-extinction metric (Runge and others, 2007b); in particular, we propose that the most appropriate single metric is the probability of the population falling below 500 adults on either the Gulf or the East coast over 100 years. First, an extinction risk metric follows directly from the statutory language for classification; an endangered species is one which is "in danger of extinction throughout all or a significant portion of its range" (16 U.S.C. $\$ 1532(6))$. Second, manatees on the two coasts of Florida show weak but statistically significant genetic differentiation (Tucker and others, 2012), and each population represents roughly one-half of the current population size. This suggests to us that loss of one of the coastal populations might constitute extinction in a "significant portion of the range," supporting use of a metric that considers loss on either coast. Third, a combination of arguments leads us to propose 500 adults as a relevant quasi-extinction threshold. In 2007, we argued that an effective population size of 250 was a relevant threshold and equated effective population size with adult breeding

Table 8. Sensitivity of probability of Florida manatee quasi-extinction and expected minimum population size to uncertainty about the behavioral rules used by manatees to distribute among warm-water sites on the Gulf coast or East coast of Florida.

[The values shown are the probability of the adult population on either the Gulf coast or the East coast falling below the specified threshold at any point in the first 100 years of the simulation. In the scenarios for this sensitivity analysis, different behavioral rules for distributing manatees among warm-water sites are considered. The baseline uses a weighted average of the three rules. EMP, expected minimum population size]

\begin{tabular}{|c|c|c|c|c|c|}
\hline Scenario & Years & \multicolumn{3}{|c|}{ Quasi-extinction threshold } & EMP \\
\hline Thermal quality selection & 100 & 0.0000 & 0.0004 & 0.0048 & 2,367 \\
\hline Historical & 100 & 0.0000 & 0.0004 & 0.0046 & 2,360 \\
\hline
\end{tabular}


population size (Runge and others, 2007b). Subsequent genetic work indicates that the ratio of the effective population size to the adult population size in Florida manatees is approximately 0.5 (Tucker and others, 2012); doubling the threshold to 500 adults accounts for this effect. Finally, there appears to be a growing consensus that the objectives of the ESA relate to outcomes on a time scale approaching a century, particularly for large mammals (DeMaster and others, 2004; Regan and others, 2013).

Our estimate of the probability of the Florida manatee population size falling below 500 adults on either the Gulf or the East coast within 100 years is 0.42 percent (table 4 ). This is the same as the estimate from CBMv5 (Runge and others, 2015), but it's considerably lower than the estimate of 49.3 percent from CBMv3 (Runge and others, 2007b). Much has changed in the CBMs between versions 3 and 6; three factors contribute the most to the differences. First, the estimates of adult survival are now higher and more precise owing to substantial improvements in the MIPS database and the methods used to analyze survival (Langtimm and others, 2016). Second, the estimates of carrying capacity are considerably higher than they were in CBMv3 as a result of a carefully structured expert judgment process (M.C. Runge, USGS and C.J. Deutsch, FWRI, written commun., 2017) informed by empirical estimates of capacity at selected warm-water sites (Provancha and others, 2012). Third, the initial population size estimates are higher now than in 2007 as a result of increases in the population size and improved methods for estimating abundance (Martin and others, 2015). Thus, the reduction in the estimate of the risk of quasi-extinction is primarily a reflection of increased understanding of the status of the population rather than an actual improvement in the status of the population, although there are some elements of the latter.

As in previous versions of the CBM, the results forecast a substantial change in the distribution of manatees among regions with long-term declines in the SW and AT regions and substantial long-term increases in the NW and USJ regions (fig. 10). This distributional shift was largely driven by the loss of industrial warm water (fig. 7), a result predicted in a qualitative manner by Laist and Reynolds (2005b). Thus, the coastal metrics of quasi-extinction or EMP mask higher regional risks of decline. These risks are particularly notable for the two largest management units, where probabilities of 30 -percent and 50-percent declines over 100 years are relatively high in the AT (about 0.50 and 0.37 , respectively) and the SW (about 0.46 and 0.29 , respectively) (table 5).

The spatial scale at which assessment is relevant is a policy determination, not a scientific determination, and may differ among policy contexts. The Federal recovery plan for the Florida manatee included a set of population benchmarks that had to be met in each of the four regions prior to reclassification (U.S. Fish and Wildlife Service, 2001); since 2001, the focus has shifted to the coastal, statewide, or species level (U.S. Fish and Wildlife Service, 2007, 2016). For this study, results are reported at the regional, coastal, and subspecies scales, but it is up to policy makers to determine which of those is most appropriate for a particular policy or management decision.

The CBM generates metrics of projected population change that can be applied to IUCN Red List criteria for level of endangerment (IUCN, 2012). Evaluation of manatee status against these criteria is beyond the scope of this study, but estimates of the probability of future population reduction (Criterion A) are worth reporting here. The CBM predicts a statewide reduction in the adult population over three generations (60 years) of greater than or equal to $(\geq) 10$ percent with probability 0.015 ; $\geq 30$ percent with probability 0.003 ; and $\geq 50$ percent with probability $<0.001$. These probabilities are slightly higher over a 100-year time period (table 5). More notably, the chances of decline on either coast over 100 years are substantially higher: $\geq 10$ percent with probability about $0.28, \geq 30$ percent with probability about 0.16 , and $\geq 50$ percent with probability about 0.05 (table 5). We estimate that the risk of major population decline is very low at the statewide (subspecies) level, moderate at the coastal level, and high at the regional level for the two currently largest management units. This seeming paradox stems from the fact that one regional population is expected to decline and one to increase on each coast (fig. 10).

\section{Analysis of Threats}

In previous analyses, watercraft-related mortality and loss of warm-water habitat were the first- and second-ranked threats (Runge and others, 2007b; Runge and others, 2015), as measured by the degree to which removal of the threats reduce the risk of quasi-extinction. In this update, we found that the threat owing to red-tide mortality is now ranked first, as measured by the risk of quasi-extinction, although watercraft and warm-water loss retain their primacy as measured by the EMP (table 4, fig. 13). The strength of red tide as a threat has changed because results of a new expert judgment process predict that red-tide mortality events will become more frequent in the future and that a separate class of red-tide event (intense red tide) may be appearing more often (Julien Martin, USGS, written commun., 2017).

Although the threats presented by red tide, watercraft, and loss of warm water are all relatively strong, they are not absolutely strong because the baseline probability of quasiextinction is low. The situation changes, however, when the possibility of an increase in those threats is considered. In particular, if the rate of watercraft-related mortality were to double, the risk of quasi-extinction would increase more than 10 fold (fig. 14). Because red-tide mortality acts by a mechanism similar to that of watercraft-related mortality, a doubling of the red-tide mortality rate is expected to have a similarly strong effect. Acceleration of loss of capacity at warm-water sites did not show a similarly strong effect on the risk of quasi-extinction (fig. 15) because the warm-water-loss threat acts through a different mechanism (carrying capacity) than watercraft and red tide (mortality), affecting the buffering of 
the population dynamics but not the resilience (Runge and others, 2007b). The cautionary take-home point is that, although the absolute strength of the current threats is low, increases in those threats, especially the ones that affect adult survival (and hence, resilience), could be of concern.

One noteworthy assumption incorporated into the CBM is that manatees using warm water from a power plant that shuts down will be able to find a warm-water refuge of like type (that is, medium-quality industrial) within that region as long as the remaining carrying capacity exceeds the number of manatees using that type of warm-water habitat. In some cases, that is a reasonable assumption; in other cases (for example, an unmitigated loss of warm-water from one of the larger power plants), it is unlikely that all manatees would migrate to warm-water habitat within the region, likely resulting in additional cold-related mortality that is not captured in the model (Laist and Reynolds, 2005b). The CBM, however, was not designed to model such transient effects on mortality (although it does capture the permanent effect on carrying capacity); as long as population growth remains positive, the population should be able to rebound from these mortality events over the long term.

\section{Implications of Recent Cold Mortality Events}

As yet, a full understanding of the cold-mortality events of 2009-10 and 2010-11 is lacking, in part because the annual survival rates for those years are not yet estimable in all regions. But even when those estimates are available, the difficulty of forecasting what they mean for the future remains. An evaluation of four hypotheses about what those events may indicate for the future produced only one explanation with alarming implications. If the reason the number of coldrelated mortalities was so high in those two winters is that the manatee populations have reached their carrying capacities and animals were forced out of good warm-water habitat during a severely cold winter, then the carrying capacities are much lower than we think, and the risk of quasi-extinction is correspondingly higher. This hypothesis flies in the face of the judgment by the expert panel that considered regional availability of warm-water habitat (M.C. Runge, USGS and C.J. Deutsch, FWRI, written commun., 2017); it also contradicts site-specific assessments of carrying capacity (Provancha and others, 2012). Thus, this hypothesis does not seem plausible enough to consider it part of the baseline, but it needs to be considered a candidate, along with the other three hypotheses, to be examined against new data that arise.

\section{Implications of Recent Red-Tide Mortality Events}

The panel of red-tide experts anticipated that red-tide events causing manatee mortality will become more frequent in the future and supported the idea that a separate class of intense red-tide events is possible (Julien Martin, USGS, written commun., 2017). This implies a pessimistic interpretation of the 2013 red-tide mortality in the SW-that it is a harbinger of both more intense and more frequent events in the future. On the basis of the judgment of the expert panel, these effects were included in the baseline scenario of CBMv6 while also accounting for a large degree of uncertainty. The effect of the increased frequency and intensity of red-tide mortality is most pronounced in the SW region (fig. 18) where it acts in concert with the decreasing carrying capacity (fig. 7) to raise the possibility of regional quasi-extinction. The effect of this risk in the SW region, however, is countered by the expected growth of the NW region, so the Gulf coast and statewide probabilities of quasi-extinction remain relatively low (fig. 11). The expert panel did expect some increases in red-tide events in the NW and AT regions, but the effect on those regions is not as strong as for the SW (fig. 18). Taken together, these results indicate that the red-tide mortality may accelerate the change in the population distribution toward the NW and USJ regions but does not pose a major risk of extinction to the subspecies as a whole, unless it spreads more aggressively into the NW and AT regions than the expert panel anticipates.

\section{Public Comments on Previous Assessments}

The Florida manatee population viability assessments conducted in the past with the CBM have been scrutinized carefully by experts and members of the public. Most recently, when the FWS issued a draft rule for reclassifying West Indian manatees from endangered to threatened (U.S. Fish and Wildlife Service, 2016), a public comment period was opened. Some of the public comments addressed the results from CBMv5 (Runge and others, 2015). Here, we respond to some of those comments.

Current threats remain constant. A number of observers raised concern that the CBM assumed "that the various threats operate at their current levels indefinitely" (Runge and others, 2015 , p. 20). We were imprecise in our statement at the time; CBMv5 did include loss of warm-water habitat over time, as well as an increase in cold-related mortality as populations reached their carrying capacities. To address these dynamics, this update (CBMv6) adds to the baseline changes in red-tide frequency and intensity in the periods $0-40$ and $41-100$ years from the start of the simulations (2011). In addition, we have explored scenarios in which watercraft-related mortality increases, industrial warm-water loss is accelerated, carrying capacity at springs is reduced more than anticipated, the frequency of cold increases, and chronic mortality emerges in the AT region. We have offered our assessments of the likelihood of these events in our choice of the baseline scenario, but the full results are available for the reader to examine. We do make an important assumption, however, that existing protections and regulations (for example, speed restriction zones) will continue in the future.

Some threats are not addressed. A number of readers of the CBMv5 assessment noted that there were other potential threats that were not included, like seagrass loss, algal blooms, 
and other unusual mortality events of unknown cause. It is true that no model can fully represent the complexity of the natural world, so no PVA will be able to incorporate all possible events that a population could experience. We have endeavored to include and examine all the threats that are documented or for which a mechanism has been clearly articulated, and there are a couple of features of the model that cover less specific threats (the catastrophic stochasticity owing to emerging disease, the potential for chronic mortality). Threats that fall in the category of "unknown unknowns" are difficult to include, however, as they are, by their nature, unanticipated.

Omission of sublethal and synergistic effects. One detailed comment on the proposed rule raised concern about the omission of sublethal effects in the CBM and the possibility that multiple stressors could interact to produce synergistic (and negative) consequences. To the extent that those dynamics are already acting, they are embedded within the estimates of survival and reproduction and are implicitly included in the model. If, however, the implication is that sublethal effects have not yet reached the point where they are affecting survival and reproductive rates but will do so in the future, we acknowledge the CBM does not yet take that possibility into account, except through the increases in frequency of red tide and cold. The difficulty is postulating a specific mechanism by which the sublethal or synergistic effects could act on life-history parameters, then estimating those effects. Several studies have shown sublethal effects of cold and red-tide exposure on manatee immune function (Walsh and others, 2005, 2015; Sherwood and others, 2015), but the connection between the immune response and subsequent survival or reproductive effects is not yet known. We could postulate mechanisms to include in a model (for instance, reduced survival or reproductive rates in the year following a major red-tide event or interactive effects between red-tide and cold-related mortality) but have not yet included such mechanisms in the CBM.

\section{Core Biological Model Developments}

This comprehensive revision of the CBM added a number of detailed dynamics that previous versions had been missing, bringing it to a level of functionality and flexibility that allows for a host of questions to be addressed. As a result, the promising areas of future development revolve around generating hypotheses from the model that can be examined in nature to answer fundamental questions about manatee population ecology.

Density-dependent population regulation. The CBM contains two mechanisms for regulating population growth: density-dependent mortality associated with cold events, as affected by the distribution of animals among warm-water sites of different quality, and density-dependent reduction in reproductive rates. In a series of error-checking scenarios conducted during the development of CBMv6, we discovered that the density-dependent mortality mechanism alone (as implemented in the model) is not enough to regulate the population, but the density-dependent reproduction mechanism is. This was somewhat surprising because the common wisdom is that warm-water habitat, through exposure to cold mortality, is the limiting resource for Florida manatees. This finding of insufficient density-dependent mortality may stem from bias in the estimates of additional cold mortality in low-quality warm-water habitat (from the fractions-of-mortality analysis) because relatively few of the carcasses in this habitat class were from north Florida where water temperatures commonly drop below the manatee's physiological tolerance. If manatees were forced to rely only on low-quality habitat throughout the winter, we would expect cold mortality to be higher than estimated (fig. 6) for manatees in the two northern regions (NW, USJ) and the northern portions of the other two regions (for example, SW north of Sarasota, Fla.; AT north of Sebastian). On the other hand, in long-lived mammals, we might expect the adult survival rates to be fairly robust to environmental forces and the reproductive rates to be the mechanism by which density dependence acts. This is a fruitful area for generating testable hypotheses about density dependence in manatee populations and for designing experiments or monitoring to evaluate these hypotheses.

Sublethal and synergistic effects. As discussed above, the CBM (CBMv6) does not yet have explicit mechanisms for sublethal or synergistic effects. We are interested in exploring demographic mechanisms by which these effects may manifest in survival or reproductive rates and using a future version of the CBM to generate hypotheses that could be tested in the field.

Effects of climate change. The examination of the hypotheses about cold-related mortality left open an interesting question: What do the climate models forecast regarding the frequency and intensity of cold in Florida? Work is underway to couple downscaled climate forecasts with the CBM to explore this question. Beyond the effects of cold, a large set of potential effects of climate change could be examined, including changes in the frequency and severity of algal blooms, changes in the frequency and intensity of storms, sea-level rise (and associated changes to warm-water and foraging habitats), effects on seagrass, and changes in disease dynamics (Edwards, 2013). Discussions about how to incorporate such effects into the CBM have only just begun.

Continued improvements to parameter estimation. Building on the quality and duration of the MIPS and carcass data series, as well as other advances in data collection, new analytical methods for estimating population demographic parameters have been developed for manatee assessment that have been valuable far beyond the manatee world. We expect such work to continue and look forward to continued advances in survival and reproductive estimation, Bayesian hierarchical estimation of mortality fractions, empirical estimation of carrying capacity, and empirical methods for forecasting cold, red tide, and changes in carrying capacity. 


\section{Summary and Conclusions}

This report describes a comprehensive revision and update of the manatee Core Biological Model (CBM), undertaken by the U.S. Geological Survey and the Florida Fish and Wildlife Conservation Commission to evaluate the status of, and severity of threats to, Trichechus manatus latirostris (Florida manatee). This work builds on previous versions of the CBM, extending the capability of the model to incorporate more detailed population dynamics and to address more specific questions about certain threats while updating all of the parameter estimates that underlie the forecasts.

A baseline-and-scenarios approach to evaluation was used, with the baseline scenario representing the best single summary of the available information about the status of the subspecies and the threats it faces over the next century and additional scenarios providing insight about the nature and strength of the threats. Under the baseline scenario, the probability of the Florida manatee population falling below a quasi-extinction level of 500 adults on either the Gulf or East coast of Florida within the next 100 years from 2011 was estimated to be 0.42 percent. This risk is low because the estimated adult survival rates are high $(>0.97)$, the current population size is greater than 2,500 on each coast, and the estimated carrying capacity for manatees is much larger than the current abundance estimates in all four regions of Florida. Expected losses of warm-water habitat are likely to cause a major change in the distribution of the population from the regions where manatees rely heavily on power-plant effluents for warmth in winter (Southwest and Atlantic regions) to the regions where manatees primarily use natural springs in winter (Northwest and Upper St. Johns regions). The probabilities that the manatee populations in the Southwest and Atlantic regions will decrease by at least 30 percent over the next century are 46 percent and 50 percent, respectively. Thus, the relatively low probabilities of quasi-extinction at the coastal scale mask higher risks of decline at the regional level.

A series of scenarios that removed or increased the existing threats was used to evaluate the strength of those threats. Three threats contribute in roughly equal measures to the risk of quasi-extinction: watercraft-related mortality, red-tide mortality, and loss of warm-water habitat. Only an increase in watercraft-related mortality has the potential to substantially increase the risk of dropping below 500 adults on either coast in 100 years, from 0.42 percent under the baseline to nearly 4 percent if the rate of watercraft-related mortality were to double. Accelerated loss of industrial warm-water effluent, on the other hand, does not increase the same risk of quasi-extinction above 1 percent.
One of the goals of this analysis was to explore a series of hypotheses for the recent unprecedented manatee mortality events, including the cold-induced mortality events of 2009-10 and 2010-11 and the red-tide mortality event of 2013. One of the hypotheses about the cold events stands out: if winter carrying capacity has been reached, then a densitydependent mechanism likely underlies the observed coldrelated mortality; this would mean there is less capacity to buffer the population from future threats and the corresponding estimates of quasi-extinction would be nearly 20 -fold higher than baseline. This hypothesis does not align with the results of an expert panel convened to review and estimate manatee carrying capacity, but it could gain more credence if future cold events consistently induce mortality like the 2009-10 and 2010-11 events. Among the red-tide hypotheses, the ones that forecast a future increase in the frequency and magnitude of manatee red-tide mortality were included in the baseline results. The increases in red-tide mortality have the strongest negative effect in the Southwest region, but this effect is balanced by the forecast growth in the Northwest region, so the threat is not large at the coastal level.

A number of other scenarios were examined to explore the possible effects of potential emerging threats, and in most of them, the risk of quasi-extinction at the coastal scale within 100 years did not rise above 1 percent. The three exceptions are scenarios in which carrying capacity is only a fraction of the current estimates, a new chronic source of mortality emerges, or multiple threats emerge in concert. Even in these scenarios, however, the risk of coastal quasi-extinction within 100 years is less than 10 percent using a threshold of 500 adults and less than 1 percent using a threshold of 250 adults.

The long-term data and analytical methods that form the foundation of the CBM have improved over time and, with continued support for population research and monitoring, we expect that evolution to continue. The scientific and management questions that guide the structure of the CBM have likewise evolved since its inception as our knowledge and insights have improved. The CBM will continue to be updated in response to new information and questions. Future developments may include the exploration of density-dependent mechanisms, synergistic effects, and climate-change effects.

High adult survival provides the Florida manatee population with strong resilience to a variety of current and future threats, and a large estimated carrying capacity provides a buffer to short-term reductions in the population from episodic threats. If vigilance in management is reduced, the scenarios in which manatees could face risk of decline become more likely. But on the basis of these analyses, we conclude that, if the threats that manatees face continue to be managed effectively, manatees are likely to persist on both coasts of Florida and remain an integral part of the coastal Florida ecosystem through the 21 st century. 


\section{References Cited}

Barlas, M.E., Deutsch, C.J., de Wit, M., and Ward-Geiger, L.I., 2011, Florida manatee cold-related unusual mortality event, January-April 2010: St. Petersburg, Fla., Florida Fish and Wildlife Conservation Commission, 138 p., http://myfwc. com/media/1536184/2010_Manatee_Cold_related_UME_ Final.pdf.

Bossart, G.D., Meisner, R.A., Rommel, S.A., Ghim, S.-j., and Jenson, A.B., 2002, Pathological features of the Florida manatee cold stress syndrome: Aquatic Mammals, v. 29, no. 1, p. 9-17.

Burgman, M., Carr, A., Godden, L., Gregory, R., McBride, M., Flander, L., and Maguire, L., 2011, Redefining expertise and improving ecological judgment: Conservation Letters, v. 4 , no. 2 , p. 81-87.

Burnham, K.P., Anderson, D.R., White, G.C., Brownie, C., and Pollock, K.H., 1987, Design and analysis methods for fish survival experiments based on release-recapture: American Fisheries Society Monograph, v. 5, p. 1-437.

Castelblanco-Martínez, D.N., Nourisson, C., Quintana-Rizzo, E., Padilla-Saldivar, J., and Schmitter-Soto, J.J., 2012, Potential effects of human pressure and habitat fragmentation on population viability of the Antillean manatee Trichechus manatus manatus - a predictive model: Endangered Species Research, v. 18, no. 2, p. 129-145.

DeMaster, D.P., Angliss, R., Cochrane, J.F., Mace, P., Merrick, R., Miller, M., Rumsey, S., Taylor, B.L., Thompson, G., and Waples, R., 2004, Recommendations to NOAA FisheriesESA listing criteria by the quantitative working group: U.S. Department of Commerce, NOAA Technical Memorandum NMFS-F/SPO-67, 85 p.

Deutsch, C.J., Reid, J.P., Bonde, R.K., Easton, D.E., Kochman, H.I., and O'Shea, T.J., 2003, Seasonal movements, migratory behavior, and site fidelity of West Indian manatees along the Atlantic coast of the United States: Wildlife Monographs, v. 151, p. 1-77.

Deutsch, C.J., Self-Sullivan, C., and Mignucci-Giannoni, A., 2008, Trichechus manatus-The IUCN Red List of threatened species: International Union for Conservation of Nature, v. 2008, no. e.T22103A9355917.

Duignan, P.J., House, C., Walsh, M.T., Campbell, T., Bossart, G.D., Duffy, N., Fernandes, P.J., Rima, B.K., Wright, S., and Geraci, J. R., 1995, Morbillivirus infection in manatees: Marine Mammal Science, v. 11, no. 4, p. 441-451.
Eberhardt, L.L., and O'Shea, T.J., 1995, Integration of manatee life-history data and population modeling, in O'Shea, T.J., Ackerman, B.B., and Percival, H.F., eds., Population biology of the Florida Manatee: Washington, D.C., U.S. Department of the Interior, National Biological Service, p. 269-279.

Edwards, H.H., 2013, Potential impacts of climate change on warmwater megafauna: the Florida manatee example (Trichechus manatus latirostris): Climatic Change, v. 121, no. 4, p. 727-738.

Florida Fish and Wildlife Conservation Commission, 2007, Florida manatee management plan, Trichechus manatus latirostris: Tallahassee, Fla., Florida Fish and Wildlife Conservation Commission, 267 p., http://myfwc.com/ media/415297/manateemgmtplan.pdf.

Florida Marine Research Institute, 2002, Population viability analysis of the Florida manatee (Trichechus manatus latirostris), in Florida Marine Research Institute, ed., Final biological status review of the Florida Manatee (Trichechus manatus latirostris): St. Petersburg, Fla., Florida Fish and Wildlife Conservation Commission, p. 36-72.

Gao, Y., Leung, L.R., Lu, J., and Masato, G., 2015, Persistent cold air outbreaks over North America in a warming climate: Environmental Research Letters, v. 10, no. 4, p. 044001.

Hanea, A., McBride, M., Burgman, M., Wintle, B., Fidler, F., Flander, L., Twardy, C., Manning, B., and Mascaro, S., 2017, I nvestigate D iscuss E stimate A ggregate for structured expert judgement: International Journal of Forecasting, v. 33, no. 1, p. 267-279.

Harwood, J., and Hall, A., 1990, Mass mortality in marine mammals: its implications for population dynamics and genetics: Trends in Ecology and Evolution, v. 5, no. 8, p. $254-257$.

Haubold, E.M., Deutsch, C.J., and Fonnesbeck, C.J., 2006, Final biological status review of the Florida manatee (Trichechus manatus latirostris): St. Petersburg, Fla., Florida Fish and Wildlife Conservation Commission. 63 p., http:// myfwc.com/media/201862/finalreport_3230.pdf.

International Union for Conservation of Nature (IUCN), 2012, IUCN Red List Categories and Criteria-Version 3.1 (2d ed.): Gland, Switzerland, and Cambridge, UK, International Union for Conservation of Nature, iv $+32 \mathrm{p}$.

Kendall, W.L., Barker, R.J., White, G.C., Lindberg, M.S., Langtimm, C.A., and Peñaloza, C.L., 2013, Combining dead recovery, auxiliary observations and robust design data to estimate demographic parameters from marked individuals: Methods in Ecology and Evolution, v. 4, no. 9, p. 828-835. 
Kendall, W.L., Langtimm, C.A., Beck, C.A., and Runge, M.C., 2004, Capture-recapture analysis for estimating manatee reproductive rates: Marine Mammal Science, v. 20, no. 3, p. 424-437.

Kendall, W.L., White, G.C., Hines, J.E., Langtimm, C.A., and Yoshizaki, J., 2012, Estimating parameters of hidden Markov models based on marked individuals - use of robust design data: Ecology, v. 93, no. 4, p. 913-920.

Kullback, S., and Leibler, R.A., 1951, On information and sufficiency: The Annals of Mathematical Statistics, v. 22, no. 1, p. 79-86.

Laist, D.W., and Reynolds, J.E., III, 2005a, Influence of power plants and other warm-water refuges on Florida manatees: Marine Mammal Science, v. 21, no. 4, p. 739-764.

Laist, D.W., and Reynolds, J.E., III, 2005b, Florida manatees, warm-water refuges, and an uncertain future: Coastal Management, v. 33, no. 3, p. 279-295.

Lande, R., 1993, Risks of population extinction from demographic and environmental stochasticity and random catastrophes: The American Naturalist, v. 142, no. 6, p. 911-927.

Landsberg, J.H., Flewelling, L.J., and Naar, J.P., 2009, Karenia brevis red tides, brevetoxins in the food web, and impacts on natural resources-Decadal advancements: Harmful Algae, v. 8, no. 4, p. 598-607.

Langtimm, C.A., 2009, Non-random temporary emigration and the robust design - Conditions for bias at the end of a time series, in Thomson, D.L., Cooch, E.G., and Conroy, M.J., eds., Modeling demographic processes in marked populations: Boston, Mass., USA, Springer, p. 745-761.

Langtimm, C.A., Beck, C.A., Edwards, H.H., Fick-Child, K.J., Ackerman, B.B., Barton, S.L., and Hartley, W.C., 2004, Survival estimates for Florida manatees from the photoidentification of individuals: Marine Mammal Science, v. 20 , no. 3 , p. $438-463$.

Langtimm, C.A., Kendall, W.L., Beck, C.A., Kochman, H.I., Teague, A.L., Meigs-Friend, G., and Peñaloza, C.L., 2016, Model description and evaluation of the mark-recapture survival model used to parameterize the 2012 status and threats analysis for the Florida manatee (Trichechus manatus latirostris): U.S. Geological Survey Open-File Report 2016-1163, 20 p., http://dx.doi.org/10.3133/ofr20161163.

Lefebvre, L.W., Marmontel, M., Reid, J.P., Rathbun, G.B., and Domning, D.P., 2001, Status and biogeography of the West Indian manatee, in Woods, C.A., and Sergile, F.E., eds., Biogeography of the West Indies ( $2 \mathrm{~d}$ ed.): Boca Raton, Fla., CRC Press, p. 425-474.
Lipscomb, T.P., Schulman, F.Y., Moffett, D., and Kennedy, S., 1994, Morbilliviral disease in Atlantic bottlenose dolphins (Tursiops truncatus) from the 1987-1988 epizootic: Journal of Wildlife Diseases, v. 30, no. 4, p. 567-571.

Lunn, D.J., Thomas, A., Best, N., and Spiegelhalter, D., 2000, WinBUGS - a Bayesian modelling framework-concepts, structure, and extensibility: Statistics and Computing, v. 10, no. 4 , p. 325-337.

Marmontel, M., Humphrey, S.R., and O'Shea, T.J., 1997, Population viability analysis of the Florida manatee (Trichechus manatus latirostris), 1976-1991: Conservation Biology, v. 11 , no. 2 , p. $467-481$.

Martin, J., Edwards, H.H., Fonnesbeck, C.J., Koslovsky, S.M., Harmak, C.W., and Dane, T.M., 2015, Combining information for monitoring at large spatial scales-First statewide abundance estimate of the Florida manatee: Biological Conservation, v. 186, p. 44-51.

McCarthy, M.A., and Thompson, C.J., 2001, Expected minimum population size as a measure of threat: Animal Conservation, v. 4, p. 351-355.

McGowan, C.P., Runge, M.C., and Larson, M.A., 2011, Incorporating parametric uncertainty into population viability analysis models: Biological Conservation, v. 144, p. 1400 1408 .

Morris, W.F., and Doak, D.F., 2002, Quantitative conservation biology - theory and practice of population viability analysis: Sunderland, Mass., Sinauer Associates, 480 p.

NIMBLE Development Team, 2016, NIMBLE-an R package for programming with BUGS models, accessed July 6 , 2016, at http://r-nimble.org.

Packard, J.M., 1985, Preliminary assessment of uncertainty involved in modeling manatee populations: Florida Cooperative Fish and Wildlife Research Unit Manatee Population Research Report No. 9, 19 p.

Peñaloza, C.L., Kendall, W.L., and Langtimm, C.A., 2014, Reducing bias in survival under nonrandom temporary emigration: Ecological Applications, v. 24, no. 5, p. 1155-1166.

Phlips, E.J., Badylak, S., Lasi, M.A., Chamberlain, R., Green, W.C., Hall, L.M., Hart, J.A., Lockwood, J.C., Miller, J.D., and Morris, L.J., 2015, From red tides to green and brown tides - bloom dynamics in a restricted subtropical lagoon under shifting climatic conditions: Estuaries and Coasts, v. 38 , no. 3, p. 886-904.

Provancha, J., Taylor, C., Gimond, M., Wild, M., and Rouhani, S., 2012, K-assessment of manatee forage and warm-water associated with eleven Florida sites: Merritt Island, Fla., Innovative Health Applications, LLC, 123 p. 
R Development Core Team, 2015, R - a language and environment for statistical computing: Vienna, Austria, R Foundation for Statistical Computing, accessed March 24, 2017, at http://www.R-project.org/.

Rayer, S., and Wang, Y., 2016, Projections of Florida population by county, 2020-2045, with estimates for 2015: Florida Population Studies, v. 49, no. 174, p. 1-8.

Regan, T.J., Taylor, B.L., Thompson, G.G., Cochrane, J.F., Ralls, K., Runge, M.C., and Merrick, R., 2013, Testing decision rules for categorizing species' extinction risk to help develop quantitative listing criteria for the U.S. Endangered Species Act: Conservation Biology, v. 27, no. 4, p. 821-831.

Reid, J., Rathbun, G.B., and Wilcox, J.R., 1991, Distribution patterns of individually identifiable West Indian manatees (Trichechus manatus) in Florida: Marine Mammal Science, v. 7, no. 2, p. 180-190.

Rodenhouse, N.L., Sherry, T.W., and Holmes, R.T., 1997, Sitedependent regulation of population size - a new synthesis: Ecology, v. 78, no. 7, p. 2025-2042.

Rouhani, S., Sucsy, P., Hall, G., Osburn, W., and Wild, M., 2007, Analysis of Blue Spring discharge data to determine a minimum flow regime: St. Johns River Water Management District Special Publication, v. SJ2007-SP17, 52 p. + appendices.

Runge, M.C., 2003, A model for assessing incidental take of manatees due to watercraft-related activities, in U.S. Fish and Wildlife Service, ed., Environmental Impact Statement: Rulemaking for the incidental take of small numbers of Florida manatees (Trichechus manatus latirostris) resulting from government programs related to watercraft access and watercraft operation in the state of Florida: Jacksonville, Fla., U.S. Fish and Wildlife Service, p. Appendix I.

Runge, M.C., Langtimm, C.A., and Kendall, W.L., 2004, A stage-based model of manatee population dynamics: Marine Mammal Science, v. 20, no. 3, p. 361-385.

Runge, M.C., Langtimm, C.A., Martin, J., and Fonnesbeck, C.J., 2015, Status and threats analysis for the Florida manatee (Trichechus manatus latirostris), 2012: U.S. Geological Survey Open-File Report 2015-1083, 23 p., http://dx.doi. org/10.3133/ofr20151083.

Runge, M.C., Sanders-Reed, C.A., and Fonnesbeck, C.J., 2007a, A core stochastic population projection model for Florida manatees (Trichechus manatus latirostris): U.S. Geological Survey Open-File Report 2007-1082, 41 p., https://pubs.er.usgs.gov/publication/ofr20071082.
Runge, M.C., Sanders-Reed, C.A., Langtimm, C.A., and Fonnesbeck, C.J., 2007b, A quantitative threats analysis for the Florida manatee (Trichechus manatus latirostris): U.S. Geological Survey Open-File Report 2007-1086, 34 p., https:// pubs.er.usgs.gov/publication/ofr20071086.

Schlaepfer, M.A., Runge, M.C., and Sherman, P.W., 2002, Ecological and evolutionary traps: Trends in Ecology \& Evolution, v. 17, no. 10, p. 474-480.

Sherwood, T.A., Reynolds, J.E., III, and Wetzel, D.L., 2015, Immune assessment in manatees with cold stress syndrome [abs.]: Fifth Florida Marine Mammal Health Conference, June 2-4, 2015, Gainesville, Florida, p. 58.

Speirs-Bridge, A., Fidler, F., McBride, M.F., Flander, L., Cumming, G., and Burgman, M.A., 2010, Reducing overconfidence in the interval judgments of experts: Risk Analysis, v. 30 , no. 3 , p. $512-523$.

Tucker, K.P., Hunter, M.E., Bonde, R.K., Austin, J.D., Clark, A.M., Beck, C.A., McGuire, P.M., and Oli, M.K., 2012, Low genetic diversity and minimal population substructure in the endangered Florida manatee-implications for conservation: Journal of Mammalogy, v. 93, no. 6, p. 1504-1511.

U.S. Fish and Wildlife Service, 2001, Florida manatee recovery plan, (Trichechus manatus latirostris), third revision: Atlanta, Ga., U.S. Fish and Wildlife Service, 144 p.

U.S. Fish and Wildlife Service, 2007, West Indian Manatee (Trichechus manatus) 5-year Review: Summary and Evaluation: Jacksonville, Fla., U.S. Fish and Wildlife Service, Jacksonville Ecological Services Office, 79 p.

U.S. Fish and Wildlife Service, 2016, Endangered and threatened wildlife and plants; 12-month finding on a petition to downlist the West Indian manatee, and proposed rule to reclassify the West Indian manatee as threatened: Federal Register, v. 81, no. 5, p. 1000-1026.

Walsh, C.J., Butawan, M., Yordy, J., Ball, R., Flewelling, L., de Wit, M., and Bonde, R.K., 2015, Sublethal red tide toxin exposure in free-ranging manatees (Trichechus manatus) affects the immune system through reduced lymphocyte proliferation responses, inflammation, and oxidative stress: Aquatic Toxicology, v. 161, p. 73-84.

Walsh, C.J., Luer, C.A., and Noyes, D.R., 2005, Effects of environmental stressors on lymphocyte proliferation in Florida manatees, Trichechus manatus latirostris: Veterinary immunology and immunopathology, v. 103, no. 3, p. 247-256.

White, G.C., and Burnham, K.P., 1999, Program MARK: survival estimation from populations of marked animals: Bird study, v. 46, no. S1, p. S120-S139. 
Publishing support provided by the

U.S. Geological Survey Science Publishing Network, West Trenton Publishing Service Center

For more information concerning the research in this report, contact: Director, Patuxent Wildlife Research Center

U.S. Geological Survey

12100 Beech Forest Road, Ste 4039

Laurel, MD 20708-4039

http://www.pwrc.usgs.gov/ 


\section{$\frac{\mathbb{2}}{\mathrm{C}}$}
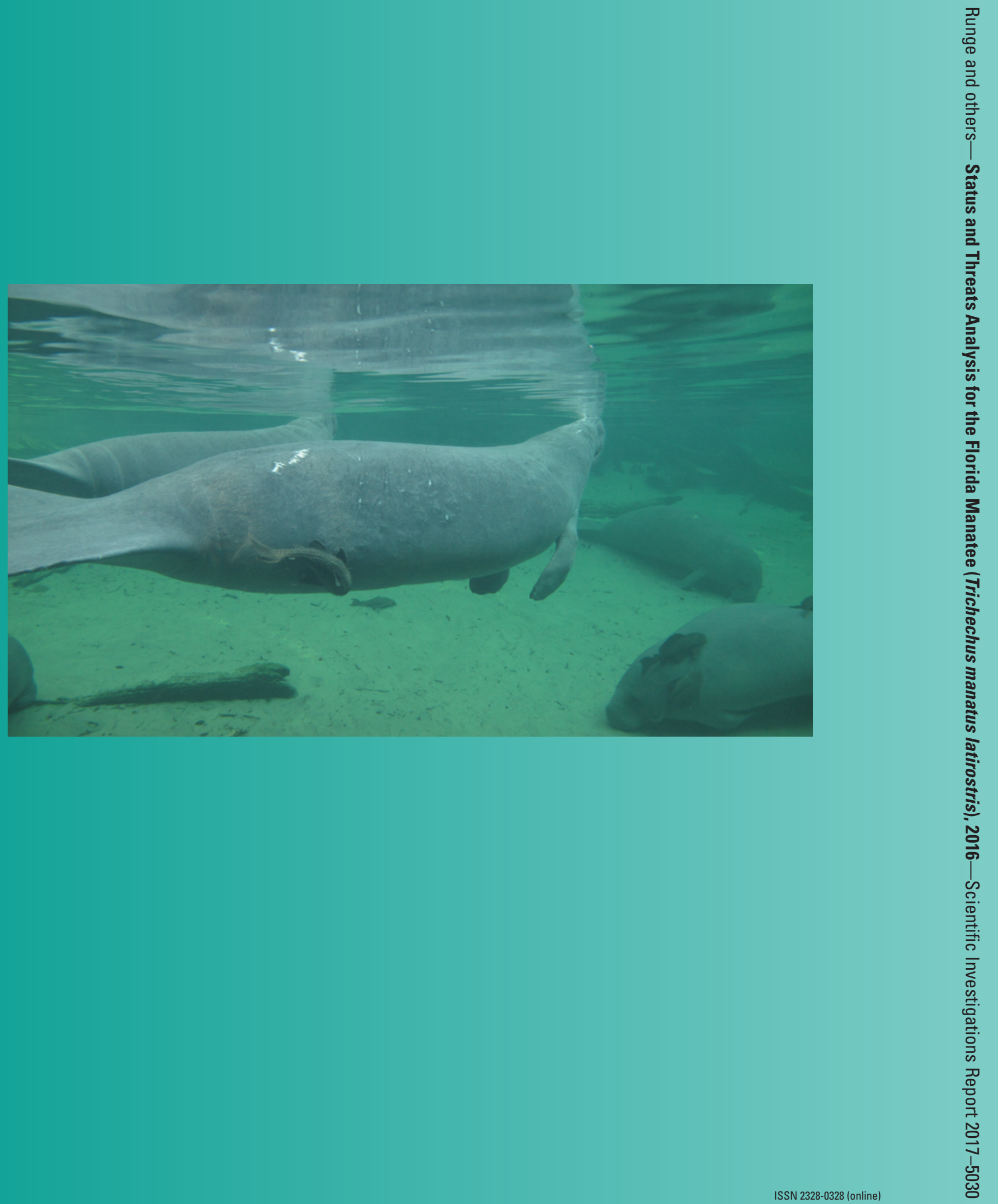